Portland State University

PDXScholar

$1-1-2012$

\title{
Reconceptualizing Organizational Commitment Using the Theory of Reasoned Action: Testing Antecedents of Multiple Organizational Behaviors
}

Thu Gia Hoang

Portland State University

Follow this and additional works at: https://pdxscholar.library.pdx.edu/open_access_etds Let us know how access to this document benefits you.

Recommended Citation

Hoang, Thu Gia, "Reconceptualizing Organizational Commitment Using the Theory of Reasoned Action: Testing Antecedents of Multiple Organizational Behaviors" (2012). Dissertations and Theses. Paper 109. https://doi.org/10.15760/etd.109

This Dissertation is brought to you for free and open access. It has been accepted for inclusion in Dissertations and Theses by an authorized administrator of PDXScholar. Please contact us if we can make this document more accessible: pdxscholar@pdx.edu. 
Reconceptualizing Organizational Commitment Using the Theory of Reasoned Action:

Testing Antecedents of Multiple Organizational Behaviors

by

Thu Gia Hoang

A dissertation submitted in partial fulfillment of the requirements for the degree of

Doctor of Philosophy

in

Applied Psychology

Dissertation Committee:

Keith James, Chair

John L. Gallup

Marcus D. Ingle

Dalton Miller-Jones

Mo Wang

Portland State University

(C)2012 


\begin{abstract}
The Three-Component Model of organizational commitment (TCM) by Meyer and Allen $(1991,1997)$ is widely regarded as the most dominant model in organizational commitment research (Cohen, 2003, 2007). However, recent research by Solinger et al. (2008) questioned the legitimacy of the TCM as a general model of organizational commitment. More specifically, the authors criticized the TCM for grouping affective commitment as an attitude toward target with continuance and normative commitment as attitudes toward behaviors under one general label of attitudinal construct. Based on the Theory of Reasoned Action (TRA; Ajzen \& Fishbein, 1980), Solinger et al. (2008) argued that we should consider organizational commitment strictly as an attitude toward the organization (i.e., affective commitment).

Based on Eagly and Chaiken's (1993) composite attitude-behavior model, the current study tested the reconceptualization of organizational commitment as a unidimensional construct reflecting employees' attitudes toward the organization (i.e., affective commitment) in predicting several organizational behaviors (i.e., considerate voice, production deviance, and behavioral engagement). In addition, I also investigated whether these organizational behaviors could be better explained by adding different classes of behavioral expectancies (i.e., utilitarian, normative and self-identity expectancies) as antecedents. Finally, I tested the mediating roles of attitude toward behaviors in the relationship between affective commitment and three behavioral expectancies and the three organizational behaviors. A sample of 258 employees in a large-sized organization in China was obtained for this study. The results suggested that
\end{abstract}


none of the hypotheses of the current study was supported by the evidence in the current study. In particular, affective commitment and three classes of behavioral expectancies did not significantly predict their corresponding behaviors. In addition, I also did not find the evidence for the indirect effects from affective commitment and the behavioral expectancies on the behaviors.

Several alternative explanations were provided for the results. Among those, the lack of compatibility between affective commitment and the behaviors, the existence of moderators (e.g. national culture), the lack of necessary control to perform the behaviors successfully are key factors that might lead to the current findings. Although none of the hypotheses was supported, I found limited empirical supports for the reconceptualization of organizational commitment strictly as the attitude toward the organization and that organizational behaviors could be better explained by adding appropriate behavioral expectancies to the model (Solinger et al., 2008). Finally, theoretical and practical implications of the current study as well as directions for future research are discussed. 


\section{Dedication}

I dedicate my dissertation work to my family. I would like to extend a special gratitude and thank my parents, Hoàng Văn Bắc and Nguyễn Thị Trọng, for their unwavering support, love, and wisdoms. I thank my brother, Bá, for his caring and for his support of the family while I am away from home. I dedicate this work and give a special thanks to my dear wife, Quỳnh Anh, for her sacrifice, love and support throughout the entire doctorate program. Last but not least, I dedicate this dissertation to my wonderful son, Minh, who always put a smile on my face whenever I think of him. 


\section{Acknowledgements}

I would like to acknowledge and thank my former advisor, Dr. Mo Wang, for his guidance and continuing support throughout the entire program, even after moving to other institutions. I also would like to acknowledge and thank my advisor, Dr. Keith James, for his support and guidance, even when he was on leave to serve as a program officer at the National Science Foundation. Additionally, I would like to thank my dissertation committee for their time and the feedback they have provided. Finally, I would like to acknowledge and thank Dr. Junqi Shi at Peking University for the data collection. Without their help, this dissertation would not have been possible. 
Table of Contents

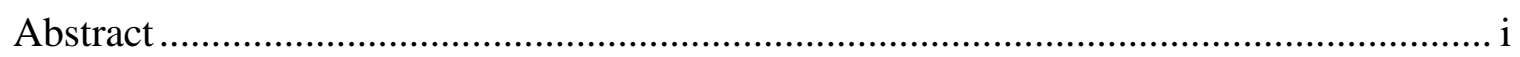

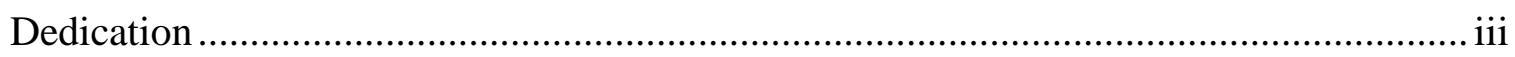

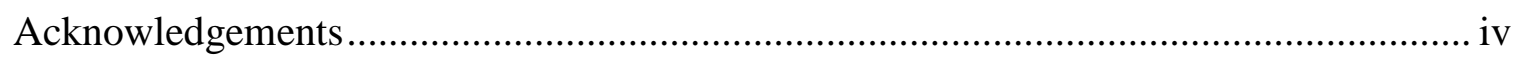

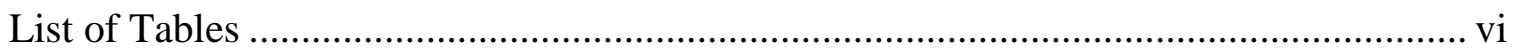

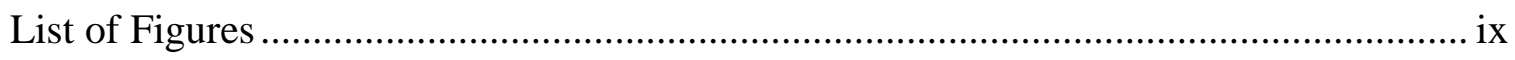

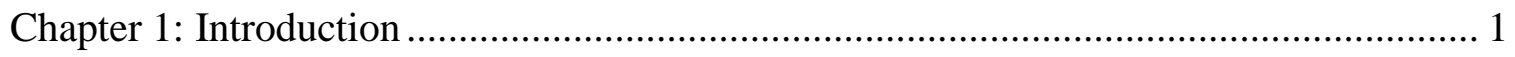

Chapter 2: The three-component model of organizational commitment .......................... 5

Chapter 3: Theory of reasoned action and conceptualization of organizational

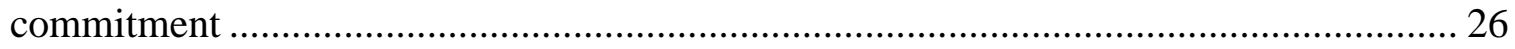

Chapter 4: Organizational behavior criteria and hypothesis development ..................... 33

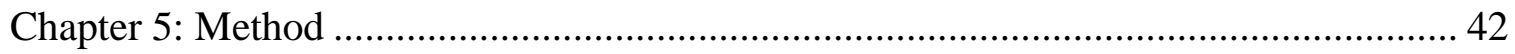

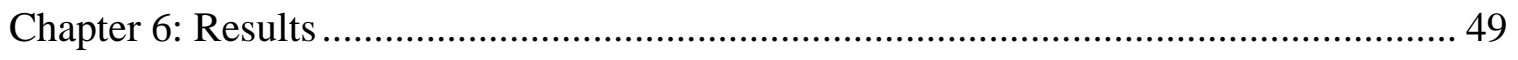

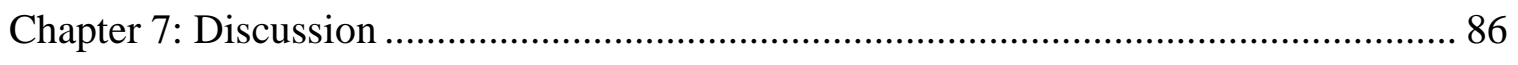

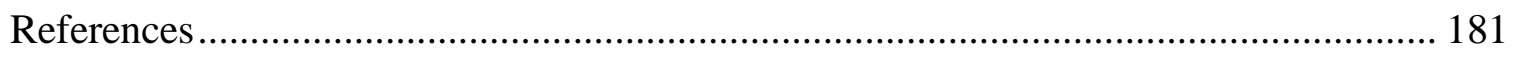

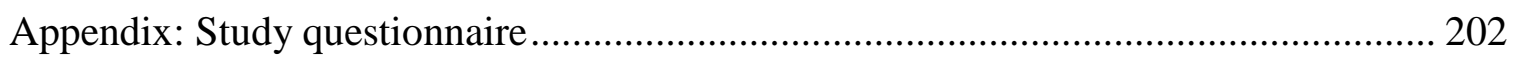


List of Tables

Table 1. Inter-Item Correlations for Utilitarian Expectancies of Considerate Voice ..... 126

Table 2. Exploratory Factor Analysis for Utilitarian Expectancies of Considerate Voice

Table 3. Inter-Item Correlations for Normative Expectancies of Considerate Voice..... 128

Table 4. Exploratory Factor Analysis for Normative Expectancies of Considerate Voice

Table 5. Inter-Item Correlations for Self-Identity Expectancies of Considerate Voice . 130

Table 6. Exploratory Factor Analysis for Self-Identity Expectancies of Considerate Voice 131

Table 7. Inter-Item Correlations for Attitude toward Considerate Voice...................... 132

Table 8. Exploratory Factor Analysis for Attitude toward Considerate Voice............... 134

Table 9. Inter-Item Correlations for Considerate Voice ............................................ 135

Table 10. Exploratory Factor Analysis for Considerate Voice .................................. 136

Table 11. Confirmatory Factor Analysis Results for Scales involved Considerate Voice 137

Table 12. Exploratory Factor Analysis Results for the Final Scales involved Considerate

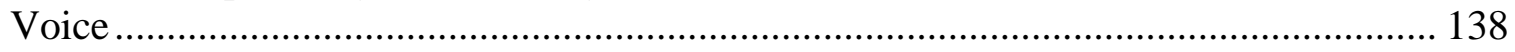

Table 13. Inter-Item Correlations for Utilitarian Expectancies of Production Deviance 141

Table 14. Exploratory Factor Analysis for Utilitarian Expectancies of Production

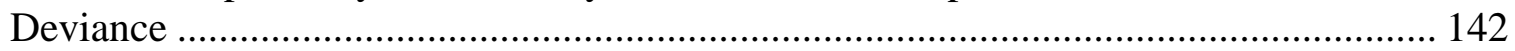

Table 15. Inter-Item Correlations for Normative Expectancies of Production Deviance143

Table 16. Exploratory Factor Analysis for Normative Expectancies of Production

Deviance

Table 17. Inter-Item Correlations for Self-Identity Expectancies of Production Deviance 
Table 18. Exploratory Factor Analysis for Self-Identity Expectancies of Production

Deviance 146

Table 19. Inter-Item Correlations for Attitude toward Production Deviance 147

Table 20. Exploratory Factor Analysis for Attitude toward Production Deviance 148

Table 21. Inter-Item Correlations for Production Deviance 149

Table 22. Exploratory Factor Analysis for Production Deviance 150

Table 23. Confirmatory Factor Analysis Results for Scales involved Production Deviance 151

Table 24. Exploratory Factor Analysis for the Final Scales involved Production Deviance 152

Table 25. Inter-Item Correlations for Utilitarian Expectancies of Behavioral Engagement

Table 26. Exploratory Factor Analysis for Utilitarian Expectancies of Behavioral Engagement. 154

Table 27. Inter-Item Correlations for Normative Expectancies of Behavioral Engagement 155

Table 28. Exploratory Factor Analysis for Normative Expectancies of Behavioral Engagement. 156

Table 29. Inter-Item Correlations for Self-Identity Expectancies of Behavioral Engagement. 157

Table 30. Exploratory Factor Analysis for Self-Identity Expectancies of Behavioral Engagement. 158

Table 31. Inter-Item Correlations for Attitude toward Behavioral Engagement 159

Table 32. Exploratory Factor Analysis for Attitude toward Behavioral Engagement.... 161

Table 33. Inter-Item Correlations for Behavioral Engagement 162

Table 34. Exploratory Factor Analysis for Behavioral Engagement 163 
Table 35. Confirmatory Factor Analysis Results for Scales involved Behavioral Engagement

Table 36. Exploratory Factor Analysis for the Final Scales involved Behavioral

Engagement. 165

Table 37. Confirmatory Factor Analysis Results for the Hypothesized Models 166

Table 38. Exploratory Factor Analysis for Organizational Commitment Scale 167

Table 39. Exploratory Factor Analysis for Affective Commitment, Continuance

Commitment, and Normative Commitment 168

Table 40. Means, Standard Deviations, and Correlations among Study Variables 169

Table 41. Standardized Structural Path Coefficients for Study Variables on Mediator and Considerate Voice in the Full Mediation Model (M1) .....

Table 42. Standardized Structural Path Coefficients for Study Variables on Mediator and Production Deviance in the Full Mediation Model (M1) 172

Table 43. Standardized Structural Path Coefficients for Study Variables on Mediator and Behavioral Engagement in the Full Mediation Model (M1) 173

Table 44. Bootstrap Confidence Intervals for the Indirect Effects 174 


\section{List of Figures}

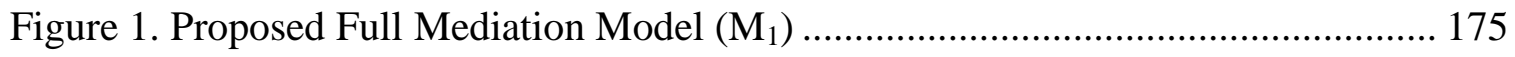

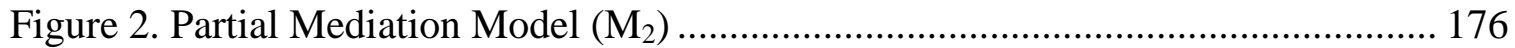

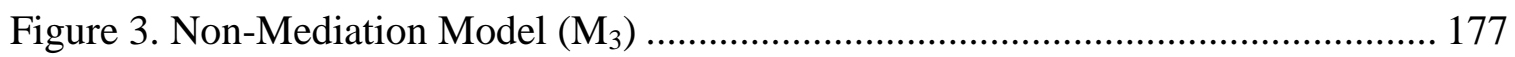

Figure 4. Full Mediation Model $\left(\mathrm{M}_{1}\right)$ for Considerate Voice ..................................... 178

Figure 5. Full Mediation Model $\left(\mathrm{M}_{1}\right)$ for Production Deviance.................................. 179

Figure 6. Full Mediation Model $\left(\mathrm{M}_{1}\right)$ for Behavior Engagement ............................... 180 


\section{Chapter 1: Introduction}

Organizational commitment has attracted much attention as an attitudinal research topic over the last 20 years (Cohen 2003; Erdheim, Wang, \& Zickar, 2006; Harrison, Newman, \& Roth, 2006; Morrow, 1993). It has been shown that employees with high organizational commitment not only tend to remain longer with their organization but also exhibit more positive on-the-job behaviors (e.g., attendance, task performance and contextual performance), experience more job satisfaction, job involvement, and cope better with stressors at work (Begley \& Czajka, 1993; Harrison et al., 2006; Meyer, Stanley, Herscovitch, \& Topolnytsksy, 2002). There are currently at least three active approaches to measuring organizational commitment: the Organizational Commitment Questionnaire by Mowday, Steers and Porter (1979), the Identification/Internalization Typology by O'Reilly and Chatman (1986), and the Three-Component Model of organizational commitment (TCM) by Meyer and Allen $(1991,1997)$. Among these approaches, the TCM is widely regarded as the most dominant model in organizational commitment research (Bentein, Vandenberg, Vandenberghe \& Stinglhamber, 2005; Cohen, 2003, 2007; Greenberg \& Baron, 2003; McDonald \& Makin 2000; Solinger, van Olffen, \& Roe, 2008).

According to Meyer and Allen (1991, 1997), organizational commitment is a multidimensional construct with three distinct attitudinal components: affective commitment, continuance commitment, and normative commitment. These components represent three psychological states of employees with regard to an organization that influence their decision to maintain membership with the organization. Affective 
commitment is defined as employees' emotional attachment to, identification with, and involvement in the organization. Continuance commitment is defined as employees' awareness of perceived cost of leaving the organization. Finally, normative commitment refers to employees' feeling of obligation to remain with the organization. In other words, employees may remain with the organization because they want to (affective commitment), need to (continuance commitment), or ought to (normative commitment) do so. According to Allen and Meyer (1990), a person's total commitment would reflect the 'net sum' of these three psychological states.

Nonetheless, recent research by Solinger et al. (2008) questioned the legitimacy of the TCM as a general model of organizational commitment. Based on the Theory of Reasoned Action (TRA; Ajzen \& Fishbein, 1980; Fishbein \& Ajzen, 1975), these authors argued that only affective commitment embodies organizational commitment as it represents a general attitude toward an organization. In contrast, continuance and normative commitment correspond to attitudes toward a specific behavior (i.e., the act of leaving) and thus they do not represent organizational commitment. Therefore, grouping qualitatively different components under one general label of attitudinal construct in the TCM would be logically incorrect (Solinger et al., 2008). They suggested that it is important to consider organizational commitment strictly as an attitude toward the organization (i.e., affective commitment). For continuance and normative commitment, it is more appropriate to consider them as antecedents of attitudes toward the act of leaving (i.e., turnover) because they are involved with anticipated consequences of discontinuing employment. This reconceptualization of organizational commitment may help to 
reconcile the inconsistencies found in the TCM's empirical research findings such as the lack of discriminant validity between affective commitment and normative commitment and the low convergent validity of continuance commitment. It is important to note that although Solinger et al. (2008) made a convincing theoretical case to reconceptualize the TCM based on the TRA, no studies so far have empirically tested this conceptualization of organizational commitment.

The purpose of this study was to directly examine the conceptualization of organizational commitment as a singular construct in predicting several important organizational behaviors (i.e., considerate voice, production deviance, and behavioral engagement). I expect to contribute to the literature in three ways. First, the present study will be able to illustrate the nature of organizational commitment by validating the nomological network of its relevant constructs. Second, by having a clearer conceptualization, we will have a better understanding about how organizational commitment is developed and influences organizational behaviors. Finally, by applying this new conceptualization, this study will provide explanations regarding the differences in the relations between different components of the TCM and work-related outcomes documented in the TCM research (Meyer et al., 2002).

In the following chapters, I first provide a summary of the conceptual development of the TCM as well as the empirical findings regarding its antecedents, correlates, and outcomes. Current inconsistencies in empirical research of the model will be highlighted. Second, I review the Theory of Reasoned Action, a well-validated theory of attitude, together with its most recent theoretical development. More specifically, I 
review the model developed by Eagly and Chaiken (1993) which clearly delineates how attitude toward target and attitudes toward behavior influence overt behavior. Third, I describe in detail the organizational behaviors of interest in the current study, including their definitions, related theoretical backgrounds, and the reasons for choosing them. Fourth, I develop specific hypotheses for the current study. Fifth, I describe the methodology of the current study. Sixth, I present the results of my scale validation procedures for new measures in the current study and review the test of my study's hypotheses. Finally, I discuss the theoretical and practical implications of the current study, the limitations, and provide recommendations for future research. 
Chapter 2: The three-component model of organizational commitment In the current chapter, I review the theoretical frameworks from which the TCM originated. More specifically, I review the side-bet approach (Becker, 1960) and the attitudinal approach (Porter, Steers, Mowday, \& Boulian, 1974) to organizational commitment and discuss how these approaches influence the development of the three components under the TCM. Next, I provide a brief review of the empirical findings of the TCM including its antecedents, correlates and outcomes. Finally, I highlight empirical inconsistencies under the TCM, which motivate the current study.

\section{The Development of the TCM}

The development of the TCM is rooted in two earlier approaches to organizational commitment that are often referred as side-bet approach (Becker, 1960) and attitudinal approach (Porter et al., 1974). Becker's (1960) side-bet theory is considered as one of the earliest attempts to conceptualize commitment to explain why people engage in what he called a "consistent line of activity" (p. 33). According to this theory, people participating in social organizations either consciously or unconsciously make various kinds of "sidebets" or investments, which are accumulated over time to restrain their future behaviors. The theory posits that the reason we maintain our membership with an organization is because we fear that, in leaving the organization, we will lose our "side bets". The "sidebets" can be financial loss, loss of promotion opportunity, loss of established relationships and "connections" in the current organization, or loss of ease in doing the job (Becker, 1960). The theory also posits that the more investments one has with an organization, the harder for one to leave the organization (Becker, 1960). 
Becker's side-bet approach implies that organizational commitment can be defined as whether or not an employee maintains his or her membership with an organization (Cohen, 2007). In fact, it argues that organizational commitment is a primary explanatory variable for voluntary turnover. Later research following this approach has attempted to measure organizational commitment by either evaluating the reasons (e.g., increase in pay, status, job freedom, and coworker friendliness) that cause a person to leave the organization (e.g., Alutto, Hrebiniak, \& Alonso, 1973) or using side bets indexes such as age and organizational tenure (e.g., Pfeffer \& Lawler, 1980; Ritzer \& Trice, 1969).

The second approach that also had an important influence on the development of the TCM is that of Porter et al. (1974). Porter et al. switched the focus from commitment as engaging in a consistent line of activity to commitment as an affective or emotional attachment to an organization. Porter and his colleagues argued that Becker's (1960) conceptualization of commitment (i.e., engaging in a consistent line of activity) is actually the "overt manifestations of commitment" rather the commitment itself (p. 225). Accordingly, they defined organizational commitment as "the relative strength of an individual's identification with and involvement in a particular organization" (Mowday et al., 1979, p. 226). Organizational commitment under this approach is characterized by three related factors: “(1) a strong belief in and acceptance of the organization's goals and values; (2) a willingness to exert considerable effort on behalf of the organization; and (3) a strong desire to maintain membership in the organization" (Mowday et al., 1979, p. 226). From this definition, it is obvious that maintaining membership in an organization 
is also an important outcome of organizational commitment conceptualized as an affective or emotional attachment to an organization (Cohen, 2007).

The Organizational Commitment Questionnaire (OCQ) was established to measure organizational commitment as conceptualized by Porter et al. (1974). Although the OCQ was based on the three related factors that characterize organizational commitment, most of researchers considered it as a unidimensional instrument. Despite the fact that the OCQ is the most commonly used organizational commitment scale in the literature (Cooper-Hakim \& Viswesvaran, 2005), it has been criticized for the fact that most of its items tend to reflect a strong desire to maintain membership with an organization which could inflate the relation between organizational commitment and turnover (Bozeman \& Perrewe, 2001; O'Reilly \& Chatman, 1986; Reichers, 1985). As a result, some researchers have adapted the OCQ with select modifications, such as using a 9-item scale without 6 negative worded items (Iverson, 1999), or omitting 3 items supposedly dealing with turnover intentions (Beck \& Wilson, 2000).

The influence of the side-bet and attitudinal approaches to organizational commitment is evident in the development of the TCM. Meyer and Allen (1984) argued that past studies following Becker's (1960) side-bet approach (i.e., Alutto, et al., 1973; Ritzer \& Trice, 1969) have inappropriately operationalized organizational commitment. They argued and empirically showed that the instruments developed by these researchers are instead representative of attitudinal commitment. As a result, they proposed the Continuance Commitment Scale as a better way to measure organizational commitment following Becker's (1960) approach as it directly measures the perceptions of the size 
and the significance of the investments that employees made in their current organizations. Meyer and Allen (1984) proposed the Affective Commitment Scale, which follows the attitudinal approach to organizational commitment, to capture the type of commitment that is distinct from the instrumental worth of maintaining membership with an organization (Buchanan, 1974). The two distinct forms of commitments were conceptualized as being independent such that the extent to which one is affectively committed does not influence his or her level of continuance commitment and vice versa (Meyer \& Allen, 1984). Also, as Solinger et al. (2008) rightly pointed out, these two forms of commitment are different in their targets: affective commitment represents an employee's attitude toward an organization while continuance commitment represents an employee's attitude toward the act of leaving an organization.

Allen and Meyer (1990) later proposed a third form of commitment to which they referred as normative commitment. Normative commitment reflects a perceived obligation to remain with an organization (Meyer \& Allen, 1991). In essence, normative commitment is very similar to what Wiener (1982) referred as the "totality of internalized normative pressures to act in a way which meets organizational goals and interests" (p.471). A normatively committed employee, therefore, will maintain membership with an organization because it is a right and moral thing to do. To this extent, similar to continuance commitment, normative commitment is also an attitude toward the act of leaving an organization. Meyer and Allen (1991) suggested that normative commitment might derive from socialization or exchange processes within organization. Socialization processes refer to the normative pressures exerted on a person before or after entering an 
organization (Wiener, 1982). Exchange processes within organizations, on the other hand, create an obligation in which both sides of the dyadic relationship expect fair and balanced exchanges in term of the favors they do for or receive from others (Blau, 1964). The treatments and supports from the organization will create an obligation for employees to return the favors by maintaining their membership with the organization. Integrating different approaches to organizational commitment, the TCM, which consists of affective commitment, continuance commitment and normative commitment, has become a dominant model of organizational commitment (Cohen, 2007). It is important, however, to note several important features of the TCM that distinguish itself from the previous approaches to organizational commitment. First, the TCM recognizes that organizational commitment is a multidimensional construct and that each of the dimensions of organizational commitment has distinct antecedents and outcomes (Meyer\& Allen, 1991, 1997; Meyer et al., 2002). Second, although commitment conceptualized following Becker's (1960) side-bet approach was often categorized as behavioral commitment (e.g., Meyer \& Allen, 1984; Mowday et al., 1982; Scholl. 1981), Meyer and Allen (1991) later argued that continuance commitment should actually be considered as an attitudinal commitment. The underlying reason for this conceptualization is because Becker's commitment requires a person to recognize or perceive the costs associated with discontinuing a certain type of activity. The conscious recognition or perception of the costs associated with the behavior (i.e., leaving the organization) will have direct influence on the attitude toward that behavior, which will eventually influence the overt behavior (i.e., staying vs. leaving the organization; Meyer 
\& Allen, 1991). Finally, and also most importantly, Meyer and Allen (1991, 1997, see also Allen \& Meyer, 1990) conceptualized the model's dimensions as distinguishable components, rather than types, of organizational commitment and that employees can experience these psychological states to varying degrees. This conceptualization implies that different components of the TCM may interact to influence work behaviors (Meyer, Paunonen, Gellatly, Goffin, \& Jackson, 1989; Randall, Fedor, \& Longenecker, 1990). As will be discussed in more details in the following sections, the combination of components with different qualities (i.e., attitude toward an organization and attitude toward an activity) in a general model of organizational commitment has attracted considerable criticism at both empirical and conceptual levels (Solinger et al., 2008). Empirical Review of the TCM

Voluminous efforts have been devoted to evaluation of the TCM, to test its scales as well as to use them as the main instruments in studying organizational commitment. The objective of this section is to provide a brief review of the antecedents, correlates and outcomes of the TCM. Readers are referred to Meyer et al. (2002) and Cooper-Hakim and Viswesvaran (2005) for a more complete review of the TCM.

Antecedents of the TCM can be generally divided into four main groups: demographic variables, individual differences, work experiences and alternatives/investments (Meyer \& Allen, 1991, Meyer et al., 2002). While demographic variables and individual differences are expected to be related to all commitment components, Meyer and Allen argued that work experiences are the primary antecedents 
of affective commitment and alternative/investments are the primary antecedents of continuance commitment.

Main demographic variables, such as age and tenure, have been shown to be related to affective commitment since the very first study of the TCM (Meyer \& Allen, 1984). The meta-analysis of Meyer et al. (2002) showed that age and tenure are positively, but weakly, related to all three components of commitment with correlations ranging from .12 to .15 for age and from .16 to .21 for organizational tenure. A more recent meta-analysis of $\mathrm{Ng} \&$ Feldman (2010) also found that the relations between age and the three components of commitment were ranging from .20 to .24 . In addition, these meta-analyses also found some interesting moderating effects among demographic variables. More specifically, Ng and Feldman (2010) found that age correlated more strongly with affective commitment (.28 vs. .23) and normative commitment (.34 vs. .07) in samples with higher organizational tenure. They also showed that age was more strongly related to affective commitment in samples with more racial minorities rather than Caucasians (.25 vs. .19). With regard to level of education, $\mathrm{Ng}$ and Feldman found that age was more strongly related to affective commitment (.27 vs. 16) and continuance commitment (.30 vs. .10[ns]) for employees without a college education than for employees with a college education. Finally, Meyer et al. (2002) found that age correlated more strongly with continuance commitment (.20 vs. .12) but less strongly with normative commitment (.07 vs. .15) for studies conducted outside North America; organizational tenure also correlated less strongly with normative commitment for studies conducted outside North America. On a related note, the results also suggest that national 
culture is likely an important contextual factor that may affect the development of organizational commitment and its relationship with organizational and individual outcomes.

In conclusion, the majority of the demographic variables play a relatively minor role in the development of organizational commitment (Mathieu \& Zajac, 1990, Meyer et al., 2002). Among them, however, age is becoming an important variable as the average age of the workforce in the developed world is on the rise. A better understanding of relation between age and organization commitment will help to further our understanding of age - work behaviors relations (e.g., differences in the productivity of older and younger employees) and how organizational commitment changes over time (e.g., whether changes in job attitudes are at least attributable to chronological aging) ( $\mathrm{Ng} \&$ Feldman, 2010).

Individual differences are important in understanding job attitudes such as job satisfaction and organizational commitment (Erdheim et al., 2006). Although there is an increasing body of knowledge supporting the argument that job satisfaction is, at least partially, explained by individual dispositions (House, Shane, \& Herold, 1996), much less is known with regard to the relations between individual differences (e.g., personality) and organizational commitment (Erdheim et al., 2006; Meyer et al., 2002). Among the studies that explored the relations between personality and organizational commitment, a majority of them (e.g., Cropanzano, James, \& Konovsky, 1993; Thoresen, Kaplan, Barsky, Warren, \& de Chermont, 2003) have employed positive affectivity (PA) negative affectivity (NA) typology (Watson, Clark, \& Tellegen, 1988) in their 
investigation. In their recent meta-analysis, Thoresen et al. (2003) found that organizational commitment is positively related to PA (.35) and is negatively related to NA (-.27). Similar correlations (.22 and -.21 respectively) were found when PA and NA are conceptualized as Extraversion and Neuroticism in the Big Five personality framework. In another meta-analysis, Meyer et al. (2002) found that external locus of control had a negative correlation with affective commitment (-.29) while self-efficacy has a weak positive correlation (.11). Finally, Erdheim et al. (2006) provided a more indepth and systematic investigation of the relations between Big Five personality traits (i.e., Extraversion, Neuroticism, Agreeableness, Conscientiousness and Openness to Experience) and organizational commitment. They found that Extraversion was significantly related to all the three components of commitment while Agreeableness was significantly related to only normative commitment. In addition, Neuroticism, Conscientiousness, and Openness to Experience were all significantly related to continuance commitment.

Although we recognize that demographic variables have some impacts on organizational commitment and individual differences predispose employees to a certain level of organizational commitment, work experience variables are considered as the most important group of antecedents of organizational commitment (Meyer et al., 2002). What distinguishes these variables from the previous antecedents of organizational commitment is that they are under the volitional control of organizations and their agents (e.g., supervisors and managers). Accordingly, measures can be taken by organizations to influence their employees' organizational commitment. Important work experience 
variables include, yet are not limited to, concepts (a) intrinsic to the job, such as skill variety, autonomy, job scope; (b) related to individual's organizational roles, such as role ambiguity and role conflict; (c) of an organizational nature, such as organizational support, culture, and HR strategies; (d) concerned with individual's work relationship, such as social support, leadership, and organizational justice; and (e) concerned with individual career development such as job security and advancement potential (Mathieu \& Zajac, 1990; Quick, 1999). Within research involving the TCM, it has been found that work experience variables have the strongest relations with affective commitment, and to a lesser extent, with normative commitment, and the weakest relations with continuance commitment (Meyer et al., 2002). Also, in addition to having weak correlations with work experiences, all the correlations involving continuance commitment are of opposite signs of those involving affective and normative commitment (Meyer et al., 2002). For this reason, I will mainly focus on the relations between work experience variables and affective and normative commitment.

Organizational support has been argued to be the most important antecedent of affective commitment. In a recent meta-analytic review of the TCM, organizational support was found to be the strongest predictor of affective commitment (.63) and to a lesser extent of normative commitment (.47) (Meyer et al., 2002). One of the ways that organizations can give support to employees is by providing strong leadership. In fact, transformational leadership was found to be related strongly to affective commitment (.46) and moderately to normative commitment (.27). Another way to provide support to employees is to treat them fairly. Employees' perception of fairness is also a major 
predictor of the TCM. Various forms of organizational justice were found related to affective commitment ( .40 for distributive justice, .38 for procedural justice and .50 for interactional justice) and normative commitment ( .31 for distributive justice, .31 for procedural justice and .52 for interactional justice) (Meyer et al., 2002). On a related topic, research also has found that the violation of the psychological contract on the part of employers will lead to decrement of affective commitment (e.g., Bunderson, 2001; Cassar, 2001; Cassar \& Briner, 2010; Coyle-Shapiro \& Kessler, 2000; Guzzo, Noonan, \& Elron, 1994); and vice versa, the fulfillment of employer's obligations will enhance employee affective and normative commitment (e.g., McInnis, Meyer \& Feldman, 2009). In an opposite direction, various individual's role states (e.g., role ambiguity and role conflict) were found to be negatively related both to affective commitment (-.39 for role ambiguity and -.30 for role conflict) and normative commitment (-.21 for role ambiguity and -.24 for role conflict).

Finally, researchers also have tested the relations between human resource (HR) practices and organizational commitment. The findings, however, are inconclusive. While there are some evidences suggesting that HR practices do influence organizational commitment (e.g., Gong, Law, Chang, \& Xin, 2009; Hung, Ansari, \& Aafaqi, 2004), a recent study by Fiorito, Bozeman, Young, and Meurs (2007), using a diverse national sample of U.S. workers and organizations, provided limited support for the proposed effects of HR practices. Although Fiorito et al. (2007) used the Organizational Commitment Questionnaire by Mowday et al. (1979) for measuring organizational commitment, OCQ is conceptually and empirically similar to affective commitment ( $r=$ 
.88) (as shown in Allen \& Meyer, 1997; Meyer et al., 2002). There are several reasons for the unsettled conclusion in this area of research. First, the HR practices measures are often unique and not comparable across studies which make comparison and accumulation of findings difficult at best (Fiorito et al., 2007). For example, Whitener (2001) followed Snell and Dean's (1992) approach to measuring HR practices in term of how "committed" they are. In addition, Allen, Shore, and Griffeth (2003) proposed a conceptualization of HR practices in term of how "supportive" they are. Yet, others prefer to operationalize HR practices in term of its functions, such as hiring, training, or compensation (e.g., Fiorito et al. 2007), or to classify HR practices as under promotionoriented vs. maintenance-oriented focus (e.g., Gong et al., 2009). Second, the mixed findings in the literature may result from the presence of moderators. For example, there is evidence that suggests the quality of a relationship between leader and member (i.e., Leader-Member Exchange) moderates the relations between HR practices and affective commitment (Hung et al., 2004). In addition, recent study by Kwon, Bae, and Lawler (2010) found that the effects of HR practices on affective commitment are stronger for high performing employees than for low performing employees. Finally, the nonsignificant effects can be attributable to that fact that HR practices variables are often conceptualized at the organizational level and are far removed from individual attitudinal variables. Research suggests that HR practices and organizational characteristics may affect organizational commitment only through the more proximal processes such as group interaction, perceived organizational support or fairness perception (Eisenberger, 
Huntington, Hutchison, \& Sowa, 1986; Mathieu \& Zajac, 1990; Meyer \& Smith, 2000; Pare \& Tremblay, 2007).

Research in the development of affective commitment from work experiences has departed from what Reichers (1985, p. 467) referred to as a 'laundry list' of significant antecedents. Multiple mechanisms through which work experience variables impact on affective commitment have been proposed such as Organizational Support Theory (Eisenberger et al., 1986), Psychological Contract (Rousseau, 1989), and Prosocial Sensemaking Process (Grant, Dutton, \& Rosso, 2008). Among those approaches, Organizational Support Theory has received most research attention and support. The theory is based on the norm of reciprocity which regulates the exchange relationship between employers and employees and reconciles their differing objectives (e.g., maximizing performance vs. maximizing rewards) to achieve better outcomes for both parties (Rhoades \& Eisenberger, 2002). When the reciprocity norm is at work, it obligates both parties to maintain a fair and balanced exchange in their dyadic relationship (Gouldner, 1960). In other words, when employees received favorable treatment and support from employers in term of tangible (e.g., pay, bonuses, or promotion) and intangible (e.g., recognition or treatment with fairness and respect) benefits, employees are obligated to reciprocate by providing the organization with commitment, loyalty, good performance, and other on-the-job behaviors that benefit the organization (Rhoades \& Eisenberger, 2002).

In order to determine the organization's readiness to meet the financial and socioemotional needs, employees develop Perceived Organization Support (POS) which 
is a global belief concerning the extent to which organizations commit to and care about them (Eisenberger et al., 1986). The assessment of the quality of support from the organization will then influence the attitudes, and eventually, the behaviors of employees at work (Wayne, Shore, Bommer, \& Tetrick, 2002; Wayne, Shore \& Liden, 1997). Eisenberger and his colleagues posit the mechanism through which POS is heightened and influences job attitudes and work behaviors consists of three processes. First, under the reciprocity norm, favorable treatments from organizations and their agents will create a felt obligation to reciprocate by caring more about organization's survival and wellbeing. Second, these favorable treatments also fulfill social and emotional needs of employees. This is particularly important because work has such a profound impact on our lives more than any other kinds of activities that we may do or social roles that we may take (Hulin, 2002). Third, POS should increase the performance-reward expectancies because of the recognition and rewards provided by the organizations.

Rhoades and Eisenberger (2002), in their meta-analysis of more than 70 empirical studies of POS, found that POS is strongly related to fairness of treatment (.68), supervisor support (.64), and organizational rewards and job conditions (.46). They also found that affective commitment is strongly related to POS (.73). Several studies (e.g., Hofmann \& Morgeson, 1999; Masterson, Lewis, Goldman, \& Taylor, 2000; Setton, Bennett, \& Liden, 1996; Wayne et al., 1997, 2002) using Structural Equation Modeling have found evidence supporting the mediating role of the POS between various work experience variables (e.g., fairness perception, leader-member exchange, developmental experiences, inclusion and recognition) and affective commitment. 
The last group of antecedents of the TCM is alternatives/investments. These variables are expected to be related more strongly with continuance commitment than with affective or normative commitment because continuance commitment in the TCM is conceptualized following Becker's (1960) side-bet approach (Meyer \& Allen, 1991). Meta-analytic results indicated that the availability of alternatives to current jobs had a relatively stronger relation with continuance commitment (-.21) compared with affective and normative commitment (-.07 and -.08 respectively; Meyer et al., 2002). On a related note, Meyer et al. also found that continuance commitment increased if employees did not believe that their education and skills could be easily transferred to alternative organizations. More specifically, perceived transferability of skills and education were more strongly related to continuance commitment (-.31 and -.22 respectively) compared with affective commitment (.17 and -.04) and normative commitment (.13 and -.07). Finally, however, the relations between investments and these commitment components did not follow the same pattern. Investments correlated more strongly with affective (.24) and normative (.21) commitment in comparison with continuance commitment (.01).

While there are still various unresolved issues with the development of organization commitment in the TCM (e.g., the development of continuance and normative commitment), the research on its outcomes has been well established. High organizational commitment has been associated with positive outcomes valued by both employers and employees such as lower turnover intention and turnover, positive on-thejob behaviors (e.g., helping others, working extra hours, information sharing, quality concerns and supervisor evaluation of performance), and better health and well-being 
(Meyer \& Herscovitch, 2001; Solinger et al, 2008). Turnover and turnover intention have been the primary outcomes under the TCM. In fact, a common notion among all three model's components is the "psychological state that links an individual to an organization (i.e., makes turnover less likely)" (Allen \& Meyer, 1990, p. 14). Meyer et al. (2002) found that turnover intention was correlated most strongly with affective commitment ($.56)$, followed by normative (-.33) and continuance (-.18) commitment. In a similar pattern but much lower in strength, they found that actual turnover correlated most strongly with affective commitment (-.17), followed by normative (-.16) and continuance (-.10) commitment. In a more recent meta-analysis, Cooper-Hakim and Viswesvaran (2005) found a similar result for the relations between turnover intention and the three commitment components (-.58 for affective commitment, -.37 for normative commitment, and -.19 for continuance commitment). However, a different pattern of correlations was found for actual turnover showing that it correlated most strongly with continuance commitment (-.25), followed by affective (-.20) and normative (-.16) commitment.

In term of job performance, Meyer et al. (2002) found that affective and normative commitment correlated positively with job performance (.16 and .06 respectively) but continuance commitment correlated negatively with job performance (.07). Cooper-Hakim and Viswesvaran (2005) found a similar result for the relations between job performance and continuance (-.09) and normative (.06) commitment, albeit slightly larger for affective commitment (.22). With regard to contextual performance, Meyer et al. found that Organizational Citizenship Behaviors (OCB) correlated positively 
with affective (.32) and normative (.24) commitment while its relation with continuance commitment was near zero. Two conclusions can be drawn from these results. First, while continuance commitment may help to keep the employees with the organization, the feeling of entrapment (i.e., have to stay with the organization) will eventually hurt their task performance. Second, affective and normative commitment correlated more strongly with OCB than with task performance. This result might be explained by the fact that task performance is considered as part of the job requirements and OCB is more "discretionary, not directly or explicitly recognized by the formal reward system" (Organ, 1988, p. 4). Employees with high affective and normative commitment will go beyond what is required on the job and do things that contribute to "the maintenance and enhancement of the social and psychological context that supports task performance" (Organ, 1997, p. 91). Finally, organizational commitment has also been linked to outcomes that matter to individuals rather than organizations (e.g., stress and work-family conflict). Meyer et al. (2002) found that affective commitment correlated negatively with stress (-.21) and work-family conflict (-.20) while continuance commitment correlated positively with these outcomes (.14 and .24 respectively).

Besides antecedents and outcomes, research in organizational commitment recognizes another group of variables as correlates of affective commitment (e.g., Mathieu \& Zajac, 1990; Meyer et al., 2002). The reason to consider certain variables such as job involvement and job satisfaction as correlates is because no consensus regarding the causality between them and affective commitment exits. Meyer et al. (2002) found that affective commitment correlated strongly with overall job satisfaction (.65), although 
the correlations with more specific facets of satisfaction were lower. In addition, they also found that affective commitment correlated positively with job involvement (.53) and occupational commitment (.51). Cooper-Hakim and Viswesvaran (2005) found a similar result: affective commitment correlated strongly with job satisfaction (.60), job involvement (.50), and occupational commitment (.61).

Empirical and Conceptual Issues with the TCM

Despite the popularity and initial support of the TCM, there has been an accumulation of empirical evidence showing that the model does not consistently hold up to rigorous testing or analytical scrutiny (Allen \& Meyer, 1996; Ko, Price, \& Mueller, 1997; McGee \& Ford, 1987; Meyer et al., 2002). Two issues identified through empirical research of the TCM, as discussed by Allen and Meyer (1996; see also Meyer et al., 2002), are (a) the low convergent validity of continuance commitment and (b) the lack of discriminant validity between affective commitment and normative commitment.

Continuance commitment is often criticized for its lack of convergent validity with affective commitment, important correlates and behavioral outcomes (Cohen, 2003; Cooper-Hakim \& Viswesvaran 2005; Meyer et al., 2002; Solinger et al., 2008). Meyer et al. (2002) found continuance commitment correlates only weakly with affective commitment (.05) and slightly negatively or not at all with job satisfaction (-.07), job involvement (.03), job performance (-.07) and OCB (-.01). McGee and Ford (1987) attributed the weak correlation between continuance commitment and affective commitment to the fact that the relations between affective commitment and two subdimensions of continuance commitment (i.e., lack of alternatives and high sacrifices) are 
similar in magnitude but of opposite directions. Finally, in their meta-analysis of work commitment, Cooper-Hakim \& Viswesvaran (2005) concluded that continuance commitment can be regarded as a distinct construct from the general work commitment construct because it reflects "a looser coupling of an individual with the workplace" (p. 253).

The second empirical issue relating to the TCM is the relation between normative and affective commitment. Meyer et al.'s (2002) meta-analysis found a correlation of .63 between affective and normative commitment. A similar correlation of .64 between these constructs was reported in a more recent meta-analysis by Cooper-Hakim and Viswesvaran (2005). In addition, as indicated in the empirical review of the TCM, normative and affective commitment also share a large number of antecedents, correlates and outcomes (Meyer et al., 2002). Although Meyer and Allen (1991) argued that the two constructs are more different in "tone" and rather behavioral outcomes, it could be argued that "tone" would be too ambiguous and conceptually elusive to be qualified as a basis for differentiation between two constructs. In conclusion, meta-analytic evidences suggest that there is a considerable overlap between two constructs and it is hard to separate them empirically (Ko et al., 1997). In other words, affective and normative commitment are likely to suffer from concept redundancy, a major problem in the work commitment literature (Cooper-Hakim \& Viswesvaran, 2005; Morrow, 1983, 1993).

Attentive to these issues, attempts have been made by Meyer and his associates to alter the operationalization of the TCM's continuance and normative commitment. For example, Meyer, Allen, and Smith (1993) proposed a modification of the normative 
commitment scales developed by Allen and Meyer (1990) by focusing more on the employees' sense of obligation to maintain membership with an organization. Lee, Allen, Meyer and Rhee (2001) proposed another modification of continuance and normative commitment scales developed by Meyer et al. (1993) in order to be able to generalize the TCM to another culture (i.e., South Korea). With regard to the two sub-dimension controversy of continuance commitment, Powell and Meyer (2004) proposed another new continuance commitment scale that contains only items measuring the high sacrifices sub-dimension. More recently, Meyer and Parfyonova (2009) attempted to reconceptualize normative commitment by arguing that that normative commitment has a "dual nature and that it manifests itself differently depending on the strength of other components in an employee's commitment profile" (p. 283). More specifically, they argued that when normative commitment is coupled with high affective commitment it reflects a sense of moral duty - a desire to engage in a certain line of activities that benefit the organization because it is a right and moral thing to do. On the other hand, when normative commitment is coupled with high continuance commitment, it reflects a sense of indebted obligation - an obligation to engage in a certain lines of activities that benefit the organization to avoid the anticipated costs of failing to do so. The different nature of normative commitment is proposed to have different implications for work behavior (Meyer \& Parfyonova, 2009). Finally, TCM researchers also attempted to conceptually modify the attitudinal nature of the components to motivational one (e.g., Meyer, Becker, \& Vandenberghe, 2004; Meyer \& Herscovitch, 2001). While these modifications did improve some of the psychometric properties of the scales, there is no 
consensus on which scales should be used in research (Cohen, 2007). In addition, certain propositions (e.g., Meyer \& Parfyonova, 2009) have yet to be tested empirically.

Although most criticism of the TCM have been involved with empirical issues, several researchers (e.g., Ko et al., 1997; Solinger et al., 2008) have suggested that the TCM suffers a more serious conceptual shortcoming. These authors argued that affective, continuance, and normative commitment are attitudes with different qualities. More specifically, affective commitment is an attitude defined by its target (i.e., an organization) while continuance and normative commitment are attitudes defined by their actions (i.e., the act of leaving an organization). Therefore, grouping these components under one general label of attitudinal construct in the TCM would be logically incorrect (Solinger et al., 2008).

\section{Chapter Summary}

The current chapter reviewed the theoretical backgrounds from which the TCM originated. As discussed, the three components of the TCM originated from very different theoretical frameworks with different assumptions and mechanisms. It suggested that the combination of these components under the same attitudinal label could be problematic. The review of the TCM's empirical findings highlighted the importance of work experience and the process by which affective and normative commitment are developed. TCM's empirical findings also suggest that continuance commitment is only loosely coupled with the other two components. Finally, the review provided a critical review of the empirical inconsistencies under the TCM as well as recent attempts by Meyer and his colleague to overcome the deficiencies of the model. 
Chapter 3: Theory of reasoned action and conceptualization of organizational commitment

In the current chapter I first review the theoretical background and the models of Theory of Reason Action (TRA; Ajzen \& Fishbein, 1980; Fishbein \& Ajzen, 1975) and its recent development, Theory of Planned Behavior (TPB; Ajzen 1985, 1991). Next, I provide a detailed description of Eagly and Chaiken's (1993) model from which the hypothesized model of the current study originated. Eagly and Chaiken's (1993) model is another extension of the TRA which takes into account both attitude toward behavior and attitude toward target in predicting various behavioral outcomes. Finally, I explain how we can use the Eagly and Chaiken's model to diagnose the TCM and reconceptualize organizational commitment.

Attitude and its relation with behavior have been well studied and are of a longstanding interest in the history of social psychology (Eagly \& Chaiken, 1993; Manstead, 1996). In fact, attitude has long been regarded as the most distinctive and indispensable concept in social psychology (Allport, 1935). Attitude is defined as "a psychological tendency that is expressed by evaluating a particular entity with some degree of favor or disfavor" (Eagly \& Chaiken, 1993, p. 1). In other words, attitude is a latent and relatively enduring construct that is internal to a person regarding a distinguishable object (e.g., an organization, social groups, social policies or ideologies) which can be inferred through observable affective, cognitive and behavioral responses (Eagly \& Chaiken, 1993; Manstead, 1996).

The TRA is regarded as one of the most dominant and well-validated theories on attitudinal research (Solinger et al., 2008). The original version of the TRA (Ajzen \& 
Fishbein, 1980; Fishbein \& Ajzen, 1975) argued that behavior is directly determined by one's intention to engage in the behavior. Subsequently, intention is a function of (a) attitude toward the behavior (i.e., one's evaluation of the behavior in question) and (b) subjective norm, (i.e., one's belief about whether significant others think one should engage in the behavior). Furthermore, attitude toward the behavior is jointly determined by behavioral beliefs (i.e., anticipated consequences of the behavior), and outcome evaluation (i.e., evaluation of the consequences of behavior). Subjective norm is also jointly determined by normative beliefs (i.e., beliefs that significant others expect one to behave in a certain way) and motivation to comply (i.e., one's inclination to conform to others' expectation). It is important to note that the original version of the TRA does not include attitudes toward targets in its model. According to Fishbein and Ajzen (1975), this class of attitude and other variables affect behaviors only through the more proximal determinants specified by the original model (Eagly \& Chaiken, 1993; Manstead, 1996). Ajzen $(1985,1991)$ provided an extension of the TRA by adding perceived behavior control to the original model of the TRA and called the new model the Theory of Planned Behaviors (TPB). Perceived behavioral control is defined as "people's perception of the ease or difficulty of performing the behavior of interest" (Ajzen, 1991, p. 183). The development of the TPB was based on an observation that most of our behaviors are not under complete volitional control. The perception of behavioral control, therefore, should add incremental validity in predicting behavioral intention and actual behaviors. Perceived behavioral of control is further a function of control beliefs. According to the TBP, perceived behavioral control is different from one's actual control 
(i.e., the availability of requisite opportunities and resources to perform the behavior) and Rotter's (1966) perceived locus of control (i.e., the generalized expectancy in control that is stable across situations and types of behaviors). Ajzen (1991) argued that perceived behavioral control is similar to the expectancy component (i.e., perceived probability of succeeding at a given task) under Atkinson's (1964) theory of achievement motivation or even more compatible with Bandura's (1977) concept of perceived self-efficacy which defined as "judgments of how well one can execute courses of action required to deal with prospective situations" (Bandura, 1982, p. 122)

Since its formulation, the TRA's model and its variant (e.g. TPB) have attracted considerable research attention and is generally supported in predicting various behaviors such as abortion (Smetana \& Adler, 1980), blood donation (Charng, Piliavin, \& Callero, 1988; Pomazal \& Jaccard, 1976), church attendance (King, 1975), dental hygiene behavior (McCaul, O’Neill, \& Glasgow, 1988; Toneatto \& Binik, 1987), doing exercise (Terry \& O’Leary, 1995), family planning (Crawford \& Boyer, 1985; Davidson \& Jaccard, 1975), drug use (Ajzen, Timko, \& White, 1982; McMillan and Conner, 2003), and voting (Ajzen \& Fishbein, 1980; Fishbein, Middlestadt, \& Chung, 1986). The advantage of the TRA model lies in its ability not only to predict behaviors but also to explain the mechanism of how people engage in certain behaviors (Manstead, 1996).

Eagly and Chaiken (1993) extensively reviewed the key components, developments and other variants of TRA, and extended this theory into a model that takes into account both attitude toward behavior and attitude toward target in predicting various behavioral outcomes. In this model, attitude toward behavior is the central component 
that directly leads to intention and subsequent behavior. The attitude toward behavior is theorized to originate from habit, attitude toward target, and three different classes of anticipated outcomes of the behavior: utilitarian, normative and self-identity. Habits should be understood as sequences of behaviors that occur automatically without effort (Triandis, 1977, 1980). Attitude toward target consists of a general evaluation of the target of the behavior without specific action, context and time. Examples of attitude toward targets are attitude toward a political party, attitude toward labor union, or attitude toward an organization. Anticipated outcomes are the evaluations of potential consequences of behavior. More specifically, utilitarian outcomes refer to the anticipated rewards and punishments for individuals as a result of engaging in the behavior (Eagly \& Chaiken, 1993). In Fishbein and Ajzen's (1975) model, utilitarian outcomes are behavioral beliefs. Normative outcomes concern the anticipated approval or disapproval of significant others in relation to engaging in the behavior (Ajzen \& Fishbein, 1980). Normative outcomes are similar to normative beliefs in Fishbein and Ajzen's (1975) term. Finally, self-identity outcomes are the anticipated feelings of affirmation or repudiation of one's self-concept in engaging in the behavior (Charng et al., 1988). More specifically, self-identity outcomes in the current model refer to whether one is identified with the behavior not. It is important to note that, according to this model, attitude toward target can affect a wide range of organizational behaviors toward the target while anticipated outcomes of one specific behavior (e.g., the act of leaving) will not affect the probability of engaging in other behaviors (e.g., contextual performance). Also, the model argues that attitude toward target will not directly affect behaviors, but will do so 
via a cognitive process which translates the general attitude toward the target to attitudes toward specific behaviors (Eagly \& Chaiken, 1993; Fazio, 1986). In order to avoid the confusion between the three classes of anticipated outcomes (i.e., evaluations of potential consequences of behavior) and behavioral outcomes (i.e., organizational behaviors), from this point forward, I will use behavioral expectancies (e.g., utilitarian expectancies, normative expectancies, and self-identity expectancies) to talk about the three classes of anticipated outcomes of behaviors.

The new conceptualization of organizational commitment proposed by Solinger et al. (2008) is clearly illustrated by applying the Eagly and Chaiken's model to reconceptualize the TCM. First of all, because affective commitment is defined as “employee's emotional attachment to, identification with and involvement in the organization (Meyer \& Allen, 1991, p. 67), it clearly represents an attitude toward a target (i.e., organization ${ }^{1}$ ) in Eagly and Chaiken's model. Second, continuance commitment refers to the "awareness of the costs associated with leaving the organization” (Meyer \& Allen, 1991, p. 67). It apparently corresponds with utilitarian expectancies which refer to the anticipated punishments as outcomes associated with the act of leaving the organization. Finally, normative commitment, "a feeling of obligation to continue employment" (Meyer \& Allen, 1991, p. 67) because of internalized moral obligation (Wiener, 1982) or expectation of significant others, could be considered as the

\footnotetext{
${ }^{1}$ It is important to note that while employees do have attitude toward their supervisors, they tend to attribute the actions of organizational agents (e.g., supervisors) as organizational intentions, which included in affective commitment (Levinson, 1965). Similarly, employees also have attitude toward their coworkers, however, since I focus on organization-directed behaviors, attitude toward coworker is not included in the current model. Future research predicting behaviors directed to individuals (e.g., bullying or incivility behaviors) may incorporate attitude toward coworkers in the model.
} 
combination of both normative expectancies and self-identity expectancies of leaving the organization (Solinger et al., 2008).

In the current study, I apply Eagly and Chaiken's model for testing antecedents of multiple organizational behaviors with two adjustments. The first adjustment is that I do not include the intention component in the model for the current study. This is because I am only interested in detecting the effects of different attitudinal constructs in predicting organizational behaviors. Not including the intention component as a mediator would not influence my estimations of these effects. Further, if measured at the same time, the relation between attitude and intention has been argued to be prone to common method bias (Podsakoff, MacKenzie, Lee, \& Podsakoff, 2003). To minimize the potential common method bias, I would have to measure intention at a different time, which would make the current two-wave longitudinal design become three-wave, adding more items and extra burden in conducting the study, which might reduce the response rate. The second adjustment is that I choose not to include the habit component in the current investigation because it represents an automatic and subconscious process in leading to behaviors, which is not the focus of the current prediction (Triandis, 1977, 1980). Figure 1 illustrates the modified model for the current study.

\section{Chapter Summary}

The review of Theory of Reasoned Action, especially Eagly and Chaiken's (1993) model, indicated that TRA is an appropriate theoretical framework to reconceptualize organizational commitment stipulated by the TCM. Eagly and Chaiken's model is helpful because it takes into account both attitude toward target and attitude toward behavior in 
predicting behaviors. The reconceptualization of organizational commitment following the TRA has the potential to reconcile the empirical inconsistencies of the TCM and provide a parsimonious model to predict a wide range of behaviors that relevant to both organizations and employees. As mentioned, a modified model of Eagly and Chaiken's model (see Figure 1) were used to generate the hypotheses of the current study. 
Chapter 4: Organizational behavior criteria and hypothesis development

In this chapter, I first provide a detailed description of the organizational behaviors of interest in the current study, including their definitions, the related theoretical backgrounds, and the reasons for choosing them. Second, based on the newly modified model of Eagly and Chaiken's model, hypotheses for the current study will be generated. Figure 1 provides a visual representation of my hypothesized model.

\section{Organizational Behavior Criteria}

For the purpose of the current study, my aim was to choose behavioral criteria that are diverse in their nature and characteristics (thus ensuring the operationalization of attitudes toward multiple types of specific behaviors), but also are important to the organizations. I follow the suggestion from Solinger et al. (2008) to select for organization behavior criteria based on a two-dimensional typology of a constructivedestructive dimension and an active-passive dimension (Hirschman, 1970; Farrell, 1983; Hagedoorn, Van Yperen, Van de Vliert, \& Buunk, 1999). Constructive behaviors are behaviors that typically result in positive outcomes to the organization, whereas destructive behaviors are those that have negative impacts on organizational outcomes. Active behaviors are (often impulsive) acts immediately directed toward their intended target (e.g., yelling at a supervisor; helping a colleague with a task) while passive behaviors are longer-term behavioral patterns that generally affect the target less noticeably (Spector \& Fox, 2002). These are the common dimensions that allow comparisons among different organizational behaviors (Farrell, 1983; Hagedoorn et al., 1999). Showing quality concern (Randall et al., 1990) and prosocial behavior (O'Reilly 
\& Chatman, 1986) are examples of constructive and active behaviors, while loyalty and patience (Farrell, 1983) are examples of constructive and passive behaviors. On the destructive side, workplace aggression (e.g., bullying, verbal and sexual harassment) are examples of destructive and active behaviors, while working to rule and withholding information (Randall et al., 1990) are examples of destructive and passive behaviors. In addition, I selected, in order to extend the literature, criteria organizational behaviors that have not been examined relative to organizational commitment variables. Further, I also took into account the current focus of Industrial/Organizational (I/O) psychology literature on behavioral constructs, for example engagement, in deciding the specific criteria to use in the current study. As a result, my research investigation considers three distinct behaviors to serve as criteria in the current study: considerate voice, production deviance, and behavioral engagement.

Considerate voice is one of the categories of employees' responses to problematic events in an organization (Hagedoorn et al., 1999). Considerate voice consists of behaviors relating to problem solving at work that takes into consideration both the individual's concerns and those of the organization, as opposed to an individuals' effort to win at the cost of the organization. It represents a constructive and active way for employees to influence their environment. It has not been linked to employees’ organizational commitment before.

Production deviance is a specific type of Counterproductive Work Behavior $(\mathrm{CWB})$ in the CWB taxonomy developed by Spector and his colleagues (Penney \& Spector, 2005; Spector \& Fox, 2005; Spector, Fox, Penney, Bruursema, Goh, \& Kessler, 
2006). Production deviance is a "purposeful failure to perform job tasks effectively the way they are supposed to be performed" (Spector et al., 2006, p. 449). Therefore, it is destructive and passive in nature. Production deviance might indirectly cause serious harms to both employees and the organization. A literature search I conducted found no study that looked at specific relation between production deviance and organizational commitment.

While engagement is a very popular construct among practitioners, it is a rather new construct in I/O research (Macey \& Schneider, 2008). According to Macey and Schneider, engagement is a multidimensional construct with three different dimensions: psychological state engagement, behavioral engagement, and trait engagement. Behavioral engagement is theorized to combine some forms of organizational citizenship behavior, job involvement, and proactive/initiative forms of behaviors, therefore, it positive and active in nature. Because behavioral engagement has been generating much attention in $\mathrm{I} / \mathrm{O}$ psychology recently, I include it as one of the criteria in the current study and follow Macey and Schneider's (2008) approach to operationalize it. Hypotheses Development

In the Figure 1, affective commitment (i.e., employees' attitude toward the organization) predicts a wide range of work-related behaviors that impact on organizations (Solinger et al., 2008). And. in fact, recent meta-analyses (e.g., Allen \& Meyer, 1996, Mathieu \& Zajac, 1990, Meyer et al., 2002) have shown that affective commitment correlates to a wider range of behaviors than do continuance or normative commitment. Moreover, since continuance and normative commitment are attitudes 
toward the act of leaving, theoretically, I do not expect them to relate to any of criteria in the current study. For example, "engaging behaviors" should not be predicted by the "awareness of the cost of leaving" or "felt obligation to stay". In general, I expect employees with higher affective commitment are less likely to engage in destructive behaviors and more likely to engage in behaviors that are beneficial to the organization. However, according to Fishbein and Ajzen (1974), it seems likely that affective commitment will predict constructive (positive) behaviors (here, i.e., considerate voice and behavioral engagement) more so than destructive (negative) behaviors (here, i.e., production deviance). Specifically, I predicted that:

H1a: Employees' affective commitment would be positively related to their actual use of considerate voice

H1b: Employees' affective commitment would be negatively related to their actual use of production deviance

H1c: Employees' affective commitment would be positively related to their actual use of behavioral engagement

Solinger et al. (2008) also suggested that not only should we return to the definition of organizational commitment as an affective or emotional attachment to the organization, but organizational behaviors could be better explained by adding several different classes of behavioral expectancies (i.e., utilitarian, normative and self-identity expectancies) of behaviors as behavioral antecedents. However, as discussed earlier, according to the principle of compatibility, the relationship of a given behavioral expectancy to one behavior (e.g., considerate voice) should not necessary relate to its 
relationship to other distinctive behaviors (e.g., production deviance) (Ajzen \& Fishbein, 1977).

Utilitarian expectancies represent the potential rewards and punishments associated with the behaviors. The underlying mechanism of the relation between utilitarian expectancies and behaviors simply follow the expectancy-value paradigm (Eagly \& Chaiken, 1993; Scholl, 1981). Individuals have tendency to engage in behaviors that are more likely to bring rewards rather than punishments. In other words, individuals will weigh the potential gains against the potential costs of engaging in certain behaviors and will engage in behaviors that bring more gains than costs. Therefore, I predict:

H2a: Employees' perception of the utilitarian expectancies of considerate voice would be positively related to their actual use of considerate voice

H2b: Employees' perception of the utilitarian expectancies of production deviance would be positively related to their actual use of production deviance

H2c: Employees' perception of the utilitarian expectancies of behavioral engagement would be positively related to their actual use of behavioral engagement

Normative expectancies refer to the anticipated approval or disapproval from others in relation to engaging in certain behaviors. Research has shown that need for approval is an important motivational force (e.g., Millham, 1974; Rudolph, Caldwell, \& Conley, 2005). Because approval from one's social groups is important, individuals will compare the value of approval against the value of disapproval from people surrounding 
them (Ajzen \& Fishbein, 1980) and are more likely to engage in the behaviors that can bring more approval. Therefore, I expect:

H3a: Employees' perception of the normative expectancies of considerate voice would be positively related to their actual use of considerate voice

H3b: Employees' perception of the normative expectancies of production deviance would be positively related to their actual use of production deviance

H3c: Employees' perception of the normative expectancies of behavioral engagement would be positively related to their actual use of behavioral engagement

Self-identity expectancies are the anticipated feelings of affirmations or repudiations of one's self-concept in engaging the behavior. According to Stryker's identity theory, individuals can be defined in term of their social identities, which are "reflexively applied cognitions in the form of answers to the question 'Who am I?'” (Stryker \& Serpe, 1982, p. 206). For each identity, there is an identity commitment which reflects the quantity and quality the social ties upon which each identity is predicated. Since work plays a very important role in our lives (Hulin, 2002), work-identity thus is among the most salient identities that individuals have (Whitbourne, 1996). Therefore, positive work identity (e.g., a good member of the organization) will motivate individuals to invest more time and effort in the enactment of the identity. In a similar fashion, roleidentity theory by McCall and Simmons (1978) also posits that individuals have the motivation to maintain the identity that is important to them. According to the 
constructive-destructive distinction of behaviors, it is logical to expect that the perception of positive work-identity will encourage constructive behaviors while the perception of negative work-identity will discourage destructive behaviors. Therefore, I predict:

H4a: Employees' perception of the positive self-identity expectancies of considerate voice would be positively related to their actual use of considerate voice

H4b: Employees' perception of the negative self-identity expectancies of production deviance would be negatively related to their actual use of production deviance

H4c: Employees' perception of the positive self-identity expectancies of behavioral engagement would be positively related to their actual use of behavioral engagement

Finally, according to the conceptual model in Figure 1, affective commitment will not directly impact behaviors but instead will influence them through attitude toward specific behaviors. The logic underlying this mechanism is that attitude toward the target will need to transfer into a positive attitude toward the behaviors before having any impact on the behaviors (Eagly \& Chaiken, 1993). This mechanism is also consistent with the principle of compatibility (Ajzen \& Fishbein, 1977) which suggests that the attitudinal construct should be compatible with the behaviors to have any significant relation. Thus, affective commitment, a general attitude toward the organization, will not be highly related to the more specific behaviors in the current study while attitude toward specific behaviors will be highly related to the behaviors themselves. Empirically, this 
means that the relation between affective commitment and actual behaviors should be fully mediated by attitudes toward behaviors. In addition, a similar mediating mechanism also is expected for the three classes of behavioral expectancies. Positive or negative expectancies of engaging in a certain behavior will first make individuals have positive or negative attitudes toward that behavior, which then influences whether they carry out the behavior or not. Therefore, I predict

H5a: Attitudes toward specific behaviors would mediate the relation between affective commitment and the actual use of the three tested behaviors (i.e., considerate voice, production deviance, and behavioral engagement)

H5b: Attitudes toward specific behaviors would mediate the relation between the utilitarian expectancies and the actual use of the three tested behaviors (i.e., considerate voice, production deviance, and behavioral engagement)

H5c: Attitudes toward specific behaviors would mediate the relation between the normative expectancies and the actual use of the three tested behaviors (i.e., considerate voice, production deviance, and behavioral engagement)

H5d: Attitudes toward specific behaviors would mediate the relation between the self-identity expectancies and the actual use of the three tested behaviors (i.e., considerate voice, production deviance, and behavioral engagement)

In summary, I have argued that affective commitment as a sole indicator of organization commitment should predict wide range of behaviors, over and beyond continuance and normative commitment. In addition, I argued that the addition of the 
three behavioral expectancies (i.e., utilitarian, normative, and self-identity expectancies) will better explain the organizational behaviors under investigation. Finally, I argued that affective commitment and the three classes of expectancies will influence the behaviors via attitude toward the behaviors. 


\section{Chapter 5: Method}

In this chapter, I first describe the participants of the current study and the procedure by which the data were collected. Second, I provide detailed description of the measures in the current study including the original measures on which I based to develop the current measures, the modification details for each measure, and other related information about the measures such as their scales and reliabilities.

\section{Participants}

Participants of the current study were recruited from a large private organization in the telecommunication industry in South East of China. There were 330 surveys distributed to the employees at Wave 1 and 296 were received, resulting in a response rate of $89.7 \%$. For Wave 2, 330 surveys were distributed to the employees and the supervisor and 266 were returned, which is a response rate of $80.6 \%$. The matched data from Wave 1 and Wave 2 and between the employees and supervisor resulted in a total sample of 258 participants. The participants were mainly female (73\%) and on average have 15.41 years of education. The average age of the participants was $27.10(S D=2.44)$, and the ages range from 22 to 34 . The average work tenure was $4.21(S D=2.73)$, organizational tenure was $3.37(S D=2.24)$, and job tenure was $3.05(S D=2.11)$. Procedure

The data for the current study were collected in two waves from both employees (measured at both waves) and their supervisors (measured at wave 2). The first wave survey included demographic variables, affective, continuance, and normative commitment, and three classes of behavioral expectancies (i.e., utilitarian, normative and 
self-identity expectancies) measures for each of the behaviors. The questionnaire was sent out together with a cover letter assuring the confidentiality of employees' responses and that the participation is voluntary. The second wave survey was conducted three months later, measuring employees' attitudes toward the behaviors of interest and collecting supervisors' evaluations of each employee in relation to the three behaviors. Measures

Demographic Variables. Employees' tenure, age, education and gender in the organization were obtained from responses to the questionnaires and used as control variables. Early research has shown that age and tenure correlate weakly and positively with all TCM components and gender correlates weakly and negatively with affective and normative commitment (i.e., men tend to have lower affective and normative commitment; Meyer et al., 2002). Therefore, I planned to control for age, gender, education, and tenure in the statistical analyses.

Organizational Commitment. I used the 19-item version of Meyer and Allen's organizational commitment scales (Meyer \& Allen, 1997). Sample items include, affective commitment - "I really feel as if this organization's problems are my own", normative commitment - "I would feel guilty if I left my organization now", and continuance commitment - "Too much of my life would be disrupted if I decided I wanted to leave my organization now." Respondents indicated their agreement with each statement on a 7-point Likert-type scale $(1=$ strongly disagree, $7=$ strongly agree $)$ and their response scores were computed by averaging across the items of each scale. The average reliability of these scales ranges from .76 to .83 (Cooper-Hakim \& Viswesvaran, 
2005). In the current study, the reliabilities for affective, continuance and normative commitment were $.74, .72$ and .82 , respectively. On each sale, higher scores indicated higher commitment.

Considerate Voice. I adapted an eleven-item scale for considerate voice that was developed by Hagedoorn et al. (1999). In the original scale, the targets of measured behaviors were mostly supervisory (e.g., "Try to work out an ideal solution in collaboration with your supervisor"). To assess considerate voice by the organization, in general, I dropped "supervisor" or changed it to "related parties in the organization" (e.g., "Try to work out an ideal solution in collaboration with related parties"). Supervisors indicated their agreement with each statement about each of their subordinates on a 7point Likert-type scale $(1=$ strongly disagree, $7=$ strongly agree $)$ and their response scores were computed by averaging across the items of the scale. Higher scores indicated a higher probability that an individual will engage in the behavior. The reliability of the original scale was .88 in Hagedoorn et al. (1999). In the current study, the reliability for considerate voice scale was .93 .

Production Deviance. I used Spector et al.'s (2006) three-item scale for production deviance. In addition, since the original scale reliability was relatively low (.61), I added two new items to this scale, "Give obscure instructions or information to others" and "Do not provide warning when you know things can go wrong later". Supervisors indicated how often their subordinates engage in the behaviors described in each statement on a 5-point Likert-type scale $(1=$ never, $5=$ everyday $)$ and their response scores were computed by averaging across the items of the scale. Higher scores indicated 
a higher probability that an individual will engage in the behavior. In the current study, the reliability of production deviance scale was .72.

Behavioral Engagement. Currently, to my best knowledge, there is no available scale for behavioral engagement conceptualized following the approach developed by Macey and Schneider (2008). They proposed that behavioral engagement should be broadly defined as adaptive behaviors that are not formally prescribed and go beyond the status quo. Following this conceptualization, I developed my own 8-item behavioral engagement scale for this study. I based my items on Macey and Schneider's (2008) description of behavioral engagement. Sample items are "Willing to take extra responsibility to deal with new organizational challenges" and "Take his/her own risk in initiating new ways of doing things". Supervisors indicated their agreement with each statement about each of their subordinates on a 7-point Likert-type scale $(1=$ strongly disagree, $7=$ strongly agree ) and their response scores were computed by averaging across the items of the scale. Higher scores indicated a higher probability that an individual will engage in the behavior. In the current study, the reliability for behavioral engagement scale was .90 .

Utilitarian Expectancies. Utilitarian expectancies reflect the anticipated rewards or punishments associated with behaviors. In other words, these expectancies are beliefs about whether engaging in the behaviors will yield good result for them. Therefore, I used the phrase "I believe that" followed by the descriptions of the behaviors and ending with either "will yield good results for me" or "will bring good outcomes to me" or simply "will be good for me". Samples items are: "I believe that trying to work out an 
ideal solution in collaboration with related parties will be good for me", for considerate voice; and "I believe that working extra hour to solve organizational problems will bring good outcomes to me", for behavioral engagement. Respondents indicated their agreement with each statement on a 7-point Likert-type scale $(1=$ strongly disagree, $7=$ strongly agree) and their response scores were computed by averaging across the items of the scale. Higher scores indicated a better outcome. In the current study, the reliability for utilitarian expectancies of considerate voice, production deviance, and behavioral engagement scale were $.91, .89$, and .85 , respectively.

Normative Expectancies. Normative expectancies reflect the anticipated approval or disapproval from significant others (e. g., supervisors and coworkers) in the immediate work context. The anticipated approval or disapproval from significant others could be inferred from the individual's evaluation of the frequency that these significant others engage in the same behaviors. Therefore, I used the phrase "People around me at work (e.g., my supervisors and coworkers) usually" followed by the descriptions of the behaviors. Sample items are, "People around me at work (e.g., my supervisors and coworkers) usually try to come to an understanding with parties related to the problem" and "People around me at work (e.g., my supervisors and coworkers) sometimes do their work incorrectly on purpose". Respondents indicated their agreement with each statement on a 7-point Likert-type scale ( 1 = strongly disagree, $7=$ strongly agree $)$ and their responses scores were computed by averaging across the items of the scale. Higher scores indicated a higher approval possibility of the behaviors. In the current study, the 
reliability for normative expectancies of considerate voice, production deviance, and behavioral engagement scale were $.94, .96$, and .91 , respectively.

Self-Identity Expectancies. Self-identity expectancies reflect the anticipated affirmation or repudiation of one's self-concept. In the work context, engaging in a constructive behavior (e.g., considerate voice) could make people feel they are good members (e.g. Organ, 1988) in the organization and engaging in a destructive behavior could make people feel the opposite. Therefore, I used the descriptions of behaviors followed by the phrase "makes me feel as a good member of the organization" for constructive behaviors (i.e., considerate voice and behavioral engagement) or "makes me feel as a bad member of the organization" for destructive behaviors (i.e., production deviance) to measure the self-identity expectancies. Sample items include, "Proactively seeking a better way of doing the job makes me feel as a good member of the organization" (for behavioral engagement) and "Purposely failing to follow instructions makes me feel as a bad member of the organization" (for production deviance). Respondents indicated their agreement with each statement on a 7-point Likert-type scale $(1=$ strongly disagree, $7=$ strongly agree $)$ and their response scores were computed by averaging across the items of the scale. Higher scores indicated a better member of the organization. In the current study, the reliability for self-identity expectancies of considerate voice, production deviance, and behavioral engagement scale were $.93, .99$, and 90 , respectively.

Attitudes toward Behaviors. An attitude toward a behavior is one's evaluation of a specific behavior with some degree of favor or disfavor (Eagly \& Chaiken, 1993). Using 
the semantic differential approach developed by Osgood, Suci, \& Tanenbaum (1957), respondents were asked to indicate their evaluation of each behavior using two bipolar evaluative adjective pairs (i.e., bad-good and harmful-beneficial) on a 7-point Likert-type scale $(1=$ extremely bad/harmful, $7=$ extremely good/beneficial $)$ and their responses scores were computed by averaging across the items of each scale. Higher scores indicated a higher favorability of the behaviors. In the current study, the reliability for attitude toward considerate voice, production deviance, and behavioral engagement scale were $.90, .97$, and .87 , respectively. 
Chapter 6: Results

The analytic procedures and study's results are described in the current chapter. First, I report the results of the scale validation procedures for all the measures of the current study. Second, I present the results of the correlation analyses, which examined the relationships among the study variables. Finally, I provide the results of my regression-based path analysis to test the study's hypotheses. More specifically, in this analysis, the mediation hypotheses are tested directly by testing the indirect effects using bootstrap approach for estimating confidence intervals.

\section{Scale Validation}

Analytical Strategies

The current study investigated the predictive validity of affective commitment, the three classes of behavioral expectancies (i.e., utilitarian, normative and self-identity expectancies), attitude toward behavior (measured by bad-good and harmful-beneficial scale) in predicting several organizational behaviors. The three organizational behavior criteria used to investigate such relationships were considerate voice, production deviance, and behavioral engagement. As described in the method chapter, I have adapted the eleven-item scale for considerate voice developed by Hagedoorn et al. (1999) and the three-item scale for production deviance developed by Spector et al. (2006). Also, I created a new scale for behavioral engagement following the conceptualization developed by Macey and Schneider (2008). In addition, the Macey and Schneider scales were also modified by adding appropriate stems to capture the three classes of behavioral expectancies. As a result, for each organizational behavior criterion, there are five measures (i.e., utilitarian, normative and self-identity expectancies, attitude toward the 
behavior and the behavior itself) that need to be validated to ensure they have construct validity prior to testing the main hypotheses of the study.

Following recommendations by Hinkin (1998), I based on the following analyses to provide validity evidence for the scales developed in the current study: (a) inter-item correlation analysis; (b) exploratory factor analysis (EFA); and (c) confirmatory factor analysis (CFA). In addition, any items flagged for deletion from these quantitative analyses were further examined through item content analysis with theoretical justification to make a final decision on item reduction. In addition, because all the scales involved a particular organizational behavior share the core description describing the behavior of interest, if an item is deleted in one of the scales involved an organizational behavior (e.g., attitude toward production deviance), that item will be removed from all other scales relating to the same behavior (i.e., the three behavioral expectancies of production deviance and production deviance itself), even if it is a good item in the other scales. In the following sections, I will first describe the above analyses and procedures (i.e., inter-item correlations analysis, EFA, and CFA) in more details and then provide the results and conclusion for each of the scales used in the current study.

Inter-item correlations analysis. Inter-item correlations within a variable are indicators of whether the items are drawn from an appropriate domain and it is assumed that all items belong to a common domain should have similar average inter-correlations (Hinkin, 1998). Items with low correlations are considered as sources of error and unreliability (Churchill, 1979). In this analysis, inter-items correlations within each scale 
are examined and items with low correlations (less than .40) with other items are flagged for consideration of deletion.

Exploratory factor analysis. Hinkin (1998) recommended exploratory factor analysis (EFA) is used to refine new scales. EFA is data driven as it imposes no restriction on the pattern of relationship between observed and latent variables (Brown, 2006). EFA helps to derive the smallest number of interpretable factors that can adequately explain the correlations among a set of observed variables. In this analysis, each of the scales is subjected to EFA using Mplus 6.11 (Muthén \& Muthén, 2011) with maximum likelihood estimator and geomin rotation. The number of factors suggested by EFA, which determined by identifying factors with Eigenvalues greater than 1, and the items' factor loadings are examined. "Bad" items, which usually have low inter-item correlations and low factor loadings, will be deleted one by one in this step and an EFA for the remaining items will be conducted until a factor model with clear factor loadings (i.e. factor loadings greater than .40 with no cross-loading items) is achieved. In addition, because each individual scale is supposed to measure a theoretically unidimensional construct, scales with more than one factor as indicated by EFA are also considered to be modified into a single factor model. It is important to note that theoretical justification and item content analysis will always be used in making decision on removing any of the original items. EFA will also be used to investigate whether the three classes of behavioral expectancies, the attitude toward the behavior, and the behavior itself are empirically distinguishable constructs. Information from EFA will provide evidence on the whether the modifications of the items to capture the behavioral expectancies work. 
Confirmatory factor analysis. The purpose of confirmatory factor analysis (CFA) is to verify a factor structure of a set of observed variables. In the other word, CFA is a theory driven technique used to determine if a pre-specified factor solution fit the data well or not. The adequacy of model fit to the data is evaluated through multiple fit statistics such as Chi-square, Comparative Fit Index (CFI), Root Mean Square Error of Approximation (RMSEA), and Standardized Root Mean Square Residual (SRMR). It is important, however, to note that although chi-square statistics is always reported as one of the fit statistics, it is not considered as a useful index because it is affected by sample size and model size. Models with large sample size or large number of variables usually have significant chi-square. A model is considered to have acceptable fit if the CFI is above .90, RMSEA is below .06 and SRMR is below .08 (Hu \& Bentler, 1999). Another important note with regard to model fit statistics is that, while still reported, I did not take RMSEA into account in evaluating the model fit for the individual scales with small model size because there is a greater sampling error for small degree of freedom (df) models. Kenny, Kaniskan, and McCoach (2011) argued that models with small df can have artificially inflated RMSEAs. They suggested that RMSEA should not be even computed for low df models. In this analysis, the single factor structure of the individual scales derived from the previous steps (i.e., inter-item correlation and EFA) and the factor structure of the model that is comprised of all five scales involved each behavior will be evaluated. Besides fit statistics, modification indices for the models that include all five scales will be examined to identify items that generate large residual covariance with other items, which will be further removed from the scales. 


\section{Considerate voice}

Utilitarian expectancies of considerate voice (UECV). Examination of inter-item correlations among eleven items of UECV indicated that all the items were significantly related to one another with most of the correlations ranged between .50 and .70 (see Table 1). However, the correlations of item 5 and 11 with the rest of the items appeared to be relatively smaller. More specifically, almost all item 5's correlations with the other items were in the .30s or low .40s and most of item 11's correlations with the other items were in the $.40 \mathrm{~s}$. Therefore, item 5 and item 11 were noted for further consideration in subsequent analyses. An EFA was conducted on the eleven items of UECV, and the results suggested a one-factor model. Most of the factor loadings were above .70 , except for item 5 with the factor loading of .46 and item 11 with the factor loading of .60 . Examination of the content of item 5 and 11 indicated that both items refer to the use of "compromise" in dealing with organization problems. The low inter-item correlations and relatively low factor loadings of these items suggested that respondents in this particular sample may not have seen compromising as a suitable solution to organizational problems and may have rated them differently compared with the other items in the same scale. Item 5 and 11 were then subsequently removed from the scale one by one. The factor loading matrix for the final solution is presented in Table 2. Two CFAs were conducted to verify the factor structure of the one-factor model for UECV with and without item 5 and 11. As reported in Table 11, the results indicated that the one-factor model for UECV without item 5 and 11 had an acceptable fit, $\chi^{2}(27, N=257)=172.00$, $p<.01, \mathrm{CFI}=.92, \mathrm{RMSEA}=.15, \mathrm{SRMR}=.04$. It also fitted the data significantly better 
than the one-factor model with all the items, $\Delta \chi^{2}(17, N=257)=47.86, p<.01$, which provided further support for the deletion of item 5 and 11.

Normative expectancies of considerate voice (NECV). Table 3 provides the interitem correlations for NECV. As can be seen, most of the correlations were between .60 and .80, except for those involved item 5 and 11, many of which were in the .10 and .30 range. In short, the results indicated that item 5 and 11 were problematic. An EFA was conducted on the eleven items of NECV, and the results suggested a two-factor model with items 1-4 loaded on the first factor and items 5-11 loaded on the second factor. It is also important to note that item 5 had a low factor loading of .30 and item 6 cross loaded on both factors with similar factor loadings (.34 and .47). As described previously, item 5 might not fit with the common content domain on which considerate voice was mapped, and therefore was removed from NECV. Another EFA was run on the remaining 10 items, and the results indicated a one-factor model with most of the factor loadings were above .78 , except for item 11 with a factor loading of .46 . Item 11 was then removed, and the final EFA solution (see Table 4) indicated a one-factor model for NECV. Finally, two CFAs were conducted for the one-factor model of NECV with and without item 5 and 11. As reported in Table 11, the results indicated that the one-factor model for NECV without item 5 and 11 had an acceptable fit, $\chi^{2}(27, N=256)=219.93, p<.01, \mathrm{CFI}=$ $.91, \mathrm{RMSEA}=.17, \mathrm{SRMR}=.04$. It also fitted the data significantly better than the onefactor with all the items, $\Delta \chi^{2}(17, N=256)=44.45, p<.01$, which provided further support for the deletion of item 5 and 11. 
Self-identity expectancies of considerate voice (SECV). Inter-item correlations among the eleven items of SECV were all significant, most of which were between 60 and .70, except for those of item 5 and 11 (see Table 5). More specifically, many correlations of these two items were between high $.20 \mathrm{~s}$ and low $.40 \mathrm{~s}$, which indicated that the two items may have been drawn from different content domains. An EFA was conducted on the eleven items of SECV, and the results suggested a two-factor model with all items (except for item 5 and 11) loaded clearly on the first factor with factor loadings greater than .75. On the other hand, item 5 and 11 loaded on their own factor, with factor loadings of .88 and .43 respectively. It is important to note that item 11 also loaded on the first factor with a factor loading of .35. Based on the content of these items (i.e., compromise in dealing with organizational problems) and the results of the quantitative analyses, item 5 and 11 were then removed one by one from the scale, and the final EFA solution indicated a one-factor model with factor loadings were equal or greater than .80 (see Table 6). Subsequent CFAs for the one-factor model of SECV with and without item 5 and 11 confirmed that the one-factor model without item 5 and 11 had an acceptable fit, $\chi^{2}(27, N=255)=215.99, p<.01, \mathrm{CFI}=.92, \mathrm{RMSEA}=.17, \mathrm{SRMR}=$ .04. It also fitted the data significantly better than the one-factor model with all the items, $\Delta \chi^{2}(17, N=255)=79.77, p<.01($ see Table 11$)$.

Attitude toward considerate voice (ATTCV). Attitude toward considerate voice were measured from two perspectives: (a) whether the behavior is bad or good and (b) whether the behavior is harmful or beneficial. As can be seen in Table 7, most of the inter-item correlations among the 22 items of ATTCV were in the $.50 \mathrm{~s}$ and $.60 \mathrm{~s}$, except 
for those involved item 5 and 11. Correlations of item 5 and 11 were relatively smaller, many of which were in the .20 s and .30 s, suggesting that these were problematic items. An EFA was conducted on the twenty-two items of ATTCV, and the results suggested a three-factor model. Most of the items (except for item 5 and 11) from bad-good scale clearly loaded on the first factor and most of the items (except for item 5) from harmfulbeneficial scale clearly loaded on the second factor. Item 5 from both scales loaded on the third factor. Finally, item 11 from bad-good scale had similar low factor loadings (i.e., smaller than .30) across the three factors.

Based on the results (i.e., low inter-item correlations and factor loadings) as well as the item content (i.e., using compromise to deal with organizational problems), I decided to drop item 5 and 11 from both scales. The final EFA solution suggested a twofactor model with bad-good items loaded on the first factor and harmful-beneficial items loaded on the second factor. The factor loading matrix for the final solution is presented in Table 8. As reported in Table 11, subsequent CFAs indicated that the one-factor model fitted the data significantly worse than the two-factor model, $\Delta \chi^{2}(1, N=258)=142.74, p$ $<.01$, which fitted the data significantly worse than the two-factor model without item 5 and $11, \Delta \chi^{2}(75, N=258)=314.75, p<.01$.

As discussed earlier, attitude toward behavior scale is supposed to measure a theoretically unidimensional construct (i.e., the overall evaluation of engaging a behavior). The current results, on the other hand, suggested that respondents in the sample seemed to have different attitudes depending on how the questions were framed (i.e., from bad-good scale or harmful-beneficial scale). Attitude toward a behavior is 
defined as an overall evaluation of engaging the behavior in question. However, empirical research suggested that attitude often contains two components: instrumental and affective (Ajzen, 2002). Instrumental component is often assessed by adjective pairs as valuable-invaluable or harmful-beneficial. Affective component is reflected in such scales as pleasant-unpleasant or enjoyable-unenjoyable. Ajzen (2002) suggested that bad- good scale is often capable to capture the overall evaluation of the behavior. Research (e.g., Kraft, Rise, Sutton, \& Roysamb, 2005; Lawton, Conner, \& McEachan, 2009) has shown that the two components of attitude have differential relationships with important organizational and individual outcomes. In addition, a recent meta-analysis by Glasman and Albarracin (2006) has illustrated that the extent to which an overall attitude is consistent with instrumental or affective component depends on the behavior under consideration. Therefore, it is possible that bad-good scale and harmful-beneficial scale may have captured slightly different aspects of attitude toward considerate voice. In the current study, I chose to evaluate attitude based on harmful-beneficial scale because instrumental attitude is more specific and has the potential to provide clearer understanding of the relationship between attitude toward behavior and other constructs (i.e. behavioral expectancies and behaviors).

The final CFA solution (see Table 11) suggested that a one-factor model with only harmful-beneficial items (without item 5 and 11) had an acceptable fit, $\chi^{2}(27, N=$ $258)=127.60, p<.01, \mathrm{CFI}=.94, \mathrm{RMSEA}=.12, \mathrm{SRMR}=.04$. It also fitted better to the data than the two-factor model with items from both scales (without item 5 and 11), $\Delta \chi^{2}$ $(106, N=258)=370.84, p<.01$. 
Considerate voice $(\mathrm{CV})$. Inter-item correlations of $\mathrm{CV}$ were presented in Table 9. Similar to other scales that involved considerate voice, correlations of item 5 and 11 with the other items were relatively smaller than the rest of the correlations. Item 5 was especially troublesome as its correlations were mostly in the .10s and .20s. An EFA was conducted for the eleven items of $\mathrm{CV}$, and the results suggested a two-factor model with items 1-8 loaded on the first factor and items 9-11 loaded on the second factor (see Table 10). Examination of item content for item 9 - "Immediately try to find a solution" - and item 10 - "Try to think of different solutions to the problem" - indicated that they are different from the rest of the items in that they do not specifically mention about "working with other people". Also, the content of the items is also vague to the extent whether employees do or do not take both the individual's concerns and those of the organization into consideration. This distinction might have become noticeable by the supervisors who rated their employees in term of considerate voice behaviors. For that reason, I proceeded with deleting item 9 and 10 from the scale. In addition, item 5 and 11 were also removed from the scale because of the low inter-item correlations with the rest of the items. As presented in Table 11, CFAs indicated the final one-factor model with 7 items (i.e., items $1-4,6,7$, and 8 ) had an acceptable fit, $\chi^{2}(14, N=257)=121.00, p<.01$, $\mathrm{CFI}=.93, \mathrm{RMSEA}=.17, \mathrm{SRMR}=.04$. It also fitted the data significantly better than the two-factor model, $\Delta \chi^{2}(29, N=257)=175.86, p<.01$.

In conclusion, the results indicated that the final scale for the three behavioral expectancies of considerate voice consisted of 9 items (i.e., items 1-4, 6-10). The final scale of attitude toward considerate voice consisted of the same 9 items but were from 
only the harmful-beneficial scale. The reason for excluding bad-good scale from the study is because it tends to capture both components of attitude, which might mask our understanding of the true relationship between each component of attitude toward behavior and the behavior. Finally, the final scale for considerate voice consisted of 7 items (i.e., items 1-4, 6-8). Putting together, there were 7 items (i.e., items 1-4, 6-8) that survived the item reduction procedures for each of the individual scales involved considerate voice.

To test whether the measures involved considerate voice were empirically distinguishable, I conducted an EFA on the 35 items from all the five scales involved considerate voice. The results suggested a five-factor model with items of each scale loaded clearly on the same factor on which they were supposed to load with all factor loadings are above .50 (see Table 12). A CFA was then conducted to test whether the five-factor model has an adequate fit to the data. The results indicated that the model has a good fit to the data, $\chi^{2}(395, N=258)=840.72, p<.01, \mathrm{CFI}=.94, \mathrm{RMSEA}=.07$, $\mathrm{SRMR}=.04$. However, modification indices suggested that item 7 and 6 generated large residual covariance with each other and the other items in most of the scales (e.g., UECV, SECV, and CV). For this reason, I decided to remove item 7 and 6 one by one from all the scales and conducted a CFA for the five-factor model after removing each of the item to test whether the deletion of these items significantly improve the model fit. The results suggested that the model with 5 items in each scale (i.e., without item 6 and 7) fitted the data significantly better than the model with 6 items in each scale (i.e., without item 7; $\left.\Delta \chi^{2}(130, N=258)=239.90, p<.01\right)$, which fitted the data significantly better than the 
model with 7 items in each scale, $\Delta \chi^{2}(155, N=258)=428.47, p<.01$. Therefore, item 6 and 7 were further deleted from all individual scales involved considerate voice. Finally, Cronbach's alphas for all measures were computed and the results indicated that each of these alphas was above the generally accepted .70 cutoff criteria for acceptable internal consistency, which provided support for the internal reliability of these measures (see Table 40). In conclusion, 5 items (i.e., items 1-4, and 8) were retained for each individual scale involved considerate voice.

\section{Production Deviance}

Utilitarian expectancies of production deviance (UEPD). Examinations of interitem correlations of UEPD indicated that all items were highly correlated to one another, most of which were in the $.50-.60$ range. Correlations among UEPD's items are presented in Table 13. An EFA was conducted on the 5 items of UEPD, and the results suggested a one-factor model with factor loadings are above .70, suggesting they were good items. The factor loading matrix for UEPD is displayed in Table 14. A CFA was conducted to investigate whether the one-factor model for UEPD had an adequate fit to the data. As reported in Table 23, the results indicated that it had a good model fit, $\chi^{2}(5$, $N=248)=14.85, p<.05, \mathrm{CFI}=.99, \mathrm{RMSEA}=.09, \mathrm{SRMR}=.02$.

Normative expectancies of production deviance (NEPD). The inter-items correlations of NEPD are presented in Table 15. NEPD's items were highly correlated. All of the correlations were in low .80s suggesting that the items were drawn from the same domain. An EFA was conducted on the 5 items of NEPD, and the results suggested a one-factor model with factor loadings were above .90 (see Table 16). Finally, a CFA 
was conducted to investigate whether the one-factor model for NEPD had an adequate fit to the data. As reported in Table 23, the results indicated that it had a good model fit, $\chi^{2}(5, N=256)=23.42, p<.01, \mathrm{CFI}=.99, \mathrm{RMSEA}=.12, \mathrm{SRMR}=.01$.

Self-identity expectancies of production deviance (SEPD). Similar to utilitarian and normative expectancies of production deviance, SEPD's items were highly correlated with inter-item correlations were all above .90 (see Table 17). An EFA was conducted on the 5 items of SEPD, and the results suggested a one-factor model with factor loadings were above .95 (see Table 18). Finally, a CFA was conducted to investigate whether the one-factor model for SEPD had an adequate fit to the data. As reported in Table 23, the results indicated that it had a good model fit $\chi^{2}(5, N=252)=60.12, p<.01, \mathrm{CFI}=.98$, $\mathrm{RMSEA}=.21, \mathrm{SRMR}=.01$.

Attitude toward production deviance (ATTPD). Inter-item correlations among ATTPD's items suggested that correlations between items within bad-good or harmfulbeneficial scale tended to correlate more strongly than those between items across the scales (see Table 19). However, the differences were rather small, about .10. An EFA was conducted on the 10 items of ATTPD, and the results indicated a one-factor model with factor loadings were above .70. Similar to the approach for attitude toward considerate voice, I decided to use only items from harmful-beneficial scale to assess attitude toward production deviance. Another EFA was conducted on only harmfulbeneficial items, and the results suggested a one-factor model (see Table 20). Most of the loadings were above .90 , except for item 5 with a factor loading of .75 . A CFA was conducted to test whether the one-factor model with the five items from harmful- 
beneficial scale had adequate model fit. As reported in Table 23, the result indicated that the model had a good model fit, $\chi^{2}(5, N=258)=22.05, p<.01, \mathrm{CFI}=.99$, RMSEA $=$ $.12, \mathrm{SRMR}=.02$.

Production Deviance (PD). Inter-item correlations for PD are presented in Table 21. Although most of the correlations were above .35 , correlations of item 5 were very small, mostly in .10s. In fact, the correlation between item 2 and 5 was not statistically significant $(r=.10)$. An EFA was conducted on the 5 items of PD, and the results suggested a one-factor model with most of the factor loadings were above .50 , except for item 5 with a factor loading of .23 (see Table 22). Further examination of the item 5 content - "Do not provide warning when you know things can go wrong later" - suggests that it might have been difficult for the supervisors to have a good observation of this behavior because it is usually known only by employees. This might have been the reason for item 5 to behave differently in the PD scale. For this reason, item 5 was deleted from the scale. Finally, a CFA was conducted for the one-factor model of PD with items 1-4, and the results indicated that the model had a good model fit, $\chi^{2}(2, N=$ $257)=8.04, p<.01, \mathrm{CFI}=.98, \mathrm{RMSEA}=.11, \mathrm{SRMR}=.03($ see Table 23$)$.

In summary, the results indicated that the final scale for the three behavioral expectancies of production deviance consisted of 5 items. The final scale for attitude toward production deviance also consisted of the same 5 items but only from harmfulbeneficial scale. I chose to use only items from the harmful-beneficial scale for attitude toward production deviance in order to be consistent with the approach used for attitude toward considerate voice. Finally, the final scale for production deviance consisted of 
only 4 items. As discussed, the difficulty for supervisor to be able to observe and rate this behavior may have led to the low inter-item correlations as well as low factor loading for item 5. Taken together, I decided to drop item 5 from all the scales involved production deviance.

To test whether the measures involved production deviance were empirically distinguishable, I conducted an EFA on the 20 items from all the five scales involved production deviance. The results suggested a five-factor model with items of each scale loaded clearly on the same factor on which they were supposed to load (see Table 24). A CFA was then conducted to test whether the five-factor model had an adequate fit to the data. The results indicated that the model had a good fit to the data, $\chi^{2}(160, N=258)=$ $278.54, p<.01, \mathrm{CFI}=.98, \mathrm{RMSEA}=.05, \mathrm{SRMR}=.04$. Modification indices did not suggest any item that generated large residual covariance with other items. Finally, Cronbach's alpha for each of the measures was computed and the results indicated that each of these alphas was above the generally accepted .70 cutoff criteria for acceptable internal consistency, which provide support for the internal reliability of these measures (see Table 40). In conclusion, 4 items (i.e., items 1-4) were retained for each individual scale involved production deviance.

\section{Behavioral Engagement}

Utilitarian expectancies of behavioral engagement (UEBE). Table 25 presents inter-item correlations among UEBE's items. While all the correlations were significant and greater than .30 , the pattern of correlations suggested that the items were clustered into two groups, with the first group consisted of items 1-4 and the second group 
consisted of items 5-8. Descriptively, the correlations within each group were generally larger than those between groups. Subsequent EFA on the eight items suggested a twofactor model with items 1-4 loaded clearly on the first factor and items 5-8 loaded clearly on the second factor. All factor loadings were .50 and above suggesting they were good items (see Table 26). Examination of the items' content suggested that the two groups might have captured different constructs. More specifically, what shared by items 1-4 is that they all refer to doing extra things (e.g., working extra hour or doing extra work) for the organization. On the other hand, items 5-8 are qualitatively different as they refer to initiative behaviors (e.g., seeking a better way of doing the job), risk-taking behaviors (e.g., taking risk to initiate new way of doing things), or adaptive behaviors (e.g., monitoring the environment for relevant changes). Although Macey and Schneider (2008) argued that extra effort (i.e., doing something more) is a facet of behavioral engagement, they also recognized that engagement should be defined more broadly (see proposition 7; Macey \& Schneider, 2008) to include initiative, proactive, and adaptive behaviors. The empirical evidence in the current study suggested that employees do recognize the difference between doing something more and doing something different. For this reason, I decided to drop items 1-4 from the current scale because items 5-8 appear to capture a greater criterion domain of behavioral engagement. A CFA was conducted to evaluate the one-factor model with items 5-8. As reported in Table 35, the results indicated that the one-factor model fit the data extremely well, $\chi^{2}(2, N=256)=$ $1.78, p>.05, \mathrm{CFI}=1.00, \mathrm{RMSEA}=0, \mathrm{SRMR}=.008)$ 
Normative expectancies of behavioral engagement (NEBE). Inter-item correlations among NEBE's items are presented in Table 27. Similar to UEBE, the pattern of the correlations suggests that there were two groups of items with items 1-4 in one group and items 5-8 in the other group. Correlations of items between groups were generally smaller than those within each group. I conducted an EFA on the eight items of NEBE, and the results suggested a two-factor model with items 1-4 loaded on the first factor and items 5-8 loaded on the second factor (see Table 28). Most of the factor loadings were greater than .50 except for item 4 with the factor loading of .46 . Item 4 also cross-loaded on the second factor with a factor loading of .42. As discussed earlier, the difference in the items' content might be attributable to the difference between the two groups of the items in that items 1-4 refer to doing something more for the organization, whereas items 5-8 refer to doing something different for the organization. Therefore, I decided to drop items 1-4 from the NEBE scale and conducted a CFA for the one-factor model with items 5-8. The results indicated that the one-factor model had an excellent fit, $\chi^{2}(2, N=255)=.55, p>.05, \mathrm{CFI}=1.00, \mathrm{RMSEA}=0, \mathrm{SRMR}=.004($ see Table 35$)$. Self-identity expectancies of behavioral engagement (SEBE). Examination of inter-item correlations among SEBE's items indicated that item 3 was problematic (see Table 29). More specifically, while most of the correlations were in the .50s and $.60 \mathrm{~s}$, item 3 correlated relatively less strongly with the rest of the items ( $r$ s are in the .10 s and .20 s). An EFA was conducted on the 8 items of SEBE, and the results suggested a onefactor model. Item 3's factor loading was .27 while others' loadings were greater than .70 (see Table 30). It was also intriguing to note that except for item 3, all items were loaded 
clearly on one factor, which is very different from the results of UEBE and NEBE. However, I think the results actually fit with the nature of the self-identity expectancies of behavioral engagement. More specifically, while employees recognize the differences in the benefits (utilitarian expectancies) and expectations of others (normative expectancies) between doing some more and doing something different, both types of behaviors can make them feel as a good member of the organization. Two CFAs were conducted on the one factor model with and without item 3, and the results indicated that the one-factor model without item 3 did not fit the data significantly better than the onefactor with all the items. Both of the models did not reach an acceptable level of fit (see Table 35). Due to the lack of fit to the data, I examined the modification indices, and found that correlating error coefficients of item 1 and 2 would improve the model fit. Both of items 1 and 2 are similar in that they refer to "working extra hour" which suggested that they may share some error variance simply due to the same phrase. As reported in Table 35, the updated model fitted the data significantly better than the onefactor model without item $3, \Delta \chi^{2}(1, N=254)=62.44, p<.01$, and the model also had improved its fit statistics, $\chi^{2}(13, N=254)=90.48, p<.01, \mathrm{CFI}=.94, \mathrm{RMSEA}=.15$, $\operatorname{SRMR}=.04$.

Attitude toward behavioral engagement (ATTBE). Inter-item correlations for ATTBE are presented in Table 31. Descriptively, all correlations were significant and majority of them were in the $.40 \mathrm{~s}$ and .50 s with no unusual pattern. An EFA was conducted on the 16 items from both bad-good scale and harmful-beneficial scale. The results suggested a two-factor model with items 1-3 from both scales loaded clearly on 
the first factor and items 4, 5, 7 and 8 loaded clearly on the second factor (see Table 32). In addition, item 6 from both scales cross-loaded on both factors. The results for ATTBE were similar to those of UEBE and NEBE, except for item 4 was now loaded on the second factor with items 5-8. It is unclear why item 4 behaved differently in ATTBE, except for a subtle difference in the item 4's content compared with items 1-3's content. More specifically, item 4 refers to "taking extra responsibility" for "new organizational challenge" while items 1-3 refer simply to "working extra hour" or " doing extra work" for "additional organizational demand" or "solving organizational problems". One can argue that taking extra responsibility is similar to vertical job enlargement, rather than horizontal job enlargement, which would require more skills and efforts. In addition, an aspect of the survey administration that may have made this subtle difference noticeable to the respondents is the fact that employees took the survey on attitude toward behavioral engagement after taking the survey on the three behavioral expectancies with the same items three times. Familiarity with the items' content might have helped the respondents to distinguish item 4 from items 1-3. Because of the EFA results as well as the difference in the items' content between item 4 and items 1-3, I decided to remove items 1-3 from both scales (i.e., bad-good and harmful-beneficial) and did another EFA on the remaining 10 items. The result indicated a one-factor model with only one Eigenvalues greater than 1. However, as discussed in the EFA/CFA for attitude toward considerate voice, I decided to drop all items from bad-good scale to make sure I evaluate attitude toward behavior consistently across the three organizational behaviors. A CFA was conducted on the one-factor model with item 4-8, and the results indicated that the 
model had a good fit to the data, $\chi^{2}(33, N=258)=216.57, p<.01, \mathrm{CFI}=.92, \mathrm{RMSEA}=$ $.15, \mathrm{SRMR}=.04($ see Table 35$)$.

Behavioral Engagement (BE). The inter-item correlations of $\mathrm{BE}$ are presented in Table 33. Descriptively, most of the correlations were above .50, suggesting items were similar and highly correlated to one another. An EFA was conducted on the 8 items of BE, and the results suggested a two-factor model with items 1-3 loaded on the first factor and item 4-8 loaded on the second factor (see Table 34). All factor loadings were equal or above .70 suggesting they were good items. Similar to attitude toward behavioral engagement, the subtle difference between item 4 and items 1-3, which has been discussed earlier, might be the reason for item 4 loaded on the second factor with items 58. It suggested that the supervisors did recognize the difference between taking new responsibilities and just simply doing something more. I therefore proceeded with deleting items 1-3 from the BE scale. A CFA was conducted for the one-factor model with item 4-8, and the results indicated a good model fit, $\chi^{2}(5, N=257)=74.17, p<.01$, $\mathrm{CFI}=.93, \mathrm{RMSEA}=.23, \mathrm{SRMR}=.04($ see Table 35$)$.

In summary, the results suggested that the final scale for utilitarian and normative expectancies of behavioral engagement consisted of items 5-8. For self-identity expectancies, the final model consisted of item 1,2, and item 4-8. The attitude toward behavioral engagement had its final scale consisted of item 4-8 and these items were only from the harmful-beneficial scale. Finally, behavioral engagement consisted of item 4-8. As discussed earlier, if an item was deleted in one scale, the same item would be deleted 
in all other scales involved the same behavior, I therefore deleted items 1-4 from all the individual scales involved behavioral engagement.

To test whether the measures involved behavioral engagement were empirically distinguishable, I conducted an EFA on the 20 items from all the five scales involved behavioral engagement. The results suggested a five-factor model with items of each scale loaded clearly on the same factor on which they were supposed to load (see Table 36). A CFA was then conducted to test whether the five-factor model has an adequate fit to the data. Results indicated that the model had a good fit to the data, $\chi^{2}(160, N=258)=$ $314.58, p<.01, \mathrm{CFI}=.96, \mathrm{RMSEA}=.06, \mathrm{SRMR}=.04$. Modification indices did not suggest any item that generates large residual covariance with other items. Finally, Cronbach's alpha for each of the measures was computed and the results indicated that each of these alphas was above the generally accepted .70 cutoff criteria for acceptable internal consistency, which provided support for the internal reliability of these measures (see Table 40). In conclusion, 4 items (i.e., items 5-8) were retained for each individual scale involved behavioral engagement.

\section{Measurement Model Testing}

After conducting item reduction and providing evidence of construct validity for the 15 scales involved the three organizational behaviors, I conducted a series of CFA to test the overall measurement model with an addition of organizational commitment components (i.e., affective, continuance, and normative commitment). More specifically, CFAs were conducted to investigate the factor structure the measurement model that included all latent constructs (i.e., three components of organizational commitment, three 
classes of behavioral expectancies, attitude toward the behavior and the behavior itself) for each of the behaviors in the study. The results of these CFAs are presented in Table 37. In short, the results suggested that the eight-factor model for each of the behaviors did not fit the data.

Since the models without organizational commitment components have shown good model fits, the reasons for the lack of fit to the data of the overall measurement model were more likely come from Meyer and Allen's organizational commitment scale. I conducted a CFA for a three-factor model of organization commitment to investigate where the problem was. The results indicated that the three-factor model did not have an acceptable fit to the data, $\chi^{2}(149, N=258)=765.30, p<.01, \mathrm{CFI}=.68, \mathrm{RMSEA}=.13$, SRMR =.14). Because the CFA indicated that organizational commitment scale did not fit the data, I decided to run an EFA to investigate the factor structure of the scale with the current study's sample. As reported in Table 38, the EFA results suggested a fourfactor model with all the items from continuance commitment scale loaded on one factor, item 3 and 4 from affective commitment scale loaded on another factor, item 2 and 3 from normative commitment scale yet loaded on another separated factor, and item 1, 2, 5 from affective commitment scaled loaded on the same factor with item 4, 5, and 6 from normative commitment scale. In addition, item 6 from affective commitment scale and item 1 from normative commitment scale appeared not to be loaded on any of the four factors. Finally, item 3 from continuance commitment scale was also cross-loaded on the same factor with several items from affective and normative commitment scale. In other 
words, the results suggested that there were problems with each component of the organizational commitment scale.

To further investigate the issue, I did three EFAs separately for the three components of the organizational commitment scale. The results are displayed on Table 39. For affective commitment, the results indicated a two-factor model with item 1,2 and 5 loaded on the first factor, and item 3 and 4 loaded on the second factor. In addition, item 6 was crossed-loaded on both factors. For normative commitment, the result indicated a two-factor model with item 4-6 loaded on the first factor and item 2 and 3 loaded on the second factor. Item 1 appeared to be a bad item, as it did not load on either factor. For continuance commitment, the results indicated a two-factor model with item 1, 2 and 3 loaded on the first factor and items 4-7 loaded on the second factor. Although loaded on the second factor, item 4 was also cross-loaded on the first factor with a smaller factor loading (.33 in factor 1 vs. .52 in factor 2). In summary, the EFA results suggested that the following items were problematic: Item 3, 4 and 6 from affective commitment scale, item 1 from normative commitment scale, and the split within continuance commitment scale between items 1-3 and items 4-7.

For normative commitment, I first removed item 1 from the normative commitment scale. It is important to note that item 1 - "I do not feel a strong sense of belonging to my organization" - is the only negatively worded item in the scale, and it may have had a different meaning for the respondents. Another EFA was conducted for the rest of the items and the result suggested a one-factor model with factor loadings were above 4.7. For this reason, I decided to keep the rest of the items in the normative 
commitment scale. For affective commitment, it is intriguing to note that all the problematic items such as item 3 - "I do not feel like "part of the family" at my organization", item 4 - "I do not feel "emotionally attached" to this organization", and item 6 - "I do not feel a strong sense of belonging to my organization" are also negatively worded items. Therefore, it is possible that the negatively worded items might have captured a different construct compared with the positively worded items in this particular sample. Although negatively worded items are argued to be able to reduce response biases (Price \& Mueller, 1986), others have found that these items can have a detrimental effect on the psychometric properties of a measure (Harrison \& McLaughlin, 1991). For this reason, I decided to remove these items (i.e., item 3, 4, and 6) from the affective commitment scale and use only item 1,2 and 5 for the current study.

For continuance commitment, it is possible that the problem was caused by the sub-dimension issue that has been mentioned frequently in the TCM literature (Solinger et al., 2008). More specifically, continuance commitment scale is comprised of two subdimensions: lack of alternatives and high sacrifices. McGee and Ford (1987) argued that the two sub-dimensions often operate in an opposite direction which may create problem for the overall continuance commitment scale. Many of the previous studies attempted to evaluate the dimensionality of continuance commitment scale also have found support for the two-factor structure (e.g., Hackett, Bycio, \& Hausdorf, 1994; Meyer, Allen, \& Gellatly, 1990; Somers, 1993). Examination of items' content indicated that item 1-5 are “alternative" items (i.e. reflecting a perceived lack of alternative employment opportunities) and item 6 and 7 are "sacrifices" items (i.e. reflecting perceived sacrifices 
associated with leaving the organization). Because item 4 was cross-loaded on both factors and item 5 was not loaded on the factor on which it is supposed to load (i.e., alternatives), I decided to remove them from the continuance commitment scale. With regard to the two sub-factor issue of continuance commitment scale, I decided to use only item 1-3 for the current study to retain a maximum amount of items from the original scale. However, the fact that I use only a sub-dimension of the scale and that the two subdimensions were only moderately related to one another $(r=.27, p<.01)$ will have implications on the results of the current study.

In summary, the results of factor analysis suggest that organizational commitment scale did not function as expected. Further investigation suggested that all the negatively worded items (i.e., item 3, 4, and 6 from affective commitment scale and item 1 from normative commitment scale) were problematic and appeared to not have captured their targeted constructs successfully. For this reason, I decided to remove all the negatively worded items from the organizational commitment scale for the current study. The new affective commitment scale now consists of item 1,2 and 5 and the new normative commitment scale is now comprised of item 2-6. In addition, the current study also encountered the dimensionality issue of the continuance commitment scale. More specifically, the results suggested a two-factor model (i.e., lack of alternatives and high sacrifices) for continuance commitment. I decided to use only item 1-3 from the "lack of alternatives" scale for testing the hypotheses of the study.

With a new set of organizational commitment items, I conducted another CFA to investigate a three-factor model for organizational commitment scale. The results 
suggested the three-factor model did not fit the data sufficiently, $\chi^{2}(41, N=258)=$ $180.16, p<.01, \mathrm{CFI}=.88, \mathrm{RMSEA}=.12, \mathrm{SRMR}=.07$. Because of this, $\mathrm{I}$ decided to examine the modification indices and found that the model fit could improve significantly if I correlated the error coefficients of item 2 and 3 from the normative commitment scale, $\Delta \chi^{2}(1, N=258)=43.44, p<.01$. In general, the practice of correlating error terms is considered acceptable if there is a reason to believe that the two items share some error variance together. Item 2 - "Even if it were to my advantage, I do not feel it would be right to leave my organization now" and item 3 - "I would feel guilty if I left my organization now" are quite similar in that "feeling guilty" may exert a similar effect as does "feeling not right" on the respondents, and the two items might share some variance just because of that. The updated model also had an acceptable fit, $\chi^{2}(40, N=258)=$ $136.72, p<.01, \mathrm{CFI}=.92, \mathrm{RMSEA}<.10, \mathrm{SRMR}=.07$.

Finally, another series of CFA was conducted to test the overall measurement model with the newly modified organizational commitment scale. The results indicated that, for each of the three organizational behaviors, the eight-factor model with the new organizational commitment scale fitted the data significantly better than the same eightfactor model with the original organizational commitment scale. The results also indicated the overall measurement models for the three organizational behaviors had good model fit to the data. For more details of the models' fit statistics, please refer to Table 37. 


\section{Correlation Analysis}

Means, standard deviations, and intercorrelations of among study's variables are displayed in Table 43. Among demographic variables, age was not correlated with any variables included in the study except for organizational tenure $(r=.80, p<.01)$. Gender had a positive relationship with education $(r=.21, p<.01)$ and a negative relationship with organizational tenure $(r=-.25, p<.01)$, which indicated that men tend to have more year of education than women but spend less time with the organization. In addition, gender had a positive relationship with attitude toward considerate voice $(r=.14, p<$ $.05)$, attitude toward behavioral engagement $(r=.16, p<.05)$, and production deviance $(r$ $=.16, p<.01)$. Besides having a positive correlation with gender, education was negatively related to organizational tenure $(r=-.29, p<.01)$, indicating that people with

a more year of education tend to spend less time with the organization. Finally, except for the reported relationships with age, gender, and education, organizational tenure was not related to any other variables in the current study. In short, the observed relationship suggested that gender was related to some key variables in the study, and therefore will be used as a control variable in all analyses testing the study's hypotheses.

Among organizational commitment components, affective commitment was positively and highly related to normative commitment $(r=.69, p<.01)$. Continuance commitment was positively related to both affective and normative commitment with essentially the same magnitude $(r=.38, p<.01)$. As mentioned, continuance and normative commitment will join gender as control variables for all the analyses of the study. With regard to other key variables of the study, I found that affective, continuance 
and normative commitment correlated with the three behavioral expectancies, attitude toward behavior and the behavior itself in a very similar fashion, except for the strengths of their correlations. In general, correlations involved affective commitment were larger than correlations involved normative commitment, which were larger than correlations involved continuance commitment. More specifically, affective commitment correlated positively with all three behavioral expectancies of considerate voice $(r=.53, p<.01$ for utilitarian expectancies; $r=.51, p<.01$ for normative expectancies; and $r=.45, p<.01$ for self-identity expectancies) and behavioral engagement $(r=.27, p<.01$ for utilitarian expectancies; $r=.42, p<.01$ for normative expectancies; and $r=.41, p<.01$ for selfidentity expectancies), but negatively related to utilitarian and normative expectancies of production deviance with the same magnitude $(r=-.45, p<.01)$. Normative commitment was positively related to all three behavioral expectancies of considerate voice $(r=.46, p$ $<.01$ for utilitarian expectancies; $r=.44, p<.01$ for normative expectancies; and $r=.43$, $p<.01$ for self-identity expectancies) and behavioral engagement $(r=.22, p<.01$ for utilitarian expectancies; $r=.43, p<.01$ for normative expectancies; and $r=.32, p<.01$ for self-identity expectancies), but negatively related to utilitarian $(r=-.26, p<.01)$ and normative expectancies $(r=-.33, p<.01)$ of production deviance. Finally, continuance commitment was correlated positively with all three behavioral expectancies of considerate voice $(r=.24, p<.01$ for utilitarian expectancies; $r=.24, p<.01$ for normative expectancies; and $r=.25, p<.01$ for self-identity expectancies) and behavioral engagement $(r=.20, p<.01$ for utilitarian expectancies; $r=.20, p<.01$ for normative expectancies; and $r=.23, p<.01$ for self-identity expectancies), but 
negatively related to utilitarian $(r=-.14, p<.05)$ and normative expectancies $(r=-.13, p$ $<.05)$ of production deviance.

With regard to attitudes toward behaviors, both affective and normative commitment had significant correlations with attitude toward considerate voice and attitude toward behavioral engagement but not with attitude toward production deviance. More specifically, affective commitment was positively correlated with attitude toward considerate voice $(r=.25, p<.01)$ and attitude toward behavioral engagement $(r=.24, p$ $<.01)$. Similarly, normative commitment was positively correlated with attitudes toward considerate voice $(r=.17, p<.01)$ and attitude toward behavioral engagement $(r=.21, p$ $<.01)$. On the other hand, continuance commitment was only related to attitude toward behavioral engagement $(r=.16, p<.01)$. Finally, with regard to the behaviors, none of the components of organizational commitment was related to any of the organizational behaviors with correlations mostly around .10 .

Among variables involving considerate voice, behavioral expectancies were highly related to one another. Utilitarian expectancies was positively correlated with normative expectancies $(r=.71, p<.01)$ and self-identity expectancies $(r=.68, p<.01)$, and normative expectancies was positively related to self-identity expectancies $(r=.74, p$ $<.01)$. In addition, attitude toward considerate voice correlated positively with the three behavioral expectancies of considerate voice $(r=.35, p<.01$ for utilitarian expectancies; $r=.34, p<.01$ for normative expectancies; and $r=.37, p<.01$ for self-identity expectancies). Finally and contradicted to my expectation, considerate voice was not 
significantly related to attitude toward considerate voice nor any of its behavioral expectancies with correlations ranging from .07 to .12 .

Among variables involving behavioral engagement, behavioral expectancies were highly related to one another. More specifically, utilitarian expectancies was positively related to normative expectancies $(r=.49, p<.01)$ and self-identity expectancies $(r=$ $.53, p<.01)$. Normative expectancies was positively related to self-identity expectancies $(r=.55, p<.01)$. In addition, attitude toward behavioral engagement correlated positively with the three behavioral expectancies of behavioral engagement $(r=.35, p<$ .01 for utilitarian expectancies; $r=.31, p<.01$ for normative expectancies; and $r=.31, p$ $<.01$ for self-identity expectancies). Finally, behavioral engagement was only significantly correlated to attitude toward behavioral engagement $(r=.23, p<.01)$ and its normative expectancies $(r=.14, p<.05)$ but not its utilitarian and self-identity expectancies.

With regard to production deviance, the pattern of correlation among related variables did not resemble to those of considerate voice and behavioral engagement. More specifically, while utilitarian and normative expectancies of production deviance were highly related to each other $(r=.52, p<.01)$, none of them was related to selfidentity expectancies of production deviance. On the other hand, only self-identity expectancies was related to attitude toward production deviance $(r=-.15, p<.01)$. Finally, except for a positive correlation with attitude toward production deviance $(r=$ $.22, p<.01)$, production deviance was not significantly related to any of the three behavioral expectancies with correlations ranging from -.03 to .12 . 
Finally, with regard to the relationships across the three organizational behaviors, I found that considerate voice was positively related to behavioral engagement $(r=.51, p$ $<.01)$ while production deviance was negatively related to both considerate voice $(r=-$ $.34, p<.01)$ and behavioral engagement $(r=-.33, p<.01)$.

\section{Structural Model Testing}

\section{Path Analyses}

Before testing the structural models for each of the behaviors, I tested the measurement models $\left(\mathrm{M}_{0}\right)$ again with an addition of an observed covariate (i.e., gender). The covariate was included to ensure that the structure equation models of interest, in which gender is used as one of the control variables, were nested to the corresponding measurement models. The latent constructs in the measurement model were regressed on gender. The results indicated that the 8-factor model for each behavior had a good fit to the data. For considerate voice, $\chi^{2}(594, \mathrm{~N}=255)=1100.27, p<.01, \mathrm{CFI}=.92$, RMSEA $=.06, \mathrm{SRMR}=.05$; for production deviance, $\chi^{2}(429, \mathrm{~N}=255)=762.38, p<.01, \mathrm{CFI}=$ $.95, \mathrm{RMSEA}=.06, \mathrm{SRMR}=.05$; for behavioral engagement, $\chi^{2}(429, \mathrm{~N}=255)=851.30$, $p<.01, \mathrm{CFI}=.91, \mathrm{RMSEA}=.06, \mathrm{SRMR}=.05$. Therefore, the measurement models appeared to be well-suited for testing the structural models.

The following sections will detail the procedures that I followed to test the hypotheses of the current study. In general, for each of the organizational behavior, the full mediation model $\left(\mathrm{M}_{1}\right)$ and the partial mediation model $\left(\mathrm{M}_{2}\right)$ were tested on the basis of the measurement model $\left(\mathrm{M}_{0}\right)$. Figure 1 illustrates the structural paths in $\mathrm{M}_{1}$. Note that none of the direct effects of the affective commitment and the three classes of expectancies were estimated on the behaviors. To test $\mathrm{M}_{2}$, all the direct paths omitted 
from M1 were added to the structural model (see Figure 2). In both $\mathrm{M}_{1}$ and $\mathrm{M}_{2}$, gender, continuance commitment, and normative commitment were used as control variables simultaneously for both the mediator (attitudes toward the behaviors) and the outcome (the behaviors). In addition, given that my hypotheses are on the role of attitude toward the behavior as the mediator of the model, I also tested another alternative model $\left(\mathrm{M}_{3}\right)$ with attitude toward the behavior serves as a regular antecedent in the model. In other words, I set the paths between affective commitment and the three classes of behavioral expectancies and attitude toward the behavior to zero (Figure 3). The decision on which model to use for hypothesis testing were made based on the fit indices and the differences between these models.

To test the study's hypotheses, I followed the following steps: (1) entering the control variables in the model with the mediator and the outcome; (2) adding affective commitment into the model; (3) adding the three behavioral expectancies into the model; (4) adding the indirect effects; and (5) testing the indirect effects using bootstrap approach. Bootstrapping is a non-parametric technique for effect size estimation and hypothesis testing that makes no assumptions about the shape of the distributions of the variables or the sampling distribution of the statistic (Preacher \& Hayes, 2004). The method is considered as a preferable approach for testing the indirect effect because of its advantage in overcoming the problem of nonnormality in the sampling distribution of the indirect effect as well as its ability to produce test with small samples (MacKinnon, Lockwood, \& Williams, 2004; Preacher \& Hayes, 2004). In addition to the estimate of the indirect effect and its standard error, bootstrap also provides both $95 \%$ and $99 \%$ 
confidence intervals for the population value of the indirect effect, which provide information on whether the indirect effect is significantly different from zero. For example, if zero is not in the $95 \%$ confidence interval, we can conclude that the indirect effect is significantly different from zero at $p<.05$. In the current study, $95 \%$ percentile bootstrap confidence intervals (CI) were obtained for hypothesized indirect effects using 1,000 bootstrap samples.

\section{Considerate voice}

Both the full mediation $\left(\mathrm{M}_{1}\right)$ and partial mediation $\left(\mathrm{M}_{2}\right)$ for considerate voice provided good fit to the current data. For $\mathrm{M}_{1}, \chi^{2}(604, \mathrm{~N}=255)=1104.60, p<.01, \mathrm{CFI}=$ $.93, \mathrm{RMSEA}=.06, \mathrm{SRMR}=.05 ;$ for $\mathrm{M}_{2}, \chi^{2}(600, \mathrm{~N}=255)=1104.12, p<.01, \mathrm{CFI}=.93$, $\mathrm{RMSEA}=.06, \mathrm{SRMR}=.05$. Comparison of the two model indicated that they were not significantly different from each other, $\Delta \chi^{2}(4, \mathrm{~N}=255)=.48, p>.10$, and none of the direct paths in $\mathrm{M}_{2}$ was significant. In addition, although the alternative model $\left(\mathrm{M}_{3}\right)$ had an acceptable fit to the data, $\chi^{2}(607, \mathrm{~N}=255)=1157.84, p<.01, \mathrm{CFI}=.92, \mathrm{RMSEA}=.06$, SRMR $=.11$, the results suggested that $\mathrm{M}_{3}$ fitted the data significantly worse than both $\mathrm{M}_{1}, \Delta \chi^{2}(3, \mathrm{~N}=255)=53.25, p<.01$, and $\mathrm{M}_{2}, \Delta \chi^{2}(7, \mathrm{~N}=255)=53.73, p<.01$. Therefore, I decided to test the hypotheses based on the full mediation model $\left(\mathrm{M}_{1}\right)$.

The standardized regression coefficients are presented in Figure 4. The results in step 1 indicated that the attitude toward considerate voice was significantly related to gender $(\beta=.16, p=.01)$ and normative commitment $(\beta=.25, p<.01)$. With the addition of affective commitment in the model in step 2, gender remained significantly related to attitude toward considerate voice $(\beta=.14, p<.05)$. However, the path between attitude 
toward considerate voice and normative commitment changed its sign (i.e., from positive to negative) and became only marginally significant $(\beta=-2.44, p=.07)$. Finally, affective commitment was significantly related to attitude toward considerate voice $(\beta=$ $3.11, p<.05)$. In step 3, when behavioral expectancies were entered into the model, only gender continued to be significantly related to attitude toward considerate voice $(\beta=.13$, $p<.05)$. In addition, I also found that considerate voice was marginally related to attitude toward considerate voice $(\beta=.13, p=.07)$, which was marginally related to the selfidentity expectancies of considerate voice $(\beta=.25, p=.07)$. The results of step 4 and 5 testing the indirect effects suggested that none of the antecedents (i.e., affective commitment and the three behavioral expectancies) had significant indirect effect on considerate voice. In summary, none of the hypotheses involve considerate voice was supported. The results of these analyses are presented in Table 41 and 44.

\section{Production Deviance}

Both the full mediation $\left(\mathrm{M}_{1}\right)$ and partial mediation $\left(\mathrm{M}_{2}\right)$ for production deviance provided good fit to the current data. For $\mathrm{M}_{1}, \chi^{2}(439, \mathrm{~N}=255)=768.26, p<.01, \mathrm{CFI}=$ $.95, \mathrm{RMSEA}=.05, \mathrm{SRMR}=.05 ;$ for $\mathrm{M}_{2}, \chi^{2}(435, \mathrm{~N}=255)=765.47, p<.01, \mathrm{CFI}=.95$, $\mathrm{RMSEA}=.06, \mathrm{SRMR}=.05$. Comparison of the two model indicated that they were not significantly different from each other, $\Delta \chi^{2}(4, \mathrm{~N}=255)=2.79, p>.10$, and none of the direct paths in $\mathrm{M}_{2}$ was significant. In addition, although the alternative model $\left(\mathrm{M}_{3}\right)$ also had good fit to the data, $\chi^{2}(442, \mathrm{~N}=255)=780.52, p<.01, \mathrm{CFI}=.95, \mathrm{RMSEA}=.06$, SRMR $=.07$, the results suggested that $\mathrm{M}_{3}$ fitted the data significantly worse than both 
$\mathrm{M}_{1}, \Delta \chi^{2}(3, \mathrm{~N}=255)=12.27, p<.01$, and $\mathrm{M}_{2}, \Delta \chi^{2}(7, \mathrm{~N}=255)=15.06, p<.01$.

Therefore, I decided to test the hypotheses based on the full mediation model $\left(\mathrm{M}_{1}\right)$.

The standardized regression coefficients are presented in Figure 5. The results in step 1 indicated that production deviance was significantly related to gender $(\beta=.15, p<$ $.05)$ and attitude toward production deviance $(\beta=.31, p<.01)$. In addition, normative commitment had a negative marginal significant relationship with attitude toward production deviance $(\beta=-.16, p<.06)$. Although production deviance's relationships with gender and attitude toward production deviance remain significant $(\beta=.15, p<.05$ and $\beta=.31, p<.01$, respectively) in step 2 with the addition of affective commitment, attitude toward production deviance was found to be not significantly related to both normative and affective commitment. With the addition of the three classes of behavioral expectancies in step 3, production deviance again was found to be significantly related to gender $(\beta=.15, p<.05)$ and attitude toward production deviance $(\beta=.31, p<.01)$, In addition, attitude toward production deviance was negatively significantly related to selfidentity expectancies $(\beta=-.21, p<.01)$. Step 4's results suggested that the indirect effect of self-identity expectancies of production deviance on production deviance was significant $(\beta=-.06, p<.05)$. However, bootstrap results in step 5 indicated that the $95 \%$ confidence interval of the indirect effect between self-identity expectancies of production deviance and production deviance contained zero, which suggested that the indirect effect was not significant different from zero at $p<.05$. In summary, hypothesis $1 \mathrm{~b}, 2 \mathrm{~b}$ and $3 \mathrm{~b}$ are not supported while hypothesis $4 \mathrm{~b}$ is only partially supported. The results also 
provided limited support for hypothesis $5 \mathrm{~d}$ with regard to production deviance. The results of these analyses are presented in Table 42 and 44.

\section{Behavioral Engagement}

Both the full mediation $\left(\mathrm{M}_{1}\right)$ and partial mediation $\left(\mathrm{M}_{2}\right)$ for behavioral engagement provided acceptable fit to the current data. For $\mathrm{M}_{1}, \chi^{2}(439, \mathrm{~N}=255)=$ $862.31, p<.01, \mathrm{CFI}=.91, \mathrm{RMSEA}=.06, \mathrm{SRMR}=.06 ;$ for $\mathrm{M}_{2}, \chi^{2}(435, \mathrm{~N}=255)=$ $857.14, p<.01, \mathrm{CFI}=.91, \mathrm{RMSEA}=.06, \mathrm{SRMR}=.06$. Comparison of the two model indicated that they were not significantly different from each other, $\Delta \chi^{2}(4, N=255)=$ $5.18, p>.10$, and none of the direct paths in $\mathrm{M}_{2}$ was significant. In addition, although the alternative model $\left(\mathrm{M}_{3}\right)$ also has an acceptable fit to the data, $\chi^{2}(442, \mathrm{~N}=255)=904.67, p$ $<.01, \mathrm{CFI}=.90, \mathrm{RMSEA}=.06, \mathrm{SRMR}=.10$, the results suggested that $\mathrm{M}_{3}$ fitted the data significantly worse than both $\mathrm{M}_{1}, \Delta \chi^{2}(3, \mathrm{~N}=255)=42.36, p<.01$, and $\mathrm{M}_{2}, \Delta \chi^{2}(7$, $\mathrm{N}=255)=47.54, p<.01$. Therefore, I decided to test the hypotheses based on the full mediation model $\left(\mathrm{M}_{1}\right)$.

The standardized regression coefficients are presented in Figure 6. The results in step 1 suggested that behavioral engagement was positively related to attitude toward behavioral engagement $(\beta=.25, p<.01)$, which was found to be positively related to gender $(\beta=.17, p<.01)$ and normative commitment $(\beta=.22, p<.05)$. With the addition of affective commitment in step 2, the result indicated that behavioral engagement remained significantly related to attitude toward behavioral engagement $(\beta=.25, p<$ $.01)$, which was significantly related to gender $(\beta=.13, p<.05)$ and affective commitment $(\beta=2.54, p<.05)$. In addition, the relationship between normative 
commitment and attitude toward behavioral engagement changed its sign (i.e., from positive to negative) and became non-significant. With the three classes of behavioral expectancies added in the model in step 3, behavioral engagement was again positively related attitude toward behavioral engagement $(\beta=.24, p<.01)$, which was positively related to gender $(\beta=.13, p<.05)$ and utilitarian expectancies of behavioral engagement $(\beta=.21, p<.05)$. However, the results in step 4 suggested that the indirect effect from utilitarian expectancies of behavioral engagement to attitude toward behavioral engagement was only marginally significant $(\beta=.05, p=.06)$. Bootstrap results in step 5 confirmed the results in step 4 with the $95 \%$ confidence interval of the indirect effect between utilitarian expectancies of behavioral engagement and behavioral engagement contained zero, which suggested that the indirect effect was not significant different from zero at $p<.05$. In summary, none of the hypotheses involved behavioral engagement is supported. The results of these analyses are presented in Table 43 and 44. 


\section{Chapter 7: Discussion}

The current study investigated the predictive validity of affective commitment, the three classes of behavioral expectancies (i.e., utilitarian, normative and self-identity expectancies), attitude toward behavior (measured by bad-good and harmful-beneficial scale) in predicting several organizational behaviors. Although the results did not support the hypotheses of the current study, the current findings lend support to the argument that the TCM is not qualified as a general model of organizational commitment because of its empirical and theoretical inconsistencies. More specifically, I found affective commitment predicted attitudes toward the behaviors under investigation, while continuance and normative commitment did not. I also found limited evidence for the benefit of adding several classes of behavioral expectancies as predictors of organizational behaviors. The current results suggested that adding behavioral expectancies can better explain attitude toward behaviors but not the behaviors. Finally, I developed and validated the first behavioral engagement scale following the conceptualization of Macey and Schneider (2008).

In the next sections, I first review the results of the scale validation process. Second, I discuss the empirical issues with the TCM which have been reported in the current study. Third, I discuss the findings with regard to the hypotheses. Particularly, I focus on providing alternative explanations for the fact that none of the hypotheses was supported in the current study. Fourth, I provide theoretical and practical implications of the current study. I conclude the chapter with the discussion of the study's limitations and suggestions for future research. 
Scale validation

Following the conceptual framework for scale development recommended by Hinkin (1998), I used several different quantitative analyses such as (a) inter-item correlation analysis; (b) exploratory factor analysis (EFA); and (c) confirmatory factor analysis (CFA) to provide validity evidence for the measures in the current study. In addition, item content analysis and theory-based judgments were also utilized to make decision on whether an item should be kept or removed from the scale. I started with investigating each of the individual scales by looking at their inter-item correlations to identify potential items that do not have strong correlations with other items or correlation patterns suggesting that items are clustered into groups with different meaning. I then used EFA to further explore the factor structure of the measures using maximum likelihood estimator and geomin rotation. Based on the results of the inter-item correlation analysis and the EFA, I decided on which items should be removed from the scales. Bad items usually have low item correlations with other items and low factor loadings (e.g., item 5 and 11 in the scales involved considerate voice). In several scales where items were clustered into groups of similar size (e.g., attitude toward considerate voice), theoretical judgments were made on which group of items is to be removed from the scale.

Bad items or items that do not fit with the content domain of the measures were removed one by one from the scale and EFAs were conducted after each deletion to investigate how the factor structure had changed as the result of the deletion. It is important to note that if a bad item is removed from one scale, it will be removed from all 
other scales relating to the same behavior. For example, in the case of production deviance, although the results indicated that item 5 was a good item in most of the scales involved production deviance (i.e., behavioral expectancies of and attitude toward production deviance), it was still deleted because it was identified as a bad item in the production deviance scale. Next, I conducted CFAs to examine whether the updated scale had a good fit to the data and whether it fitted the data significantly better than the original or other alternative scales. Based on the results of each individual scales, I combined all scales that involved each individual behavior together and use another EFA to investigate whether they were empirically distinguishable from each other. Factor structure and items loadings were examined to ensure items were loaded on their theoretically designated factors. A CFA with modification indices was also conducted to see if the factor structure fitted the data well or not. Modification indices results were examined to identify items that generate large residual covariance with other items. These items, as in the case of considerate voice, were further removed from all the scales involved the same behavior.

The painstaking procedure which I followed is important to ensure the reliability and validity of the measures used in the current study as bad measures are serious threats to our understanding of organizational phenomena (Hinkin, 1995). As a result, for considerate voice, each of the final scales consists of 5 items (i.e., item 1-4 and 8); for production deviance, each of the final scales consists of 4 items (i.e., item 1-4); and for behavioral engagement, each of the final of the final scales consists of 4 items (i.e., items 5-8). As explicated, the final scales that involved each behavior were shown to be clearly 
distinguishable from one another. CFA results suggested the five-factor model (i.e. three behavioral expectancies, attitude toward behavior and the behavior itself) for each behavior had a good fit to the data. Finally, Cronbach's alphas for all measures were above the generally accepted .70 cutoff criteria for acceptable internal consistency, which provided support for the internal reliability of these measures.

There are several conclusions that can be derived from the scale validation process. First, the scale validation results suggest that employees and supervisors do pay attention to the subtle differences across items and scales. For example, employees singled out 2 items (i.e., item 5 and 11) in the scales involved considerate voice, which are different from the rest of the items in that they refer to using compromise in dealing with organization problems. Similarly, the employees indicated that there were possibly two groups of items (i.e., item 1-3 and item 4-8) in the scales involved behavioral engagement. Indeed, as discussed, item 1-3 and item 4-8 refer to very different types of actions: doing something more and doing something different. In addition, for this particular behavior, what fascinating is that while employees recognized the difference between these two groups of items in term of utilitarian and normative expectancies, their ratings suggested that they saw the two groups of items similarly in term of self-identity expectancies. This makes total sense as no matter what you do, something more or something different, you will feel as a good member of the organization. The results also indicated that the approach I took to modify the items to capture the three behavioral expectancies did work as expected. Three behavioral expectancies, although highly correlated, are empirically distinguishable in the current study. 
Second, the results also suggested that there are both consistencies and differences between supervisors' and subordinates' ratings. For example, supervisors agreed with their subordinates that compromising is not a good solution to the problems (item 5 and 11 in the scales involved considerate voice) and that doing something more is different from doing something different (item 1-3 and item 4-8 in the scales involved behavioral engagement). On the other hand, they disagreed with their subordinates regarding whether one should work individually or in a team to deal with organization problems. It appeared that supervisors thought it is important to work in a team to deal with problematic events as they singled out item 9 and 10 from the considerate voice scale. It is consistent with the role of a supervisor who is in charge of the performance of a work unit. In their job, team performance is more important than individual performance. The low inter-item correlations between item 5 and the rest of the items in the production deviance scale suggested that supervisor had difficulty in rating their employees using this item because it is more private and very difficult to observe. An implication for the differences in ratings between employees and supervisors for counterproductive work behaviors (CWB) and organizational citizenship behaviors (OCB)-like behaviors is that the supervisors' ratings can be a source of error as many of these behaviors are private and thus unobservable to the supervisors (Dalal, 2005). The results, however, suggested that supervisors in the current sample did not make the judgment about production deviance based on a general impression of employees (cf. Sackett, Berry, Wiemann, \& Laczo, 2006) because otherwise it could have caused item 5's correlations to be of similar size as those of other items. 
Third, I used two different scales to evaluate attitude toward behavior, bad-good and harmful-beneficial. Between the two scales, bad-good scale is considered to have the ability to capture both components of attitude (i.e., instrumental and affective) while harmful-beneficial scale tends to capture the instrumental component (Ajzen, 2002). My results suggest that respondents in the current sample may respond differently to the two components of attitude toward behavior, depending on the type of behavior in question. More specifically, for considerate voice, EFA results suggested a two-factor model indicating that the two scales captured two different aspects of attitudes. On the other hand, for production deviance and behavioral engagement, EFA results suggested a onefactor model indicating that the two scales were highly related to one another and capture a similar attitude. The nature of these behaviors appears to be the reason for the current findings. Considerate voice involves behaviors relating to problem solving at work that takes both interests of individual and the organization into consideration. Because interests from both sides are often in conflict with one another, employee may think that good behaviors do not always maximize their benefits. For example, a good behavior responding to a budget cut due to economic downturn would be "Trying to come to an understanding with parties related to the problem". However, an understanding of the situation that the organization is facing would mean to accept certain level of pay cut or reduced working hour, which is not the best solution if one considers his/her benefits. On the other hand, for production deviance and behavioral engagement, which do not have such conflicting interests, engaging in a good behavior usually bring good benefits, and vice versa, engaging in a bad behavior usually bring negative outcomes. I decided to use 
only harmful-beneficial scale to capture attitude toward behavior in the current study because it has the potential to provide clearer interpretation of the relationship between attitude and other measures. Using bad-good scale may contaminate our understanding of the nomological network among measures because research has found that the two components have differential relationships with organizational and individual outcomes (Glasman \& Albarracin, 2006; Kraft et al., 2005; Lawton et al., 2009).

Fourth, an important contribution of the current study is the development of a new behavioral engagement scale based on Macey and Schneider's (2008) conceptualization. More specifically, I based on propositions 7-10 in Macey and Schneider (2008) to develop 8 items for the original behavioral engagement scale. As discussed earlier, the results suggested a two-factor model with item 1-3 loaded on one factor and item 4-8 loaded on the other factor. Item content analysis suggested that the factor on which item 1-3 loaded was referring to doing something more (e.g., working extra hours) for the organization and the factor on which item 4-8 loaded was referring to doing something different (e.g., proactive, innovative and adaptive behaviors) for the organization. The study's results fit with Macey and Schneider's (2008, p. 15) contention that "it is limiting to define engagement solely in terms of "extra effort" that is, just doing more of what is usual". Although I only used items 5-8 in the current study, future research could use both components (i.e., doing something more and doing something different) to further validate and investigate the construct of behavioral engagement, especially on the discriminant validity between behavioral engagement and similar constructs such as OCB. 


\section{Summary of scale validation}

I have followed comprehensive scale validation procedures to ensure the reliability and validity of the measure for the current study. The results of the validation procedures suggest that attention should be paid in order to choose an appropriate source of ratings for counterproductive work behavior and behavioral engagement. As will be discussed in more details in the next sections, some of the findings in the current study might be attributable to the discrepancy between sources of ratings. In addition, care should be taken to choose appropriate adjectives pairs (e.g., harmful - beneficial) to measure attitude toward behaviors. I would recommend using adjective pairs to capture both components of the attitudes whenever it is possible (Ajzen, 2002). Finally, more measurement development is needed to further develop and improve the behavioral engagement scale, especially generating more items to further capture behavioral engagement domain. I would suggest future research generate more items based on proactive, innovative and adaptive behaviors. It is possible that these behaviors can be further developed into separate dimensions under the latent construct of behavioral engagement.

\section{Measurement Model Testing}

Following Anderson and Gerbing's (1988) two-stage procedure for structural equation modeling, the overall measurement model $\left(\mathrm{M}_{0}\right)$ that included all 8 intended latent constructs (i.e., three behavioral expectancies, attitude toward behavior, the behavior, and three components of organizational commitment) for each behavior was estimated. The results, however, indicated that none of these models had a sufficient fit to 
the current data. Because all the models without organizational commitment had shown good model fit, I focused my attention on investigating the factor structure of organizational commitment scale. Initial examination indicated that the three-factor model of organizational commitment did not fit the data and EFA results indicated several issues with the three components of organizational commitment. More specifically, the results suggested that all the negatively worded items (i.e., item 3, 4, and 6 from affective commitment scale and item 1 from normative commitment scale) did not function as expected. In addition, I also encountered the dimensionality issue with continuance commitment scale in which items reflecting lack of alternatives and items reflecting high sacrifices were loaded on two different factors. Finally, several items from normative commitment scale (i.e., item 4, 5, and 6) were found to be loaded on the same factor with several items from affective commitment scale (i.e., item 1, 2, and 5). These issues appeared to be the reasons for the lack of fit of the overall measurement models. Based on the results, I decided to remove all negative worded items from the scales and used only item 1-3 (lack of alternatives) from continuance commitment scale for testing the hypotheses of the study. Updated measurement models with the new organizational commitment scale were estimated again and the results indicated that all models had a good fit to the data.

While disappointing, the issues with the organizational commitment scale in the current study are consistent with the empirical evidence in the literature, especially when the scale is generalized into a different culture. With regard to the relationship between affective and normative commitment, several earlier attempts to validate organizational 
commitment in South Korea (e.g., Ko et al., 1997; Lee et al., 2001; Lee \& Yang, 2005) have reported the same problem with the lack of discriminant validity between affective and normative commitment. This issue is a special case of the concept redundancy problem that has been frequently reported in the commitment literature (Cooper-Hakim \& Viswesvaran, 2005). In addition, similar to McGee and Ford (1987), the results in the current study indicated that continuance commitment has two sub-dimensions, which might lead to the low convergent validity problem. Finally, I found that all the negatively worded items were problematic in the current study. These items can distort the perception of respondents and affect their ratings accordingly. For instant, item 3 in the affective commitment scale - "I do not feel like "part of the family" at my organization" - may make respondents perceive that agreeing to this question means that they must have a bad relationship with the organization, where in fact they need not. As Socrates have pointed out, "what is not beautiful need not be ugly and what is not good need not be bad", therefore, not feeling like "part of the family" does not necessarily mean having a bad relationship with the organization and vice versa. The distortion effect of negatively worded items might have even been exacerbated by the collectivistic culture in China where the loyalty with the organization is highly valued. In summary, the results of measurement model testing suggest serious issues with the organizational commitment scale of the TCM which might not be fixed by simply modifying the measurement of the scales (Solinger et al., 2008). 
Affective commitment and the three organizational behaviors

I predicted in Hypothesis 1 that employees' affective commitment will be positively related to considerate voice and behavioral engagement and negatively related to production deviance. The results, however, indicated that Hypothesis 1 was not supported. Examination of past research with regard to the relationship between general attitudes and specific behaviors suggested that the low predictive validity of affective commitment is not something new in the attitudinal literature. In fact, both qualitative (e.g., Brayfield \& Crockett, 1955) and quantitative (e.g., Iaffaldano \& Muchinsky, 1985; Mathieu \& Zajac, 1990) reviews of the relationship between attitudes and behaviors have reported weak relationships between job attitudes (e.g., job satisfaction and organizational commitment) and various performance criteria. Ajzen and Fishbein (2005, p. 178) termed the lack of predictive validity of general attitudes for specific behaviors evaluative inconsistency because "it involves the lack of correspondence in evaluation expressed in verbal attitudes and in actual behavior". In the current study, except for production deviance whose non-significant relationship with affective commitment could have caused by the low base rate of production deviance $(M=1.10, S D=.26)$, evaluative inconsistency between affective commitment and the three organizational behaviors in the current study may be attributable to (a) the lack of compatibility between affective commitment and the specific behaviors, (b) the existence of moderators such as individual differences and contextual characteristics such as national culture, and (c) discrepancy between self-ratings and supervisor ratings. 
First of all, the lack of compatibility between attitude and behaviors in term of target, action, context and time has been considered as the prime reason for evaluative inconsistency in the attitude-behavior relationship (Early \& Chaiken, 1993, Ajzen \& Fishbein, 2005). In fact, most of the earlier studies reviewed by Wicker (1969) encountered this inconsistency issue when trying to link attitudes toward broad social issues such as racial discrimination or labor union and specific behaviors such as taking pictures with an African American of the opposite sex (e.g., DeFleur \& Westie, 1958) or attending labor union meeting (e.g., Dean, 1958). The current study appears to face the same issue in investigating the relationship between affective commitment and the three organizational behaviors. On one hand, affective commitment is a general attitude of an employee vis-à-vis an organization (i.e., target) without any particular action, context, and time (Solinger et al., 2008). On the other hand, the organizational behaviors examined in the current study are more specific not only in terms of their actions but also the contexts in which these behaviors may exhibit. More specifically, the context for considerate voice is situation where employees face with problematic events at work such as interpersonal conflict or unfair pay cut. Similarly, for production deviance, the context is usually a negative event. Research has found evidence suggesting that different types of CWBs tend to have different types of antecedents, or in other words, happen in specific contexts (e.g., Spector et al., 2006). For example, Spector et al. found that interpersonal conflict and job stressors were the key antecedents that activate production deviance in which employee purposefully fail to perform job tasks effectively. Finally, the context for behavioral engagement are situations where employees facing with 
organizational challenges and opportunities. As can be seen in the questionnaire for the supervisors under the Appendix, the contexts of the three organizational behaviors have been defined and communicated clearly to the supervisors prior to rating, and thus the behaviors' ratings should closely reflect the specificity of the behaviors' contexts. In short, the contexts in which the three organizational behaviors may exhibit are highly specific making it incompatible with affective commitment in terms of action and context. For that reason, according to the principle of compatibility (Ajzen, 1988; Ajzen \& Fishbein, 1977), it is conceivable that affective commitment is not predictive of the three behaviors in the current study.

The current results with regard to the relationship between affective commitment and the three organizational behaviors are consistent with the current literature on the role of job attitudes in predicting behavioral outcomes. For example, in comparing several diversified models where specific behaviors (e.g., focal and contextual performance) are determined separately by job attitudes and unified models where the same behaviors were aggregated to be determined by job attitudes, Harrison et al. (2006) found that a general set of behaviors at work - not specific behaviors - is the best criterion for the overall job attitude. Based on the results, Harrison et al suggests that "when attempting to understand patterns of work behavior from attitudes such as job satisfaction and organizational commitment, researchers should conceptualize the criterion at a high level of abstraction" (p. 316).

The second factor that may have contributed to the lack of predictive validity of organizational commitment is the possibility of having moderators in the relationship 
between affective commitment and the behaviors of interest in the current study. Moderating variables may include the individual differences, the background factors of the situation in which the behavior is performed, or the nature of the attitude (Ajzen \& Fishbein, 2005). Among these possible moderators, national culture appears to be a viable explanation for the lack of predictive validity of affective commitment because a Chinese sample was utilized for the current study. Research by Fazio (e.g., 1986, 1990) has proposed that social norms (e.g. national culture) may moderate the relationship between attitudes toward target and the behaviors through its impact on the definition of the event - "the perceptions that involve both the attitude object and the situation in which the object is encountered" (Fazio, 1986, p. 208). Cross-cultural organizational behavior research has documented abundant evidence with regard to the effects of national culture on the nature of the relationship between individuals and organizations. In particular, several cultural dimensions under Hofstede's (1980) cultural framework such as individualism/collectivism and power distance have been shown to moderate how people perceive and react to various organizational processes and events (Gelfand et al., 2007). According to Hofstede (2011), Chinese rank lower in individualism dimension and higher in power distance dimension (scored 20 and 80 respectively) than many Western countries such as Australia (scored 90 and 36 respectively), United States (scored 91 and 40 respectively), or United Kingdom (scored 89 and 36 respectively). For that reason, it is plausible that the national culture may have moderated the relationship between affective commitment and the organizational behaviors in the current study in several different ways. 
The relationship between affective commitment and considerate voice could be influenced by both power distance and individualism/collectivism dimension. First, because of high power distance culture, Chinese employees are less likely to voice their concerns to their superiors when facing with organizational problems such as inconvenient working schedule or unfair pay cut. Rather, employees tend to be more patient to wait for the problem to resolve itself or trust the organization and its agents that they will take care of the problem. Such tendency fits better with a different category of response to organizational problems which Farrell (1983) and others named loyalty ${ }^{2}$. In addition, research has found that being able to voice concerns are less important for individuals in high as compared with low power distance cultures (Brockner, Ackerman, Greenberg, Gelfand, Francesco, et al., 2001). In a similar fashion, individualism/collectivism has been linked with differences in how individuals from different cultures deal with disputes and conflicts at work. More specifically, research indicated that individuals within high collectivistic cultures tend to rely more on their leaders to resolve conflict while individuals within high individualistic cultures prefer integrating interest approach where the interest of both parties are considered in order to reach an agreement (Tinsley, 1998, 2001). Similarly, Holt and Devore (2005) in their recent meta-analysis found that people in individualistic cultures tend to choose forcing as a conflict resolution style more than those in collectivistic cultures. On the other hand, individuals in collectivistic cultures prefer withdrawing, compromising and problem-

\footnotetext{
${ }^{2}$ It is important to note that although the mean ratings of considerate voice is high $(M=5.92 ; S D=.91)$, it does not necessarily mean that considerate voice is the most favorable approach to deal with organizational problems because no information on the frequency of such behavior is collected and compared with other alternatives such as loyalty.
} 
solving more than those in individualistic cultures. In summary, because of the national culture, the respondents in the current study may prefer a different course of action such as loyalty to voicing directly to their superiors and the organization to deal with the problems, thus making affective commitment less predictive of considerate voice in the current study.

The relationship between affective commitment and production deviance could be influenced by individualism/collectivism and/or power distance dimension. Research has found that managers in China placed less emphasis on CWB in rating employees' performance than did North America managers (Rotundo \& Xie, 2008). Therefore, it is reasonable to expect that Chinese manager will put less emphasis on establishing policies and procedures to prevent CWBs from happening and punish employees who commit CWBs more leniently. Such practices will encourage CWBs because employees who perceived they are unlikely to be caught, or if caught, are unlikely to be punished severely are more likely to engage in CWBs (Fine, Horowitz, Weigler, \& Basis, 2010). In high collectivistic cultures where individuals are expected to conform to group's norms, individuals with high affective commitment may still engage in production deviance. In other words, the negative relationship between affective commitment and production deviance could be weakened and became not significant. In an opposite direction, the non-significant relationship between affective commitment and production deviance could be explained by the high power distance culture in China. In high power distance cultures, followers are more likely to accept and expect unequal power and unequal treatment between them and their leaders (Hofstede, 2011). Therefore, individuals with 
low affective commitment as a result of injustice treatment are less likely to engage in retaliation behaviors, making affective commitment less predictive of production deviance. As suggested by production deviance ratings $(M=1.10 ; S D=.26)$, most of the respondents in the current study have never engaged in production deviance.

The relationship between affective commitment and behavioral engagement could be influenced by individualism/collectivism dimension. As discussed, behavioral engagement is often linked with the notion of "going beyond the usual or typical" (Macey \& Schneider, 2008). In this aspect, behavioral engagement encompasses several related constructs such as extra role behaviors, organizational citizenship behaviors (OCBs), or proactive behaviors. Research (e.g., Lam, Hui, \& Law, 1999) with regard to the structure and dimension of extra role or citizenship behaviors has found that individual in high collectivistic cultures (e.g., Hong Kong and Japan) tend to considered some of the dimension of OCBs (e.g., courtesy and sportsmanship) as components of task performance. For this reason, some of the actions under behavioral engagement could have been considered as part of the formal job description in the current study. In addition, affective commitment has been shown to be more strongly related to OCBs than to task performance because it reflects employees' emotional attachment to the organization (Meyer et al., 2002). Taken together, it is reasonable for affective commitment to correlate less strongly or not at all with behavioral engagement in collectivistic cultures where behavioral engagement is considered as a part of the task performance. Additionally, Meyer et al. (2002) also found that normative commitment predicts OCBs more strongly than affective commitment. Because I controlled for 
normative commitment, the relationship between affective commitment and behavioral engagement could become non-significant.

In addition, the response tendencies to the measures (i.e., participants' tendencies in responding to the survey items) in the current study might also be influenced by individualism/collectivism dimension. Research by Ting-Toomey and colleagues (e.g., Cocroft \& Ting-Toomey, 1994; Ting-Toomey, Gao, Trubisky, Yang, Kim, Lin et al., 1991) indicated that people in collectivistic cultures tend to use more other-oriented facesaving and face-honoring strategies while people in individualistic cultures tend to use more self-oriented face-saving strategies. In other words, collectivists tend to be more concerned about other-face rather than self-face. In addition, in high collectivistic cultures where group harmony is of utmost important, supervisors are more inclined to make others (i.e. their subordinates) look good in order to maintain the group harmony. Thus, they are more likely to rate their subordinates higher on positive behaviors such as considerate voice and behavioral engagement and lower on negative behaviors such as production deviance. As a result, the inflated (or suppressed) ratings could have influenced the relationships between affective commitment and the three classes of behavioral expectancies and the three organizational behaviors investigated in the study.

Finally, the non-significant relationship between affective commitment and the three organizational behaviors could be the result of the discrepancy between supervisors' and self-ratings. Conway and Huffcutt (1997) found the mean correlation between supervisor ratings and self-ratings was low at .22. Therefore, the relationship 
between affective commitment and the organizational behaviors could have been higher if I had used self-ratings of the behaviors.

\section{Behavioral expectancies and the three organizational behaviors}

In Hypotheses 2, 3 and 4, three classes of behavioral expectancies (i.e., utilitarian, normative, and self-identity expectancies) were hypothesized to be related to the three organizational behaviors (i.e., considerate voice, production deviance, and behavioral engagement). However, except for the significant indirect effect between anticipated selfidentity of production deviance and production deviance behavior, path-analysis results indicated that none of these hypotheses were supported. Moreover, bootstrap results indicated that all the confidence intervals of these estimates contain zero. Therefore, it can be concluded that Hypotheses 2, 3, and 4 were not supported. In the following section, I will further look at each of the hypotheses and provide alternative explanations for and interpretation of the current findings with regard to the relationships between these classes of behavioral expectancies and the behaviors.

Hypothesis 2 involves the relationship between utilitarian expectancies and the behaviors. More exactly, drawing from expectancy-value theory (EVT; see Feather, 1982), I hypothesized that the higher rewards an individual anticipates gaining in engaging a certain behavior, the more likely he/she will engage in the behavior. This hypothesis was not supported for any of the three behaviors in the current study. Examination of the behaviors and their context, however, suggests some explanations for the current findings. First of all, as explained in the previous discussion of the potential impacts of national culture, the respondents in the current study may not think the 
behaviors investigated in the current study are the best solutions to address their corresponding problems. For example, they may prefer loyalty to considerate voice, nonCWBs to production deviance, or simply working extra hours to taking risk with new responsibility. As a result, utilitarian expectancies might not predict well the behaviors of interest in the current study.

Alternatively, respondents in the current study may have felt that they lacked the resources (e.g., knowledge, skills, and ability) and control to successfully perform these behaviors. More specifically, respondents may think the possibility of influencing their leaders and the organization by voicing their concern is small and the gained benefits would not be as great compared to other options such as loyalty in which they trust and let the organization to solve the problems for them. In a similar fashion, respondents might think that they do not have necessary control over production deviance and are afraid of being caught. Finally, for behavioral engagement - which captures the proactive, adaptive and innovative behaviors - respondents might have thought that they lacked of the required experience, knowledge, skills and ability to engage in the behavior successfully. The inability of the Theory of Reasoned Actions (TRA) to predict nonvolitional behaviors that requires resources and control have been criticized by attitude researchers (e.g., Liska, 1984). Sensitive to such criticism, Ajzen $(1985,1991)$ has proposed the Theory of Planned Behaviors (TPB) to include perceived behavioral control as an additional predictor of the behavioral intention and actual behavior. Alternatively, this explanation also fits with expectancy theory (Vroom, 1964) which posits that employees will not be motivated to engage in certain behaviors if they do not think they 
have the ability to do so, even when successful performance could lead to certain outcomes of great valence to them.

There are also other explanations that are more relevant to specific behaviors. For production deviance, a possible reason is that the employees were happy with their leaders and the organizations which reduced the need to engage in aggressive and negative responses to the organization. As indicated by the mean ratings of production deviance $(M=1.10, S D=.26)$, production deviance almost never happen among the sample of the current study. Another possible reason for the non-significant relationship between production deviance and its utilitarian expectancies is the lack of variability in supervisors' ratings. If there is little or no variance in the behavior, strong relationship cannot be expected (Ajzen \& Fishbein, 2005). Finally, the lack of predictive validity of utilitarian expectancies for behavioral engagement could be explained by the possibility that behavioral engagement is considered as a part of the task performance domain in the current organization (see Lam et al., 1999). Being part of job responsibility means little or no extra rewards for behavior engagement and it could explain the non-significant relationship between behavioral engagement and its utilitarian expectancies found in the current study.

Hypothesis 3 involves the relationship between normative expectancies and the behaviors. The central thesis of this hypothesis is the recognition of the importance of social processes such as peer pressures and groups norms in shaping one's attitudes and behaviors. While the hypothesis was not supported, there are several factors may be responsible for these findings. First, as discussed in the previous section, three 
organizational behaviors in the current study may not be evaluated as favorably as some other alternative behaviors not included in the current study as a result of the national culture. Therefore, it is possible that social norms exert their influence on a different set of actions rather than the behaviors in the study, resulting in the non-significant relationship between the behaviors and their normative expectancies. Alternatively, as the original model of Theory of Reason Actions (Fishbein \& Ajzen, 1980) suggested, normative expectancies of behaviors could impact the behavior through intentions, which is not tested in the current study. It is quite possible that intention is not a good predictor of behavior, especially when individuals consider the lack of control in the performance of these behaviors. Ajzen and Fishbein (2005) referred to this issue as literal inconsistency when people say they do something but end up doing something else. Research (e.g., Ajzen, Brown \& Carvajal, 2004; LaPiere, 1934) has reported similar issues with literal inconsistency. Finally, the lack of predictive validity of normative expectancies could come from an attribute that is inherent to the normative expectancies scales in the current study. Investigators have distinguished the two types of normative pressures: injunctive (i.e., perceptions of what others think one should do) and behavioral or descriptive (i.e., perceptions of what others are doing). Ajzen and Fishbein (2005) argue that it is important to include items that tap on both types of norms to have a complete measure of normative expectancies. As described in the measure section, only behavioral measure of normative expectancies is included in the current study. Hypothesis 4 concerns the relationship between anticipated self-identity expectancies and behavior. The hypothesis is based on identity theory (Stryker \& Serpe, 
1982) and role-identity theory (McCall \& Simmons, 1978) which both argue for the importance of maintaining a positive identity within one's social network, especially in the contexts that are important to the individual. The results, however, indicated that Hypothesis 4 was not supported. There are several reasons that may provide explanations to the current findings. First of all, as the composite attitude - behavior model of Eagly \& Chaiken (1993) suggested, self-identity expectancies could exert its influence on behaviors through behavioral intentions. As discussed earlier, intention might not be a good predictor of the behaviors, especially when you do not have necessary resources and control to perform the behaviors successfully. For this reason, self-identity expectancies might not be able to predict the behaviors of interest. Second, examination of the nature of the three behaviors and the results of the current study suggests that the relative importance of the behavioral expectancies (i.e., utilitarian, normative, and self-identity expectancies) in the current study may be a function of the behavior and the population under investigation (Ajzen \& Fishbein, 2005). For behaviors that require extra responsibility and efforts like behavioral engagement, the anticipated rewards from engaging the behavior are likely to be more salient to the respondents, making utilitarian expectancies to be more relevant in predicting this type of behavior. On the other hand, for behaviors relating to moral judgments (i.e., right vs. wrong) such as considerate voice and production deviance, self-identity expectancies would be more relevant because it reflects whether an individual identify his/herself with the action. Empirical evidence in the current study partly supports this argument. More specifically, the result indicated that only utilitarian expectancies predict attitude toward behavioral engagement while 
self-identity expectancies predict attitude toward the other two behaviors (albeit only marginally for considerate voice). For that reason, it is reasonable to find self-identity expectancies did not predict behavioral engagement. With regard to production deviance and considerate voice, although the results indicated that these behaviors were not significantly related to their self-identity expectancies, the findings may be attributable to other reasons (e.g., identification with alternative behaviors, lack of resource and control over the performance, or the low base rate of production deviance) rather than the ability of self-identity expectancies to predict the behaviors.

\section{Mediating role of attitudes toward behaviors}

Hypothesis 5 tested the indirect effects from affective commitment (i.e., attitude toward target) and three classes of behavioral expectancies on the behavior through attitude toward behavior. It was also a test of the principle of compatibility (Ajzen \& Fishbein, 1977) which suggests that attitudes can only impact behaviors when they are measured at a similar level of generality or specificity. The bootstrap results, however, indicated that the indirect effects were not significant, and therefore Hypothesis 5 was not supported. Mathematically, an indirect effect between a predictor (X) and an outcome (Y) through a mediator $(\mathrm{M})$ is the product of the path from $\mathrm{X}$ to $\mathrm{M}$ and the path from $\mathrm{M}$ to $\mathrm{Y}$ (Preacher \& Hayes, 2004). The non-significant indirect effects could be attributable to factors that weaken either or both paths from $\mathrm{X}$ to $\mathrm{M}$ and from $\mathrm{M}$ to $\mathrm{Y}$. The reasons below are identified as potential factors that caused the non-significant indirect effects in the current study. 
First of all, as mentioned in the previous sections, it is possible that the respondents in the current study did not think that considerate voice, production deviance and behavioral engagement are the best answers to their corresponding issues. Instead, they may prefer a difference course of actions such as loyalty, non-CWBs, or simply working extra hours to deal with the demand of the events. As discussed, their preferences may be influenced by the national culture of the sample or the organizational context and social network in which they interact. If the behaviors in the current study are not the usual actions that respondents take to deal with their corresponding problems, the strength of the paths from $\mathrm{X}$ to $\mathrm{M}$ and from $\mathrm{M}$ to $\mathrm{Y}$ will not be large. This would explain why the indirect effects between the predictors (i.e., affective commitment and the three classes of behavioral expectancies) and the behaviors were not significant. It could have been possible to find attitude toward behavior to mediate such relationships if I had chosen are different set of behaviors that are more appropriate for the events from the perspective of the respondents.

Second, because all the behaviors in the current study require some sort of resources, control, cooperation or skills, it is reasonable to expect perceived behavioral control - perception of how easy of difficult it is to perform the behavior - contributes more significant variance to the prediction of the behaviors (Ajzen, 1985, 1991; Ajzen \& Fishbein, 2005). Both the theoretical model and the empirical evidence of the Theory of Planned Behaviors suggested that perceived behavioral control tend to influence behavior directly or through behavioral intentions but not through attitude toward behavior. Therefore, the paths from $\mathrm{X}$ to $\mathrm{M}$ and from $\mathrm{M}$ to $\mathrm{Y}$ might be trivial in the current study 
when the perceived behavioral control is a relatively more important predictor of the behaviors. Unfortunately, I did not include both intention and perceived behavioral control in the current study.

Third, the behaviors of interest in the current study might have been new to the respondents and they may have never engaged in similar actions before. Therefore, it is reasonable to believe that the respondents may not have direct experience with the behaviors. Moreover, it is also likely that attitudes toward behaviors in the current study were newly formed and reported for the first time. On the other hand, because the attitudes toward behaviors in the current study were collected around the same time with the behaviors, the behavior ratings by supervisors are more likely to reflect their overall evaluation of employees in term of the behaviors in the past. According to the recent meta-analysis by Glasman and Albarracin (2006), the lack of direct experience with the behavior, the newly formed and first time reported attitudes, and the ratings of past behaviors are factors that may lower the accessibility and stability of attitude, which in turn lower the relationship between attitude and behaviors (i.e., the path from M to Y).

Fourth, I use supervisors' ratings in the current study to avoid common method bias. However, the use of supervisors' ratings might also lead to lower relationship between attitude and behaviors (i.e., the path from M to Y) because of the discrepancy between two sources of ratings. Research by Conway and Huffcutt (1997) found the mean correlation between supervisor ratings and self-ratings was low at .22 .

Finally, as indicated in the correlation analysis, I found that affective commitment, normative commitment, and three behavioral expectancies of the three 
behaviors are highly correlated with one another. For example, correlations among behavioral expectancies of considerate voice were around .70 or the same correlations for behavioral engagement were around .50. Therefore, the low correlations between the predictors and mediators may be attributable to a multicollinearity problem.

\section{Summary of Hypothesis Testing}

I have provided several alternative explanations for the findings of the current

study, especially on why the hypotheses were not supported. In general, the study seems to have encountered several issues which may independently or jointly affect its results.

First of all, it appears that the lack of compatibility between attitude and behaviors is the most important factor that led to the evaluative inconsistency issue with the relationship between affective commitment and the three behaviors in the current study. This problem has been noted by Solinger et al. (2008) as a potential limitation of the Eagly and Chaiken's (1993) model. Second, the existence of moderators (e.g. national culture) could influence the relationship between the predictors (i.e., affective commitment and behavioral expectancies) and the behaviors in the current study. More specifically, the uniqueness of Chinese culture could influence the way that respondents in the current study choose to respond the organizational events. They may prefer a different set of behaviors that are more culturally correct than the behaviors in the current study. Third, as all the behaviors examined in the current study need certain level of resource (e.g., knowledge, skills, ability) or cooperation to perform successfully, perceived behavioral control seems to be a relatively more important predictor for these behaviors. As a result, the predictors in the current study would be less likely to significantly predict these behaviors. If it was included in the model, perceived behavioral control could have 
contributed a greater variance to the prediction of the behaviors, compared with those of the current predictors. Fourth, several predictors in the current study (i.e., normative and self-identity expectancies) could influence the behaviors through behavioral intentions, which could reduce the role attitude toward behavior in the relationship between these predictors and the behaviors. Fifth, the lack of direct experience with the behavior, the newly formed and first time reported attitudes, and the ratings of past behaviors may lower the accessibility and stability of attitude, which in turn lower the relationship between attitude and behaviors. Sixth, the relationship between attitudes toward behaviors and the behaviors could be lessened because supervisor ratings was utilized and collected at the same time with the attitudes toward behaviors. Seventh, the multicollinearity issue among study's predictors and the lack of variability of production deviance could also have lowered the relationship between the studies variables.

Although none of the hypotheses was supported, I found the current findings provide limited supports for my arguments with regard to the empirical and conceptual shortcomings of the TCM as well as for the reconceptualization of organizational commitment strictly as the attitude toward the organization (Solinger et al., 2008). First of all, the current findings suggest serious empirical issues with the TCM which may not be resolved through modifying its measurement or interpreting in term of motivational term (Solinger et al., 2008). In the current study, the issues with the TCM include the lack of discriminant validity between affective and normative commitment, the dimensional issue with continuance commitment, and the problems with the negatively worded items in the scales. Second, I found limited evidence to support the argument that affective 
commitment should be considered as a sole indicator of organization commitment. Indeed, affective commitment significantly predicted attitudes toward considerate voice and behavioral engagement in the current study, controlling for continuance and normative commitment. Third, the results also indicated that continuance and normative commitment as attitudes toward the act of leaving did not significantly predict attitude toward considerate voice and behavioral engagement, controlling for affective commitment. Therefore, including continuance and normative commitment under the same attitudinal label with affective commitment is problematic. Finally, the significant relationship between behavioral expectancies and attitude toward behaviors (i.e., utilitarian expectancies for behavioral engagement or self-identity expectancies for production deviance and considerate voice), controlling for affective, continuance, and normative commitment suggests that we can better predict organizational behaviors by adding appropriate behavioral expectancies to the model.

\section{Theoretical Implications}

As discussed in the previous sections, it appeared that the theoretical inadequacy of Eagly and Chaiken's (1993) model was one of the main reasons for the lack of support for the study's hypotheses. In this section, I discuss several important theoretical implications of the current study with a special focus on improving the theoretical model suggested by Eagly and Chaiken (1993). I also suggest several methodological solutions for the measurement issues encountered in capturing several components of the Eagly and Chaiken's model (i.e., attitude toward behavior and normative expectancies). First, the findings of the current study illustrated the empirical and conceptual shortcomings of 
the TCM and provided some empirical evidence (i.e., affective commitment significantly predict attitudes toward behaviors, controlling for continuance and normative commitment) for the reconceptualization of organizational commitment as suggested by Solinger et al. (2008). Under this reconceptualization, affective commitment is the only attitude toward target in the TCM and therefore should be considered as the sole indicator of organizational commitment. Also the results suggested that attitude toward behavior is superior to attitude toward target in predicting specific behavior. Taken together, the current study provides some support for using Eagly and Chaiken's (1993) as a general model to predict organizational behaviors. One of the most important contributions of Eagly and Chaiken's model is its ability to distinguish attitudes toward targets and attitudes toward behaviors, and link the two types of attitudes in a causal chain to predict organizational behaviors (Solinger et al., 2008).

Various recent attempts by the proponents of the TCM (e.g., Meyer \& Herscovitch, 2001; Meyer \& Parfyonova, 2009; Powell \& Meyer, 2004) suggest that work is underway on their parts to radically change the conceptualization and measurement of the TCM. For example Meyer and Parfyonova (2009) suggested that normative commitment has a "dual nature" that manifests itself depends on the strengths of affective and continuance commitment. This conceptualization suggests that one component of organizational commitment (e.g., continuance commitment) may moderate the relationships between the other component (i.e., normative commitment) and its outcomes. The new conceptualization of normative commitment, therefore, is not consistent with the earlier additive model of the TCM where a person's total commitment 
is considered as the 'net sum' of the three psychological states. Indeed, Meyer and his colleagues have strayed away from using the term 'total commitment' to describe one's overall organizational commitment. Rather, "organizational commitment profile" is currently a preferred term to characterize organizational commitment (Meyer \& Herscovitch, 2001; Meyer \& Parfyonova, 2009). However, as argued by Solinger et al. (2008), unless the distinction between affective commitment as an attitude toward target and continuance and normative commitment as attitudes toward behavior is recognized by the TCM, the model is conceptually inappropriate to be qualified as a general model of organizational commitment.

Second, my results provided some support for the addition of behavioral expectancies in the model to better explain the behaviors of interest (Eagly \& Chaiken, 1993; Solinger et al., 2008). However, the results also suggested that the relative importance among utilitarian, normative, and self-identity expectancies may be a function of the behavior and the population under investigation (Ajzen \& Fishbein, 2005). Therefore, it might not be a good idea to include all three behavioral expectancies in the model as it may create the problem of multicollinearity. In the current study, I found that self-identity expectancies was more relevant to behaviors involving to moral judgment while utilitarian expectancies was more relevant to behaviors that require investment of some sorts such as time, skills and efforts. The current findings could be used as a basis to generate more hypotheses with different behaviors in future research.

Third, the results of my dissertation suggest that future research might encounter issues similar to those in the current study (i.e., low or non-significant relationship 
between organizational commitment and specific behaviors), if researchers follow Solinger et al.'s (2008) recommendations on extending the range of behaviors to be explained by organizational commitment. My contention is that it will be unlikely to find moderate or even small significant relationships between organizational commitment and specific organizational behaviors unless the behaviors are aggregated at a certain level that is more compatible with organizational commitment (Harrison et al., 2006). In other words, high correlations between organizational commitment and organizational behaviors should only be expected when we define our behaviors at higher-order levels such as task performance, citizenship performance, and counterproductive work behaviors.

Fourth, as most of the organizational behaviors require certain level of resources, control and cooperation, perceived behavioral control should be added as an antecedent in the Eagly and Chaiken's (1993) model. It is anticipated that the addition of perceived behavioral control will improve the prediction of organizational behaviors considerably. However, as indicated by the Theory of Planned Behaviors (Ajzen, 1985, 1991), it is unlikely that perceived behavioral control will impact the behavior via attitude toward behavior, but rather via behavioral intention or directly on the behavior.

Fifth, it is important to realize that the Theory of Reasoned Action (TRA) and Theory of Planned Behaviors (TPB) were designed as general theories of attitude to apply in a wide range of situations and settings (e.g., social, organizational, educational, medical). According to these theories, the major and proximal predictors of intentions and behaviors only follow from behavioral, normative, and control beliefs. In fact, Ajzen 
and Fishbein (2005) pointed out that these theories do not address the vast number of potentially relevant background factors because "it is difficult to know which should be considered without a theory to guide selection in the behavioral domain of interest" ( $p$. 197). Therefore, in applying these theories to organizational setting (i.e., job attitudes), it is important to identify relevant background factors that have connection with behavioral, normative, and control beliefs and thereby have a better understanding of the determinants of organizational behaviors. Several organizational background factors that may indirectly influence organizational behaviors through behavioral, normative, and control beliefs include, but are not limited to, organizational incentives and support, leader-member relationship, team and group composition and climate, and organizational structure and culture.

Sixth, I found that attitude toward considerate voice measured from two different scales (i.e., bad-good and harmful-beneficial) loaded on two different factors. Because research have shown that the two components of attitude (i.e., instrumental and affective) may have differential relationships with important organizational and individual outcomes, the inclusion of measures that capture attitudes from both perspectives is important to fully understand the impact of attitude toward behavior on the actual behavior. Ajzen (2002) recommended that the instrumental component of attitude could be assessed by adjective pairs that are more cognitive in nature such as valuableinvaluable or harmful-beneficial while affective component of attitude could be captured by adjective pairs that are more experiential in nature such as pleasant-unpleasant or enjoyable- unenjoyable. 
Seventh, as discussed in the previous section regarding Hypothesis 3, one of the reasons for the non-significant relationship between normative expectancies and organizational behaviors could be the deficiency of the measure itself. More specifically, research has distinguished two different types of normative pressure: injunctive (i.e., perceptions of what others think one should do) and behavioral or descriptive (i.e., perceptions of what others are doing). I did not include the injunctive measure in the current study therefore the measure might not completely capture the normative expectancies. I agree with Ajzen (2002) that it is important to include both injunctive items and behavioral or descriptive items to have a complete measure of normative expectancies.

Eighth, the current study was the first attempt to develop behavioral engagement scale based on the conceptualization of Macey and Schneider (2008). Initial results suggested that there is a clear distinction between doing some more and doing something different for the organization. Except for self-identity expectancies, the distinction is documented in terms of the utilitarian and normative expectancies of behavioral engagement, attitude toward behavioral engagement, and behavioral engagement itself. However, the development of this measure is still at its initial stages, and further development and validation work is needed. I have suggested some enhancements that could improve the measure, one of which is creating items that further develop doing something different (i.e., proactive, adaptive and innovative behaviors) into separated dimensions. 
Finally, the current study highlights the importance of taking a cross-cultural perspective in organizational commitment research. Recent failures in generalizing the TCM into a different culture (e.g., Ko et al., 1997; Lee et al., 2001; Lee \& Yang, 2005) suggest that more work is needed to fully understand the nature of organizational commitment, how it is perceived, and how it influences organizational behaviors across different cultural contexts. Researchers could utilize the popular Hofstede's (1980) cultural framework in their investigation. Several cultural dimensions under this framework such as individualism/collectivism, power distance, or masculinity have been shown to have impacts on the relationships between organizational commitment and its outcomes. Moreover, recent developments in cross-cultural research such as cultural tightness-looseness (e.g., Gelfand, Nishii, \& Raver, 2006) could also be helpful in understanding the role of culture in the organizational and individual processes.

\section{Practical Implications}

Because none of hypotheses was supported, there is little basis for discussing the practical implications of the current study. However, as Fabrigar, Macdonald and Wegener (2005) suggested, "it is important to recognize that the extent to which an attitude predicts a behavior is not synonymous with the extent to which an attitude influences a behavior" (p. 105). Although organizational commitment does not usually predict specific behaviors, its influence on organizational and individual outcomes is undeniable. The target of organizational commitment goes beyond one's job position or work role (Hulin, 1991) and it incorporates a will to do good things for the organization's successes (Mowday, Porter \& Steers, 1982). Because of its tendency to act and go beyond the normal instrumental motivations of individuals, organizations should work 
hard to earn the organizational commitment of their employees (Buchanan, 1974; Eagly \& Chaiken, 1993; Scholl, 1981). Organizational commitment is a pledge of the employees and it "does not come cheap" (Solinger et al., 2008, p. 80).

\section{Limitations and Future Research}

I recognize several limitations of the current study that suggest the need for future research. The first limitation involves the use of specific organizational behavior as criteria for affective commitment. Following Solinger et al.'s (2008) recommendation, I chose the organizational behaviors for the current study to extend the range of organizational behaviors to be explained by organizational commitment. First, I aimed to choose behavioral criteria that are diverse in terms of their nature and characteristics (constructive vs. destructive and active vs. passive). Second, the behaviors must be important to organization. Third, I also tried to include organizational behaviors that had not been examined with organizational commitment to extend the literature. Because the TCM is regarded as the most dominant model in organizational commitment research and has attracted a large volume of studies (Bentein et al., 2005; Cohen, 2003, 2007;

Greenberg \& Baron, 2003), very few behaviors were qualified for the above criteria and most of them are very specific in nature. Future research testing the Eagly \& Chaiken's (1993) model should only include behaviors that are more general in nature to be more compatible with affective commitment. Harrison et al. (2006) illustrated the importance of compatibility between attitudes and behaviors by demonstrating that " a general set of actions at work - not specific behaviors - serves as the best criterion construct for overall job attitudes" (p.316). If researchers are interested in specific a behavior (which is 
often the case), attitude toward behavior should be measured. In such situations, they should not expect medium or high correlations between affective commitment and specific behaviors.

The second limitation is that supervisor ratings of considerate voice and behavioral engagement were not measured in term of their frequency in the current study. As noted, the behaviors in the current study might not be the usual approaches that respondent take to respond to organizational problems or events. Therefore, when the raters (i.e., supervisors) have little basis for rating a certain behavior because it is not usually engaged or observable, they may use their overall impression of the ratees or behaviors of similar quality that ratees often engage to assist them in rating the behavior under investigation (Sackett et al., 2006). For example, supervisors in the current study could base on the overall impression of the employee or loyalty - a usual approach to problematic organizational event - to rate considerate voice. Therefore, future research should include measure that can capture the frequency of the behaviors.

The third limitation involves the measurement of attitude toward behavior. More specifically, I only included items that capture the instrumental component (i.e., harmfulbeneficial) but not the affective component of attitude toward behavior in the hypotheses testing of the current study. The reason for me to exclude bad-good scale in the current study is because it tends to capture both components in one measure, which might contaminate the true relationship between different components of attitude and their outcomes (Ajzen, 2002). Future research should approach attitude toward behavior from 
two different perspectives to fully understand how attitude toward behavior influence organizational behaviors.

The fourth limitation is also related to a measurement issue. More specifically, I only included the behavioral or descriptive measure of normative expectancies in the current study. Research has generally distinguished the two types of normative pressures: injunctive (i.e., perceptions of what others think one should do) and behavioral or descriptive (i.e., perceptions of what others are doing) (Ajzen \& Fishbein, 2005). Therefore, future research should include items of both types to have a complete measure of normative expectancies.

The fifth limitation involves the use of self-report for all measures except organizational behaviors. Although I used supervisors-rated employee behaviors and incorporate a 3 month interval between two waves of data collection, it may still create common method bias, potentially threatening the validity of my conclusion about the relation between constructs (Podsakoff et al., 2003). However, as pointed out in the previous sections about the implication of the source of ratings, it is also a good idea to collect self-ratings report of the behaviors as there is a large discrepancy between selfratings and supervisor ratings. As noted, some of the behaviors such as CWBs and OCBs are more private, hence unobservable to supervisors (Dalal, 2005).

The sixth limitation is the restricted applicability of my model in predicting only volitional behaviors - that is, only behaviors that are performed consciously with a certain amount of cognitive deliberation (Eagly \& Chaiken, 1993; Fazio \& TowlesSchwen, 1999). Spontaneous, emotional, and/or reflexive behaviors are not suitable for 
this model (Solinger et al., 2008). However, I believe this limitation will not heavily threaten the usefulness of the model as most of the organizational behaviors of interest do have a certain amount of volition and consciousness (Harrison et al., 2006).

For future research, the discriminant validity of existing measures of affective commitment in the TCM can and should be improved by incorporating all three cognitive, emotional and behavioral components of organizational commitment as the affective commitment scale currently does not contain behavioral component (Solinger et al., 2008). In addition, the list of the behavioral expectancies discussed in this current study is important but no doubt incomplete. Future research should continue to base on the TRA framework to add additional background factors (e.g., individual and social processes) to improve the prediction and understanding of organizational behaviors. In conclusion, this current study provides some supports for Solinger et al.'s (2008) position that the TCM is not qualified to be a general model of organizational commitment, and thus contribute to the organizational commitment literature in several ways. First, the results suggested that affective commitment should be used as the core concept and sole indicator of employees' commitment to the organization. Second, the study provides some evidence about the role and benefits of various behavioral expectancies in understanding organizational behaviors. Third, I highlighted the role of contextual factors (e.g., national culture) and individual factors (e.g., perceived behavioral control) in understanding attitude - behavior relationship. Fourth, I have suggested several important recommendations to improve in the measurement of several constructs in the Eagly and Chaiken's (1993) model such as attitude toward behavior 
(i.e., affective vs. instrumental component), normative expectancies (i.e., injunctive vs. descriptive items), and the organizational behavior (i.e., frequency and source of ratings). Fifth, I developed the first behavioral engagement scale based of Macey and Schneider's (2008) conceptualization. Finally, the current study also brought organizational behavior research closer to the more general theory of human behavior (Solinger et al., 2008). 
Table 1. Inter-Item Correlations for Utilitarian Expectancies of Considerate Voice

\begin{tabular}{|c|c|c|c|c|c|c|c|c|c|c|c|c|c|}
\hline Item & Mean & SD & UECV1 & UECV2 & UECV3 & UECV4 & UECV5 & UECV6 & UECV7 & UECV8 & UECV9 & UECV10 & UECV11 \\
\hline UECV1 & 5.80 & 1.15 & & & & & & & & & & & \\
\hline UECV2 & 5.60 & 1.19 & $.62^{* *}$ & & & & & & & & & & \\
\hline UECV3 & 5.61 & 1.14 & $.65^{* *}$ & $.76^{* *}$ & & & & & & & & & \\
\hline UECV4 & 5.73 & 1.07 & $.63^{* *}$ & $.83^{* *}$ & $.77^{* *}$ & & & & & & & & \\
\hline UECV5 & 4.76 & 1.38 & $.25^{* *}$ & $.42^{* *}$ & $.38^{* *}$ & $.36^{* *}$ & & & & & & & \\
\hline UECV6 & 5.57 & 1.06 & $.55^{* *}$ & $.66^{* *}$ & $.72^{* *}$ & $.71^{* *}$ & $.43^{* *}$ & & & & & & \\
\hline UECV7 & 5.54 & 1.07 & $.57^{* * *}$ & $.70^{* *}$ & $.74^{* *}$ & $.72^{* *}$ & $.44^{* *}$ & $.83^{* *}$ & & & & & \\
\hline UECV8 & 5.48 & 1.07 & $.51^{* * *}$ & $.58^{* *}$ & $.66^{* *}$ & $.64^{* *}$ & $.36^{* *}$ & $.68^{* *}$ & $.74^{* *}$ & & & & \\
\hline UECV9 & 5.72 & 1.03 & $.61^{* *}$ & $.62^{* *}$ & $.67^{* *}$ & $.63^{* *}$ & $.29^{* *}$ & $.68^{* *}$ & $.68^{* *}$ & $.61^{* *}$ & & & \\
\hline UECV10 & 5.66 & 1.10 & $.50^{* *}$ & $.57^{* *}$ & $.64^{* *}$ & $.60^{* *}$ & $.31^{* *}$ & $.61^{* *}$ & $.59^{* *}$ & $.65^{* *}$ & $.70^{* *}$ & & \\
\hline UECV11 & 5.12 & 1.16 & $.43^{* *}$ & $.49^{* *}$ & $.51^{* *}$ & $.47^{* *}$ & $.45^{* *}$ & $.49^{* *}$ & $.53^{* *}$ & $.53^{* *}$ & $.44^{* *}$ & $.54^{* *}$ & \\
\hline
\end{tabular}


Table 2. Exploratory Factor Analysis for Utilitarian Expectancies of Considerate Voice

\begin{tabular}{|c|c|}
\hline Item & Factor 1 \\
\hline $\begin{array}{l}\text { UECV1. I believe that trying to come to an understanding with parties related } \\
\text { to the problem will yield good result for me }\end{array}$ & 0.71 \\
\hline $\begin{array}{l}\text { UECV2. I believe that, in collaboration with related parties in the } \\
\text { organization, trying to find a solution that is satisfactory to } \\
\text { everybody will bring good outcomes to me }\end{array}$ & 0.83 \\
\hline $\begin{array}{l}\text { UECV3. I believe that trying to work out an ideal solution in collaboration } \\
\text { with related parties will be good for me }\end{array}$ & 0.87 \\
\hline $\begin{array}{l}\text { UECV4. I believe that, together with related parties in the organization, } \\
\text { explore each other's opinions until the problems are solved will be } \\
\text { beneficial for me }\end{array}$ & 0.86 \\
\hline $\begin{array}{l}\text { UECV6. I believe that talking with related parties in the organization about } \\
\text { the problem until a total agreement is reached will yield good result } \\
\text { for me }\end{array}$ & 0.86 \\
\hline $\begin{array}{l}\text { UECV7. I believe that suggesting solutions to related parties in the } \\
\text { organization in dealing with organizational problems will bring } \\
\text { positive outcomes to me }\end{array}$ & 0.87 \\
\hline $\begin{array}{l}\text { UECV8. I believe that immediately reporting the problem to supervisor and } \\
\text { related parties in the organization will bring good outcomes to me }\end{array}$ & 0.78 \\
\hline $\begin{array}{l}\text { UECV9. I believe that immediately trying to find a solution will bring good } \\
\text { outcomes to me }\end{array}$ & 0.79 \\
\hline $\begin{array}{l}\text { UECV10. I believe that trying to think of different solutions to the problem } \\
\text { will be helpful for me }\end{array}$ & 0.73 \\
\hline
\end{tabular}

Note: $\mathrm{UECV}=$ Utilitarian expectancies of considerate voice. $\mathrm{N}=257$. Geomin rotation was used. 
Table 3. Inter-Item Correlations for Normative Expectancies of Considerate Voice

\begin{tabular}{|c|c|c|c|c|c|c|c|c|c|c|c|c|c|}
\hline Item & Mean & SD & NECV1 & NECV2 & NECV3 & NECV4 & NECV5 & NECV6 & NECV7 & NECV8 & NECV9 & NECV10 & NECV11 \\
\hline NECV1 & 5.55 & 1.12 & & & & & & & & & & & \\
\hline NECV2 & 5.58 & 1.18 & $.81^{* *}$ & & & & & & & & & & \\
\hline NECV3 & 5.57 & 1.13 & $.76^{* *}$ & $.83^{* *}$ & & & & & & & & & \\
\hline NECV4 & 5.46 & 1.18 & $.75^{* *}$ & $.76^{* *}$ & $.82^{* *}$ & & & & & & & & \\
\hline NECV5 & 4.65 & 1.30 & $.17^{* *}$ & $.16^{* *}$ & $.20^{* *}$ & $.19^{* *}$ & & & & & & & \\
\hline NECV6 & 5.28 & 1.17 & $.62^{* *}$ & $.65^{* *}$ & $.66^{* *}$ & $.70^{* *}$ & $.27^{* *}$ & & & & & & \\
\hline NECV7 & 5.42 & 1.05 & $.67^{* *}$ & $.67^{* *}$ & $.70^{* *}$ & $.69^{* *}$ & $.22^{* *}$ & $.70^{* *}$ & & & & & \\
\hline NECV8 & 5.51 & 1.17 & $.67^{* *}$ & $.66^{* *}$ & $.67^{* *}$ & $.70^{* *}$ & $.21^{* *}$ & $.65^{* *}$ & $.76^{* *}$ & & & & \\
\hline NECV9 & 5.53 & 1.09 & $.70^{* *}$ & $.70^{* *}$ & $.70^{* *}$ & $.68^{* *}$ & $.22^{* * *}$ & $.67^{* *}$ & $.73^{* *}$ & $.78^{* *}$ & & & \\
\hline NECV10 & 5.57 & 1.07 & $.68^{* *}$ & $.67^{* *}$ & $.68^{* *}$ & $.68^{* *}$ & $.21^{* *}$ & $.67^{* *}$ & $.70^{* *}$ & $.80^{* *}$ & $.83^{* *}$ & & \\
\hline NECV11 & 4.93 & 1.20 & $.38^{* *}$ & $.36^{* *}$ & $.35^{* *}$ & $.38^{* * *}$ & $.40^{* *}$ & $.38^{* *}$ & $.40^{* *}$ & $.42^{* *}$ & $.41^{* * *}$ & $.46^{* *}$ & \\
\hline
\end{tabular}

Note. $\mathrm{NECV}=$ Normative expectancies of considerate voice. $\mathrm{N}=253-256 . * * p<.01$. 
Table 4. Exploratory Factor Analysis for Normative Expectancies of Considerate Voice

\begin{tabular}{ll}
\hline \multicolumn{1}{c}{ Item } & Factor 1 \\
\hline NECV1. People around me at work (e.g., my supervisors and coworkers) & \\
usually try to come to an understanding with parties related to the & \\
problem & 0.85 \\
NECV2. People around me at work (e.g., my supervisors and coworkers) & \\
usually, in collaboration with related parties in the organization, try & \\
to find a solution that is satisfactory to everybody & 0.86 \\
NECV3. People around me at work (e.g., my supervisors and coworkers) \\
usually try to work out an ideal solution in collaboration with related \\
parities
\end{tabular}

Note $:$ NECV $=$ Normative expectancies of considerate voice. $\mathrm{N}=256$. Geomin rotation was used. 
Table 5. Inter-Item Correlations for Self-Identity Expectancies of Considerate Voice

\begin{tabular}{|c|c|c|c|c|c|c|c|c|c|c|c|c|c|}
\hline Item & Mean & SD & SECV1 & SECV2 & SECV3 & SECV4 & SECV5 & SECV6 & SECV7 & SECV8 & SECV9 & SECV10 & SECV11 \\
\hline SECV1 & 5.47 & 1.06 & & & & & & & & & & & \\
\hline SECV2 & 5.50 & 1.02 & $.73^{* *}$ & & & & & & & & & & \\
\hline SECV3 & 5.54 & 0.98 & $.79^{* *}$ & $.80^{* *}$ & & & & & & & & & \\
\hline SECV4 & 5.54 & 1.05 & $.70^{* *}$ & $.68^{* *}$ & $.79^{* *}$ & & & & & & & & \\
\hline SECV5 & 4.67 & 1.34 & $.31^{* *}$ & $.36^{* *}$ & $.33^{* *}$ & $.33^{* *}$ & & & & & & & \\
\hline SECV6 & 5.41 & 1.02 & $.66^{* *}$ & $.67^{* *}$ & $.71^{* *}$ & $.73^{* *}$ & $.38^{* *}$ & & & & & & \\
\hline SECV7 & 5.53 & 0.96 & $.71^{* *}$ & $.69^{* *}$ & $.76^{* *}$ & $.74^{* *}$ & $.31^{* * *}$ & $.80^{* *}$ & & & & & \\
\hline SECV8 & 5.40 & 1.04 & $.67^{* * *}$ & $.64^{* *}$ & $.69^{* *}$ & $.73^{* *}$ & $.33^{* * *}$ & $.71^{* *}$ & $.78^{* *}$ & & & & \\
\hline SECV9 & 5.69 & 0.98 & $.66^{* *}$ & $.64^{* *}$ & $.74^{* *}$ & $.67^{* *}$ & $.25^{* *}$ & $.62^{* *}$ & $.76^{* *}$ & $.73^{* *}$ & & & \\
\hline SECV10 & 5.59 & 1.00 & $.60^{* *}$ & $.59^{* *}$ & $.66^{* *}$ & $.63^{* *}$ & $25^{* *}$ & $.67^{* *}$ & $.72^{* *}$ & $.70^{* *}$ & $.83^{* *}$ & & \\
\hline SECV11 & 5.00 & 1.24 & $.45^{* * *}$ & $.48^{* * *}$ & $.44^{* *}$ & $.39^{* *}$ & $.52^{* *}$ & $.46^{* *}$ & $.44^{* *}$ & $.50^{* *}$ & $.45^{* *}$ & $.49^{* *}$ & \\
\hline
\end{tabular}

Note. SECV = Self-identity expectancies of considerate voice. $\mathrm{N}=252-256 .{ }^{* *} p<.01$. 
Table 6. Exploratory Factor Analysis for Self-Identity Expectancies of Considerate Voice

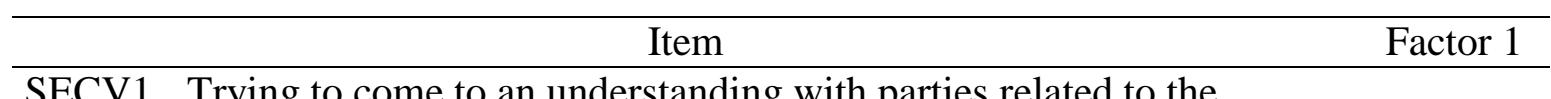

SECV1. Trying to come to an understanding with parties related to the problem makes me feel as a good member of the organization

SECV2. In collaboration with related parties in the organization, trying to find a solution that is satisfactory to everybody makes me feel as a good member of the organization

SECV3. Trying to work out an ideal solution in collaboration with related parities makes me feel as a good member of the organization

SECV4. Together with related parties in the organization, exploring each other's opinions until the problems are solved makes me feel as a good member of the organization

SECV6. Talking with related parties in the organization about the problem until a total agreement is reached makes me feel as a good member of the organization

SECV7. Suggesting solutions to related parties in the organization in dealing with organizational problems makes me feel as a good member of the organization

SECV8. Immediately reporting the problem to supervisor and related parties in the organization makes me feel as a good member of the organization

SECV9. Immediately trying to find a solution makes me feel as a good member of the organization

SECV10. Trying to think of different solutions to the problem makes me feel as a good member of the organization

Note. Self-identity expectancies of considerate voice. $\mathrm{N}=255$. Geomin rotation was used. 
Table 7. Inter-Item Correlations for Attitude toward Considerate Voice

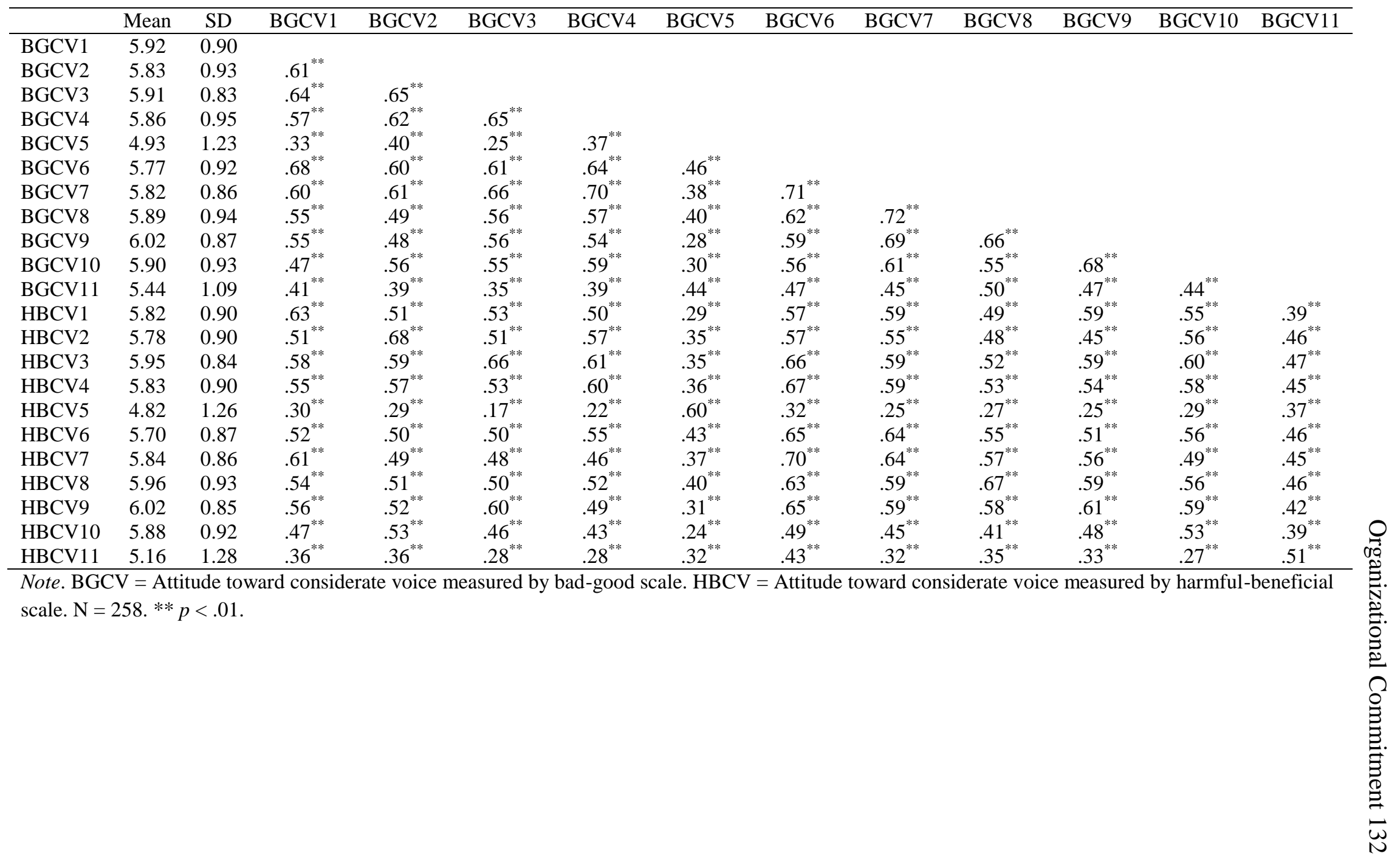


Table 7. Inter-Item Correlations for Attitude toward Considerate Voice (Continued)

\begin{tabular}{|c|c|c|c|c|c|c|c|c|c|c|c|c|c|}
\hline Item & Mean & SD & HBCV1 & HBCV2 & HBCV3 & HBCV4 & HBCV5 & HBCV6 & HBCV7 & HBCV8 & HBCV9 & HBCV10 & HBCV11 \\
\hline HBCV1 & 5.82 & 0.90 & & & & & & & & & & & \\
\hline HBCV2 & 5.78 & 0.90 & $.65^{* *}$ & & & & & & & & & & \\
\hline HBCV3 & 5.95 & 0.84 & $.69^{* * *}$ & $.69^{* * *}$ & & & & & & & & & \\
\hline HBCV4 & 5.83 & 0.90 & $.60^{* *}$ & $.67^{* *}$ & $.72^{* *}$ & & & & & & & & \\
\hline HBCV5 & 4.82 & 1.26 & $.31^{* *}$ & $.38^{* *}$ & $.33^{* * *}$ & $.36^{* *}$ & & & & & & & \\
\hline HBCV6 & 5.70 & 0.87 & $.61^{\text {*** }}$ & $.68^{* * *}$ & $.61^{* *}$ & $.68^{* *}$ & $.46^{* *}$ & & & & & & \\
\hline HBCV7 & 5.84 & 0.86 & $.66^{* *}$ & $.58^{* *}$ & $.68^{* * *}$ & $.63^{* *}$ & $.38^{* * *}$ & $.70^{* * *}$ & & & & & \\
\hline HBCV8 & 5.96 & 0.93 & $.62^{* *}$ & $.53^{* *}$ & $.65^{* *}$ & $.59^{9^{*}}$ & $.30^{* * *}$ & $.60^{* * *}$ & $.68^{* * *}$ & & & & \\
\hline HBCV9 & 6.02 & 0.85 & $.66^{* *}$ & $.56^{* *}$ & $.73^{* * *}$ & $.64^{* *}$ & $.27^{* *}$ & $.63^{* *}$ & $.71^{* *}$ & $.77^{* *}$ & & & \\
\hline HBCV10 & 5.88 & 0.92 & $.55^{* *}$ & $.51^{* *}$ & $.63^{* * *}$ & $.59^{* *}$ & $.21^{* *}$ & $.50^{* * *}$ & $.53^{* *}$ & $.54^{* * *}$ & $.63^{* *}$ & & \\
\hline HBCV11 & 5.16 & 1.28 & $.35^{* *}$ & $.38^{* *}$ & $.41^{* * *}$ & $.40^{* *}$ & $.36^{* *}$ & $.39^{* *}$ & $.47^{* *}$ & $.43^{* *}$ & $.41^{* *}$ & $.49^{* * *}$ & \\
\hline
\end{tabular}

Note. $\mathrm{HBCV}=$ Attitude toward considerate voice measured by harmful-beneficial scale. $\mathrm{N}=258 . * * p<.01$. 
Table 8. Exploratory Factor Analysis for Attitude toward Considerate Voice

\begin{tabular}{|c|c|c|}
\hline Item & Factor 1 & Factor 2 \\
\hline $\begin{array}{l}\text { BGCV1. Try to come to an understanding with parties related to } \\
\text { the problem }\end{array}$ & 0.52 & 0.25 \\
\hline $\begin{array}{l}\text { BGCV2. In collaboration with related parties in the organization, } \\
\text { try to find a solution that is satisfactory to everybody }\end{array}$ & 0.66 & 0.11 \\
\hline $\begin{array}{l}\text { BGCV3. Try to work out an ideal solution in collaboration with } \\
\text { related parities }\end{array}$ & 0.75 & 0.03 \\
\hline $\begin{array}{l}\text { BGCV4. Together with related parties in the organization, explore } \\
\text { each other's opinions until the problems are solved }\end{array}$ & 0.95 & -0.16 \\
\hline $\begin{array}{l}\text { BGCV6. Talk with related parties in the organization about the } \\
\text { problem until a total agreement is reached }\end{array}$ & 0.53 & 0.34 \\
\hline $\begin{array}{l}\text { BGCV7. Suggest solutions to related parties in the organization in } \\
\text { dealing with organizational problems }\end{array}$ & 0.87 & -0.01 \\
\hline $\begin{array}{l}\text { BGCV8. Immediately report the problem to supervisor and related } \\
\text { parties in the organization }\end{array}$ & 0.64 & 0.14 \\
\hline BGCV9. Immediately try to find a solution & 0.54 & 0.25 \\
\hline BGCV10. Try to think of different solutions to the problem & 0.50 & 0.27 \\
\hline $\begin{array}{l}\text { HBCV1. Try to come to an understanding with parties related to } \\
\text { the problem }\end{array}$ & 0.07 & 0.74 \\
\hline $\begin{array}{l}\text { HBCV2. In collaboration with related parties in the organization, } \\
\text { try to find a solution that is satisfactory to everybody }\end{array}$ & 0.25 & $\mathbf{0 . 5 3}$ \\
\hline $\begin{array}{l}\text { HBCV3. Try to work out an ideal solution in collaboration with } \\
\text { related parities }\end{array}$ & 0.14 & 0.73 \\
\hline $\begin{array}{l}\text { HBCV4. Together with related parties in the organization, explore } \\
\text { each other's opinions until the problems are solved }\end{array}$ & 0.21 & 0.61 \\
\hline $\begin{array}{l}\text { HBCV6. Talk with related parties in the organization about the } \\
\text { problem until a total agreement is reached }\end{array}$ & 0.21 & 0.60 \\
\hline $\begin{array}{l}\text { HBCV7. Suggest solutions to related parties in the organization in } \\
\text { dealing with organizational problems }\end{array}$ & -0.01 & 0.83 \\
\hline $\begin{array}{l}\text { HBCV8. Immediately report the problem to supervisor and related } \\
\text { parties in the organization }\end{array}$ & 0.05 & 0.76 \\
\hline HBCV9. Immediately try to find a solution & -0.08 & 0.92 \\
\hline HBCV10. Try to think of different solutions to the problem & -0.04 & 0.73 \\
\hline
\end{tabular}

Note. BGCV = Attitude toward considerate voice measured by bad-good scale. HBCV = Attitude toward considerate voice measured by harmful-beneficial scale. $\mathrm{N}=258$.

Geomin rotation was used. The correlation between the factors is .82 . 
Table 9. Inter-Item Correlations for Considerate Voice

\begin{tabular}{|c|c|c|c|c|c|c|c|c|c|c|c|c|c|}
\hline & Mean & SD & CV1 & CV2 & CV3 & CV4 & CV5 & CV6 & CV7 & CV8 & CV9 & CV10 & CV11 \\
\hline CV1 & 6.00 & 1.03 & & & & & & & & & & & \\
\hline CV2 & 5.89 & 1.07 & $.78^{* * *}$ & & & & & & & & & & \\
\hline CV3 & 5.91 & 1.00 & $.77^{* *}$ & $.82^{* *}$ & & & & & & & & & \\
\hline CV4 & 5.84 & 1.05 & $.71^{* *}$ & $.80^{* *}$ & $.82^{* *}$ & & & & & & & & \\
\hline CV5 & 5.21 & 1.31 & $.28^{* * *}$ & $.35^{* *}$ & $.31^{* *}$ & $.37^{* * *}$ & & & & & & & \\
\hline CV6 & 5.75 & 0.91 & $.56^{* *}$ & $.58^{* *}$ & $.62^{* *}$ & $.69^{* *}$ & $.51^{* *}$ & & & & & & \\
\hline CV7 & 5.75 & 0.93 & $.62^{* *}$ & $.66^{* *}$ & $.68^{* *}$ & $.71^{* *}$ & $.43^{* *}$ & $.74^{* *}$ & & & & & \\
\hline CV8 & 5.95 & 0.99 & $.69^{* *}$ & $.64^{* *}$ & $.63^{* *}$ & $.59^{* *}$ & $.18^{* *}$ & $.56^{* *}$ & $.66^{* *}$ & & & & \\
\hline CV9 & 5.82 & 0.89 & $.52^{* *}$ & $.60^{* *}$ & $.53^{* *}$ & $.59^{* *}$ & $.16^{*}$ & $.51^{* *}$ & $.61^{* *}$ & $.65^{* *}$ & & & \\
\hline CV10 & 5.75 & 0.99 & $.40^{* *}$ & $.57^{* *}$ & $.47^{* *}$ & $.55^{* *}$ & $.14^{*}$ & $.41^{* *}$ & $.50^{* *}$ & $.50^{* *}$ & $.76^{* *}$ & & \\
\hline CV11 & 5.23 & 1.06 & $.48^{* *}$ & $.51^{* *}$ & $.42^{* *}$ & $.40^{* * *}$ & $.21^{* *}$ & $.41^{* *}$ & $.41^{* *}$ & $.41^{* * *}$ & $.50^{* * *}$ & $.59^{* *}$ & \\
\hline
\end{tabular}

Note. $\mathrm{CV}=$ Considerate voice. $\mathrm{N}=257$. $* * p<.01$. 
Table 10. Exploratory Factor Analysis for Considerate Voice

\begin{tabular}{|c|c|c|c|}
\hline & Item & Factor 1 & Factor 2 \\
\hline CV1. & $\begin{array}{l}\text { Try to come to an understanding with parties related to } \\
\text { the problem }\end{array}$ & 0.91 & -0.10 \\
\hline CV2. & $\begin{array}{l}\text { In collaboration with related parties in the organization, } \\
\text { try to find a solution that is satisfactory to everybody }\end{array}$ & 0.86 & 0.05 \\
\hline CV3. & $\begin{array}{l}\text { Try to work out an ideal solution in collaboration with } \\
\text { related parities }\end{array}$ & 1.00 & -0.14 \\
\hline CV4. & $\begin{array}{l}\text { Together with related parties in the organization, explore } \\
\text { each other's opinions until the problems are solved }\end{array}$ & 0.88 & 0.01 \\
\hline CV5. & $\begin{array}{l}\text { Try to compromise with related parties in the } \\
\text { organization in dealing with organizational problems }\end{array}$ & 0.51 & -0.17 \\
\hline CV6. & $\begin{array}{l}\text { Talk with related parties in the organization about the } \\
\text { problem until a total agreement is reached }\end{array}$ & 0.70 & 0.04 \\
\hline CV7. & $\begin{array}{l}\text { Suggest solutions to related parties in the organization in } \\
\text { dealing with organizational problems }\end{array}$ & 0.69 & 0.15 \\
\hline CV8. & $\begin{array}{l}\text { Immediately report the problem to supervisor and related } \\
\text { parties in the organization }\end{array}$ & 0.55 & 0.26 \\
\hline CV9. & Immediately try to find a solution & 0.13 & 0.79 \\
\hline CV10. & Try to think of different solutions to the problem & -0.01 & 0.88 \\
\hline CV11. & Ask related parties in the organization for compromises & 0.20 & 0.48 \\
\hline
\end{tabular}

Note: $\mathrm{CV}=$ Considerate voice. $\mathrm{N}=257$. Geomin rotation was used. The correlation between factors is .67 . 
Table 11. Confirmatory Factor Analysis Results for Scales involved Considerate Voice

\begin{tabular}{|c|c|c|c|c|c|c|c|c|}
\hline Model & $\mathrm{N}$ & $X^{2}$ & df & CFI & RMSEA & SRMR & $\Delta \mathrm{X}^{2}$ & $\Delta \mathrm{df}$ \\
\hline \multicolumn{9}{|l|}{ Utilitarian expectancies of considerate voice } \\
\hline One-factor model & 257 & 219.86 & 44 & 0.92 & 0.125 & 0.042 & & \\
\hline One-factor model (without Item 5 and 11) & 257 & 172.00 & 27 & 0.92 & 0.145 & 0.038 & $47.86 * *$ & 17 \\
\hline \multicolumn{9}{|l|}{ Normative expectancies of considerate voice } \\
\hline One-factor model & 257 & 264.38 & 44 & 0.91 & 0.14 & 0.05 & & \\
\hline One-factor model (without Item 5 and 11) & 256 & 219.93 & 27 & 0.91 & 0.167 & 0.041 & $44.45^{* *}$ & 17 \\
\hline \multicolumn{9}{|l|}{ Self-identity expectancies of considerate voice } \\
\hline One-factor model & 255 & 295.76 & 44 & 0.90 & 0.15 & 0.051 & & \\
\hline One-factor model (without Item 5 and 11) & 255 & 215.99 & 27 & 0.92 & 0.166 & 0.038 & $79.77 * *$ & 17 \\
\hline \multicolumn{9}{|l|}{ Attitude toward considerate voice } \\
\hline One-factor model & 258 & 955.92 & 209 & 0.83 & 0.118 & 0.06 & & \\
\hline Two-factor model & 258 & 813.19 & 208 & 0.86 & 0.106 & 0.056 & $142.74 * *$ & 1 \\
\hline Two-factor model (without Item 5 and 11) & 258 & 498.44 & 133 & 0.90 & 0.103 & 0.042 & $314.75^{* *}$ & 75 \\
\hline One-factor model (Harmful-Beneficial items, without Item 5 and 11) & 258 & 127.60 & 27 & 0.94 & 0.12 & 0.035 & $370.84 * *$ & 106 \\
\hline \multicolumn{9}{|l|}{ Considerate voice } \\
\hline Two-factor model & 257 & 296.86 & 43 & 0.88 & 0.152 & 0.059 & & \\
\hline One-factor model (without Item 5, 9, 10, and 11) & 257 & 121.00 & 14 & 0.93 & 0.172 & 0.04 & $175.86^{* *}$ & 29 \\
\hline
\end{tabular}

Note: $* * \mathrm{p}<.01$. 
Table 12. Exploratory Factor Analysis Results for the Final Scales involved Considerate Voice

\begin{tabular}{|c|c|c|c|c|c|c|}
\hline & Item & Factor 1 & Factor 2 & Factor 3 & Factor 4 & Factor 5 \\
\hline UECV1. & $\begin{array}{l}\text { I believe that trying to come to an understanding with parties related to the } \\
\text { problem will yield good result for me }\end{array}$ & 0.61 & 0.10 & 0.03 & 0.01 & -0.01 \\
\hline UECV2. & $\begin{array}{l}\text { I believe that, in collaboration with related parties in the organization, trying to } \\
\text { find a solution that is satisfactory to everybody will bring good outcomes to me }\end{array}$ & 0.87 & 0.04 & -0.08 & 0.04 & -0.02 \\
\hline UECV3. & $\begin{array}{l}\text { I believe that trying to work out an ideal solution in collaboration with related } \\
\text { parties will be good for me }\end{array}$ & 0.84 & 0.04 & 0.01 & -0.02 & 0.05 \\
\hline UECV4. & $\begin{array}{l}\text { I believe that, together with related parties in the organization, explore each } \\
\text { other's opinions until the problems are solved will be beneficial for me }\end{array}$ & 0.83 & -0.02 & 0.07 & 0.02 & 0.03 \\
\hline UECV6. & $\begin{array}{l}\text { I believe that talking with related parties in the organization about the problem } \\
\text { until a total agreement is reached will yield good result for me }\end{array}$ & 0.71 & -0.02 & 0.20 & 0.02 & -0.04 \\
\hline UECV7. & $\begin{array}{l}\text { I believe that suggesting solutions to related parties in the organization in dealing } \\
\text { with organizational problems will bring positive outcomes to me }\end{array}$ & 0.88 & -0.01 & 0.01 & -0.04 & 0.01 \\
\hline UECV8. & $\begin{array}{l}\text { I believe that immediately reporting the problem to supervisor and related parties } \\
\text { in the organization will bring good outcomes to me }\end{array}$ & 0.66 & 0.07 & 0.07 & 0.00 & 0.00 \\
\hline NECV1. & $\begin{array}{l}\text { People around me at work (e.g., my supervisors and coworkers) usually try to } \\
\text { come to an understanding with parties related to the problem }\end{array}$ & 0.07 & 0.80 & 0.02 & -0.01 & 0.05 \\
\hline NECV2. & $\begin{array}{l}\text { People around me at work (e.g., my supervisors and coworkers) usually, in } \\
\text { collaboration with related parties in the organization, try to find a solution that is } \\
\text { satisfactory to everybody }\end{array}$ & 0.04 & 0.87 & -0.01 & 0.03 & 0.00 \\
\hline NECV3. & $\begin{array}{l}\text { People around me at work (e.g., my supervisors and coworkers) usually try to } \\
\text { work out an ideal solution in collaboration with related parities }\end{array}$ & 0.00 & 0.94 & -0.03 & -0.02 & 0.05 \\
\hline NECV4. & $\begin{array}{l}\text { People around me at work (e.g., my supervisors and coworkers) usually, together } \\
\text { with related parties in the organization, explore each other's opinions until the } \\
\text { problems are solved }\end{array}$ & -0.01 & 0.85 & 0.05 & 0.02 & 0.01 \\
\hline NECV6. & $\begin{array}{l}\text { People around me at work (e.g., my supervisors and coworkers) usually talk with } \\
\text { related parties in the organization about the problem until a total agreement is } \\
\text { reached }\end{array}$ & -0.05 & 0.66 & 0.18 & 0.00 & -0.04 \\
\hline NECV7. & $\begin{array}{l}\text { People around me at work (e.g., my supervisors and coworkers) usually suggest } \\
\text { solutions to related parties in the organization in dealing with organizational } \\
\text { problems }\end{array}$ & 0.06 & 0.55 & 0.27 & -0.01 & -0.05 \\
\hline NECV8. & $\begin{array}{l}\text { People around me at work (e.g., my supervisors and coworkers) usually report the } \\
\text { problem to supervisor and related parties in the organization immediately }\end{array}$ & 0.09 & 0.53 & 0.22 & 0.05 & -0.02 \\
\hline
\end{tabular}


Table 12. Exploratory Factor Analysis Results for Considerate Voice-Related Final Scales (Continued)

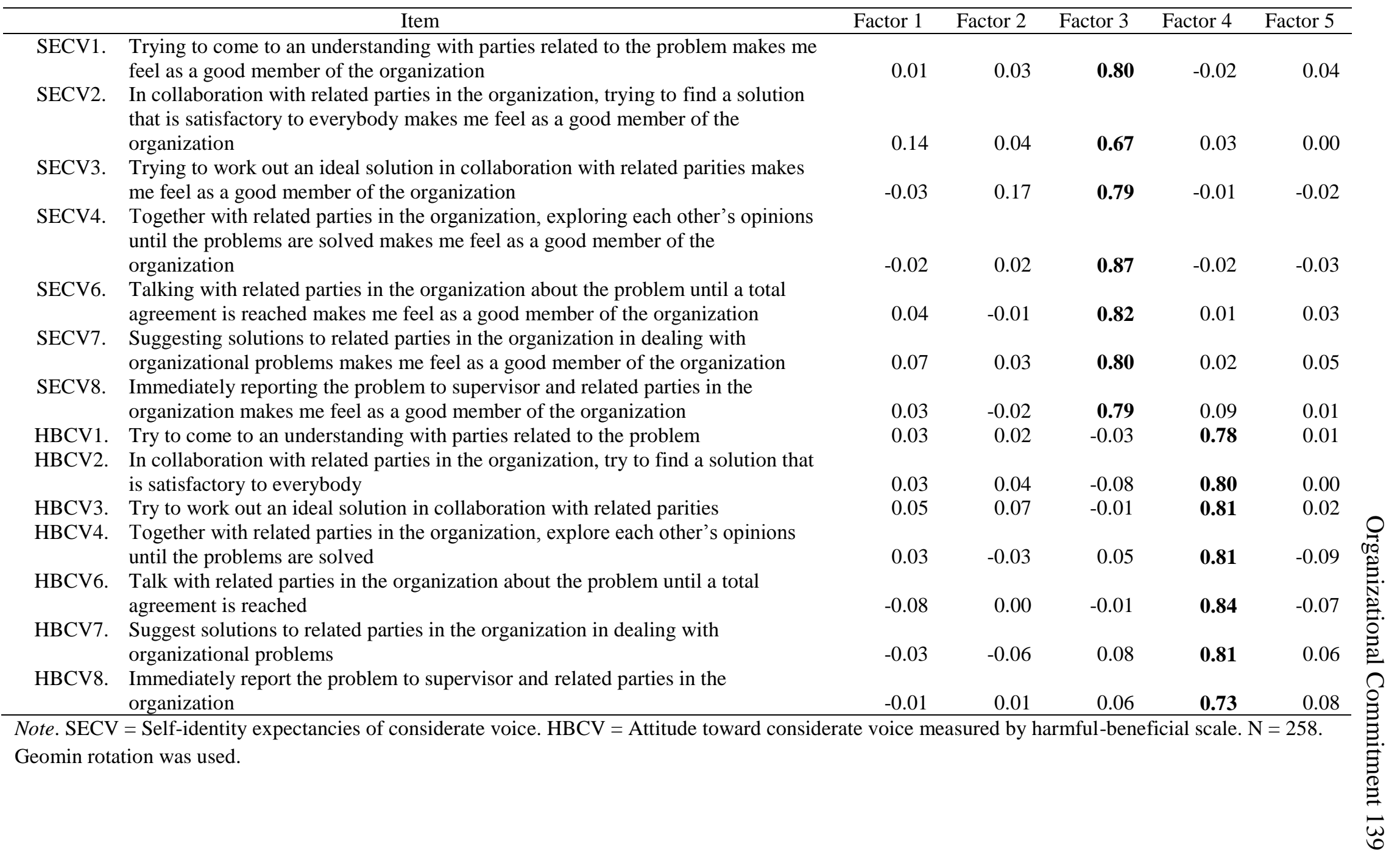


Table 12. Exploratory Factor Analysis Results for Considerate Voice-Related Final Scales (Continued)

\begin{tabular}{|c|c|c|c|c|c|c|}
\hline & Item & Factor 1 & Factor 2 & Factor 3 & Factor 4 & Factor 5 \\
\hline CV1. & Try to come to an understanding with parties related to the problem & -0.02 & 0.04 & -0.04 & 0.06 & 0.84 \\
\hline CV2. & $\begin{array}{l}\text { In collaboration with related parties in the organization, try to find a } \\
\text { solution that is satisfactory to everybody }\end{array}$ & -0.08 & 0.05 & 0.07 & -0.03 & 0.89 \\
\hline CV3. & Try to work out an ideal solution in collaboration with related parities & 0.01 & 0.08 & -0.02 & 0.03 & 0.90 \\
\hline CV4. & $\begin{array}{l}\text { Together with related parties in the organization, explore each other's } \\
\text { opinions until the problems are solved }\end{array}$ & 0.00 & -0.02 & 0.00 & 0.00 & 0.89 \\
\hline CV6. & $\begin{array}{l}\text { Talk with related parties in the organization about the problem until a } \\
\text { total agreement is reached }\end{array}$ & 0.06 & -0.05 & 0.01 & 0.02 & 0.71 \\
\hline CV7. & $\begin{array}{l}\text { Suggest solutions to related parties in the organization in dealing with } \\
\text { organizational problems }\end{array}$ & 0.10 & -0.11 & -0.04 & 0.00 & 0.79 \\
\hline CV8. & $\begin{array}{l}\text { Immediately report the problem to supervisor and related parties in the } \\
\text { organization }\end{array}$ & -0.02 & -0.04 & 0.07 & -0.05 & 0.73 \\
\hline
\end{tabular}

Note. $\mathrm{CV}=$ Considerate voice. $\mathrm{N}=258$. Geomin rotation was used. 
Organizational Commitment 141

Table 13. Inter-Item Correlations for Utilitarian Expectancies of Production Deviance

\begin{tabular}{lccccccc}
\hline & Mean & SD & UEPD1 & UEPD2 & UEPD3 & UEPD4 & UEPD5 \\
\hline UEPD1 & 1.93 & 1.23 & & & & & \\
UEPD2 & 2.33 & 1.43 & $.59^{* *}$ & & & \\
UEPD3 & 2.02 & 1.29 & $.75^{* *}$ & $.67^{* *}$ & & \\
UEPD4 & 2.18 & 1.41 & $.58^{* *}$ & $.61^{* *}$ & $.80^{* *}$ & \\
UEPD5 & 2.25 & 1.42 & $.59^{* *}$ & $.56^{* *}$ & $.72^{* *}$ & $.67^{* *}$ & \\
\hline Note. UEPD & = Utilitarian expectancies of production deviance. $\mathrm{N}=244-247 . *^{* *} p<.01$.
\end{tabular}


Table 14. Exploratory Factor Analysis for Utilitarian Expectancies of Production Deviance

\begin{tabular}{|c|c|}
\hline Item & Factor 1 \\
\hline $\begin{array}{l}\text { UEPD1. I believe that sometimes doing my work incorrectly on purpose } \\
\text { will bring good outcomes to me. }\end{array}$ & 0.77 \\
\hline $\begin{array}{l}\text { UEPD2. I believe that sometimes working slowly on purpose when things } \\
\text { need to get done will yield good result for me. }\end{array}$ & 0.72 \\
\hline $\begin{array}{l}\text { UEPD3. I believe that sometimes failing to follow instructions on purpose } \\
\text { will be good for me. }\end{array}$ & 0.95 \\
\hline $\begin{array}{l}\text { UEPD4. I believe that sometimes giving obscure instructions or information } \\
\text { to others will be good for me }\end{array}$ & 0.84 \\
\hline $\begin{array}{l}\text { UEPD5. I believe that sometimes not providing warning when I know } \\
\text { things can go wrong later will be beneficial for me }\end{array}$ & 0.77 \\
\hline
\end{tabular}
Note. UEPD = Utilitarian expectancies of production deviance. $\mathrm{N}=248$. Geomin rotation was used. 
Organizational Commitment 143

Table 15. Inter-Item Correlations for Normative Expectancies of Production Deviance

\begin{tabular}{lccccccc}
\hline & Mean & SD & NEPD1 & NEPD2 & NEPD3 & NEPD4 & NEPD5 \\
\hline NEPD1 & 2.41 & 1.43 & & & & & \\
NEPD2 & 2.64 & 1.60 & $.80^{* *}$ & & & \\
NEPD3 & 2.49 & 1.53 & $.85^{* *}$ & $.85^{* *}$ & & \\
NEPD4 & 2.50 & 1.47 & $.82^{* *}$ & $.82^{* *}$ & $.90^{* *}$ & \\
NEPD5 & 2.60 & 1.54 & $.85^{* *}$ & $.84^{* *}$ & $.85^{* *}$ & $.85^{* *}$ & \\
\hline Note. NEPD = Normative expectancies of production deviance. $\mathrm{N}=255-256 .{ }^{* *} p<.01$.
\end{tabular}


Table 16. Exploratory Factor Analysis for Normative Expectancies of Production Deviance

\begin{tabular}{|c|c|c|}
\hline & Item & Factor 1 \\
\hline NEPD1. & $\begin{array}{l}\text { People around me at work (e.g., my supervisors and coworkers) } \\
\text { sometimes do their work incorrectly on purpose }\end{array}$ & 0.90 \\
\hline NEPD2. & $\begin{array}{l}\text { People around me at work (e.g., my supervisors and coworkers) } \\
\text { sometimes work slowly on purpose when things need to get done }\end{array}$ & 0.89 \\
\hline NEPD3. & $\begin{array}{l}\text { People around me at work (e.g., my supervisors and coworkers) } \\
\text { sometimes fail to follow instructions on purpose }\end{array}$ & 0.95 \\
\hline NEPD4. & $\begin{array}{l}\text { People around me at work (e.g., my supervisors and coworkers) } \\
\text { sometimes give obscure instructions or information to others }\end{array}$ & 0.93 \\
\hline NEPD5. & $\begin{array}{l}\text { People around me at work (e.g., my supervisors and coworkers) } \\
\text { sometimes do not provide warning when they know things can go } \\
\text { wrong later }\end{array}$ & 0.91 \\
\hline
\end{tabular}

Note . UEPD $=$ Normative expectancies of production deviance. $\mathrm{N}=256$. Geomin rotation was used. 
Organizational Commitment 145

Table 17. Inter-Item Correlations for Self-Identity Expectancies of Production Deviance

\begin{tabular}{lccccccc}
\hline & Mean & SD & SEPD1 & SEPD2 & SEPD3 & SEPD4 & SEPD5 \\
\hline SEPD1 & 3.95 & 2.07 & & & & & \\
SEPD2 & 3.93 & 2.06 & $.94^{* *}$ & & & & \\
SEPD3 & 3.99 & 2.09 & $.96^{* *}$ & $.97^{* *}$ & & & \\
SEPD4 & 3.98 & 2.06 & $.94^{* *}$ & $.95^{* *}$ & $.97^{* *}$ & & \\
SEPD5 & 3.97 & 1.99 & $.91^{* *}$ & $.92^{* *}$ & $.93^{* *}$ & $.96^{* *}$ & \\
\hline
\end{tabular}

Note. SEPD = Self-identity expectancies of production deviance.

$\mathrm{N}=251-252 . * * p<.01$. 
Table 18. Exploratory Factor Analysis for Self-Identity Expectancies of Production Deviance

\begin{tabular}{clc}
\hline \multicolumn{1}{c}{ Item } & Factor 1 \\
\hline SEPD1. Purposely doing your work incorrectly makes me feel as a bad & 0.97 \\
member of the organization & 0.97 \\
SEPD2. $\begin{array}{l}\text { Purposely working slowly when things need to get done makes me } \\
\text { feel as a bad member of the organization }\end{array}$ & 0.99 \\
$\begin{array}{l}\text { SEPD4. } \begin{array}{l}\text { Giving obscure instructions or information to others makes me feel } \\
\text { as a bad member of the organization }\end{array} \\
\text { SEPD5. Not providing warning when you know things can go wrong later } \\
\text { makes me feel as a bad member of the organization }\end{array}$ & 0.98 \\
\hline
\end{tabular}

Note. SEPD = Self-identity expectancies of production deviance. $\mathrm{N}=252$. Geomin rotation was used. 
Table 19. Inter-Item Correlations for Attitude toward Production Deviance

\begin{tabular}{|c|c|c|c|c|c|c|c|c|c|c|c|c|}
\hline & Mean & $\mathrm{SD}$ & BGPD1 & BGPD2 & BGPD3 & BGPD4 & BGPD5 & HBPD1 & HBPD2 & HBPD3 & HBPD4 & HBPD5 \\
\hline BGPD1 & 1.86 & 1.24 & & & & & & & & & & \\
\hline BGPD2 & 1.91 & 1.25 & $.85^{* *}$ & & & & & & & & & \\
\hline BGPD3 & 1.87 & 1.41 & $.81^{* *}$ & $.82^{* *}$ & & & & & & & & \\
\hline BGPD4 & 1.92 & 1.32 & $.85^{\text {** }}$ & $.86^{* * *}$ & $.79^{* *}$ & & & & & & & \\
\hline BGPD5 & 2.17 & 1.33 & $.77^{* *}$ & $.77^{* *}$ & $.70^{* *}$ & $.82^{* *}$ & & & & & & \\
\hline HBPD1 & 2.04 & 1.35 & $.74^{* *}$ & $.75^{* *}$ & $69^{* *}$ & $.73^{* *}$ & $.69^{* *}$ & & & & & \\
\hline HBPD2 & 2.07 & 1.29 & $.75^{* *}$ & $.78^{* *}$ & $.70^{* *}$ & $.75^{* *}$ & $.70^{* *}$ & $.91^{\text {** }}$ & & & & \\
\hline HBPD3 & 1.98 & 1.36 & $.76^{* * *}$ & $.78^{* *}$ & $.73^{* *}$ & $.78^{* *}$ & $.72^{* *}$ & $.93^{* *}$ & $.93^{* *}$ & & & \\
\hline HBPD4 & 2.12 & 1.43 & $.68^{* *}$ & $.69^{* *}$ & $.64^{* *}$ & $.72^{* *}$ & $.65^{* *}$ & $.86^{* *}$ & $.85^{* *}$ & $.88^{* *}$ & & \\
\hline HBPD5 & 2.43 & 1.51 & $.58^{* *}$ & $.57^{* *}$ & $.53^{* *}$ & $.57^{* *}$ & $.66^{* *}$ & $.74^{* *}$ & $.70^{* *}$ & $.72^{* *}$ & $.75^{* *}$ & \\
\hline
\end{tabular}

Note. BGPD = Attitude toward production deviance measured by bad-good scale. HBPD = Attitude toward production deviance measured by harmful-beneficial scale. $\mathrm{N}=258$. $* * p<.01$. 
Table 20. Exploratory Factor Analysis for Attitude toward Production Deviance

\begin{tabular}{lr}
\hline \multicolumn{1}{c}{ Item } & Factor 1 \\
\hline BGPD1. Purposely do your work incorrectly & 0.83 \\
BGPD2. Purposely work slowly when things need to get done & 0.84 \\
BGPD3. Purposely fail to follow instructions & 0.78 \\
BGPD4. Give obscure instructions or information to others & 0.84 \\
BGPD5. Do not provide warning when you know things can go wrong later & 0.78 \\
HBPD1. Purposely do your work incorrectly & 0.94 \\
HBPD2. Purposely work slowly when things need to get done & 0.94 \\
HBPD3. Purposely fail to follow instructions & 0.97 \\
HBPD4. Give obscure instructions or information to others & 0.89 \\
HBPD5. Do not provide warning when you know things can go wrong later & 0.75 \\
\hline
\end{tabular}
Note. BGPD = Attitude toward production deviance measured by bad-good scale. HBPD $=$ Attitude toward production deviance measured by harmful-beneficial scale. $\mathrm{N}=258$. Geomin rotation was used. 
Organizational Commitment 149

Table 21. Inter-Item Correlations for Production Deviance

\begin{tabular}{lccccccc}
\hline & Mean & SD & PD1 & PD2 & PD3 & PD4 & PD5 \\
\hline PD1 & 1.05 & 0.25 & & & & & \\
PD2 & 1.18 & 0.48 & $.36^{* *}$ & & & & \\
PD3 & 1.07 & 0.29 & $.55^{* *}$ & $.38^{* *}$ & & & \\
PD4 & 1.11 & 0.36 & $.39^{* *}$ & $.41^{* *}$ & $.56^{* *}$ & & \\
PD5 & 1.27 & 0.47 & $.16^{* *}$ & .10 & $.14^{*}$ & $.24^{* *}$ & \\
\hline
\end{tabular}

Note. $\mathrm{PD}=$ Production deviance. $\mathrm{N}=257 . * \mathrm{p}<.05 . * * \mathrm{p}<.01$. 
Organizational Commitment 150

Table 22. Exploratory Factor Analysis for Production Deviance

\begin{tabular}{lr}
\hline \multicolumn{1}{c}{ Item } & Factor 1 \\
\hline PD1. Purposely do your work incorrectly & $\mathbf{0 . 6 5}$ \\
PD2. Purposely work slowly when things need to get done & $\mathbf{0 . 5 2}$ \\
PD3. Purposely fail to follow instructions & $\mathbf{0 . 8 1}$ \\
PD4. Give obscure instructions or information to others & $\mathbf{0 . 6 9}$ \\
PD5. Do not provide warning when you know things can go wrong later & 0.23 \\
\hline
\end{tabular}

Note. $\mathrm{PD}=$ Production deviance. $\mathrm{N}=257$. Geomin rotation was used. 
Table 23. Confirmatory Factor Analysis Results for Scales involved Production Deviance

\begin{tabular}{|c|c|c|c|c|c|c|c|c|}
\hline Model & $\mathrm{N}$ & $\mathrm{X}^{2}$ & $\mathrm{df}$ & CFI & RMSEA & SRMR & $\Delta X^{2}$ & $\Delta \mathrm{df}$ \\
\hline \multicolumn{9}{|c|}{ Utilitarian expectancies of production deviance } \\
\hline One factor model & 248 & 14.85 & 5 & 0.99 & 0.089 & 0.018 & & \\
\hline \multicolumn{9}{|c|}{ Normative expectancies of production deviance } \\
\hline One factor model & 256 & 23.42 & 5 & 0.99 & 0.120 & 0.010 & & \\
\hline \multicolumn{9}{|c|}{ Self-identity expectancies of production deviance } \\
\hline One factor model & 252 & 60.12 & 5 & 0.98 & 0.209 & 0.007 & & \\
\hline \multicolumn{9}{|l|}{ Attitude toward production deviance } \\
\hline One factor model & 258 & 22.05 & 5 & 0.99 & 0.12 & 0.02 & & \\
\hline \multicolumn{9}{|l|}{ Production deviance } \\
\hline One factor model & 257 & 14.47 & 5 & 0.97 & 0.09 & 0.03 & & \\
\hline One factor model (without item 5) & 257 & 8.04 & 2 & 0.98 & 0.11 & 0.03 & $6.42^{n s}$ & 3 \\
\hline
\end{tabular}

Note. $n s=$ non-significant 
Table 24. Exploratory Factor Analysis for the Final Scales involved Production Deviance

\begin{tabular}{|c|c|c|c|c|c|c|}
\hline & Item & $\begin{array}{l}\text { Factor } \\
1\end{array}$ & $\begin{array}{l}\text { Factor } \\
2\end{array}$ & $\begin{array}{l}\text { Factor } \\
3\end{array}$ & $\begin{array}{l}\text { Factor } \\
4\end{array}$ & $\begin{array}{c}\text { Factor } \\
5\end{array}$ \\
\hline UEPD1. & $\begin{array}{l}\text { I believe that sometimes doing my work incorrectly on purpose will bring good outcomes to } \\
\text { me. }\end{array}$ & 0.72 & 0.09 & 0.00 & -0.03 & 0.06 \\
\hline UEPD2. & $\begin{array}{l}\text { I believe that sometimes working slowly on purpose when things need to get done will yield } \\
\text { good result for me. }\end{array}$ & 0.66 & 0.06 & -0.01 & 0.02 & 0.02 \\
\hline UEPD3. & I believe that sometimes failing to follow instructions on purpose will be good for me. & 1.01 & -0.06 & 0.00 & 0.01 & 0.00 \\
\hline UEPD4. & $\begin{array}{l}\text { I believe that sometimes giving obscure instructions or information to others will be good for } \\
\text { me }\end{array}$ & 0.81 & 0.03 & 0.00 & 0.00 & -0.08 \\
\hline NEPD1. & $\begin{array}{l}\text { People around me at work (e.g., my supervisors and coworkers) sometimes do their work } \\
\text { incorrectly on purpose }\end{array}$ & 0.02 & 0.88 & 0.01 & -0.04 & 0.04 \\
\hline NEPD2. & $\begin{array}{l}\text { People around me at work (e.g., my supervisors and coworkers) sometimes work slowly on } \\
\text { purpose when things need to get done }\end{array}$ & 0.02 & 0.87 & 0.03 & 0.06 & -0.05 \\
\hline NEPD3. & $\begin{array}{l}\text { People around me at work (e.g., my supervisors and coworkers) sometimes fail to follow } \\
\text { instructions on purpose }\end{array}$ & 0.05 & 0.94 & -0.01 & -0.01 & 0.02 \\
\hline NEPD4. & $\begin{array}{l}\text { People around me at work (e.g., my supervisors and coworkers) sometimes give obscure } \\
\text { instructions or information to others }\end{array}$ & -0.03 & 0.95 & -0.02 & 0.00 & -0.01 \\
\hline SEPD1. & Purposely doing your work incorrectly makes me feel as a bad member of the organization & 0.01 & -0.01 & 0.97 & 0.01 & 0.01 \\
\hline SEPD2. & $\begin{array}{l}\text { Purposely working slowly when things need to get done makes me feel as a bad member of } \\
\text { the organization }\end{array}$ & 0.01 & -0.01 & 0.97 & -0.02 & 0.00 \\
\hline SEPD3. & Purposely failing to follow instructions makes me feel as a bad member of the organization & -0.03 & 0.02 & 0.99 & 0.00 & -0.01 \\
\hline SEPD4. & $\begin{array}{l}\text { Giving obscure instructions or information to others makes me feel as a bad member of the } \\
\text { organization }\end{array}$ & 0.00 & 0.01 & 0.97 & 0.00 & 0.00 \\
\hline HBPD1. & Purposely do your work incorrectly & 0.02 & 0.00 & 0.00 & 0.95 & 0.00 \\
\hline HBPD2. & Purposely work slowly when things need to get done & 0.01 & 0.00 & -0.01 & 0.94 & 0.04 \\
\hline HBPD3. & Purposely fail to follow instructions & 0.01 & 0.01 & -0.01 & 0.97 & 0.02 \\
\hline HBPD4. & Give obscure instructions or information to others & -0.04 & 0.00 & 0.01 & 0.92 & -0.04 \\
\hline PD1. & Purposely do your work incorrectly & -0.07 & 0.01 & -0.08 & -0.07 & 0.67 \\
\hline PD2. & Purposely work slowly when things need to get done & 0.12 & -0.07 & 0.08 & -0.03 & 0.51 \\
\hline PD3. & Purposely fail to follow instructions & -0.03 & 0.06 & 0.01 & 0.06 & 0.82 \\
\hline PD4. & Give obscure instructions or information to others & 0.07 & -0.02 & 0.00 & 0.10 & 0.64 \\
\hline
\end{tabular}


Table 25. Inter-Item Correlations for Utilitarian Expectancies of Behavioral Engagement

\begin{tabular}{|c|c|c|c|c|c|c|c|c|c|c|}
\hline & Mean & $\mathrm{SD}$ & UEBE1 & UEBE2 & UEBE3 & UEBE4 & UEBE5 & UEBE6 & UEBE7 & UEBE8 \\
\hline UEBE1 & 4.60 & 1.52 & & & & & & & & \\
\hline UEBE2 & 4.34 & 1.46 & $.73^{* *}$ & & & & & & & \\
\hline UEBE3 & 4.22 & 1.55 & $.67^{* *}$ & $.83^{* *}$ & & & & & & \\
\hline UEBE4 & 4.88 & 1.40 & $.61^{* *}$ & $.69^{* *}$ & $.71^{* *}$ & & & & & \\
\hline UEBE5 & 5.56 & 1.27 & $.45^{* *}$ & $.44^{* * *}$ & $.40^{* *}$ & $.58^{* *}$ & & & & \\
\hline UEBE6 & 4.57 & 1.37 & $.39^{* *}$ & $.46^{* *}$ & $.51^{* *}$ & $.53^{* *}$ & $.44^{* *}$ & & & \\
\hline UEBE7 & 5.24 & 1.24 & $.43^{* *}$ & $.45^{* *}$ & $.45^{\text {** }}$ & $.57^{* *}$ & $.60^{* *}$ & $.57^{* *}$ & & \\
\hline UEBE8 & 5.41 & 1.25 & $.33^{* *}$ & $.36^{* *}$ & $.37^{* *}$ & $.52^{* *}$ & $.61^{* * *}$ & $.52^{* *}$ & $.75^{* *}$ & \\
\hline
\end{tabular}


Table 26. Exploratory Factor Analysis for Utilitarian Expectancies of Behavioral Engagement

\begin{tabular}{|c|c|c|}
\hline Item & Factor 1 & Factor 2 \\
\hline $\begin{array}{l}\text { UEBE1. I believe that working extra hour to solve } \\
\text { organizational problems will bring good outcomes to } \\
\text { me }\end{array}$ & 0.74 & 0.07 \\
\hline $\begin{array}{l}\text { UEBE2. I believe that working extra hour to meet additional } \\
\text { demand of the job will yield good result for me }\end{array}$ & 0.93 & -0.01 \\
\hline $\begin{array}{l}\text { UEBE3. I believe that doing extra works which beyond my } \\
\text { responsibility will be good for me }\end{array}$ & 0.90 & 0.00 \\
\hline $\begin{array}{l}\text { UEBE4. I believe that taking extra responsibility to deal will } \\
\text { new organizational challenges will be beneficial for me }\end{array}$ & 0.57 & 0.35 \\
\hline $\begin{array}{l}\text { UEBE5. I believe that proactively seeking a better way of doing } \\
\text { the job will yield good result for me }\end{array}$ & 0.10 & 0.66 \\
\hline $\begin{array}{l}\text { UEBE6. I believe that taking my own risk in initiating new ways } \\
\text { of doing things will be good for me }\end{array}$ & 0.25 & 0.50 \\
\hline $\begin{array}{l}\text { UEBE7. I believe that supporting new ideas that I believe will } \\
\text { bring comparative advantages to the organization will } \\
\text { be beneficial for me } \\
\text { UEBE8. I believe that actively monitoring the work environment } \\
\text { to respond quickly to changes will bring good } \\
\text { outcomes to me }\end{array}$ & 0.01 & 0.86 \\
\hline
\end{tabular}

Note. UEBE = Utilitarian expectancies of behavioral engagement. $\mathrm{N}=256$. Geomin rotation was used. The correlation between factors was .56. 
Table 27. Inter-Item Correlations for Normative Expectancies of Behavioral Engagement

\begin{tabular}{|c|c|c|c|c|c|c|c|c|c|c|}
\hline & Mean & SD & NEBE1 & NEBE2 & NEBE3 & NEBE4 & NEBE5 & NEBE6 & NEBE7 & NEBE8 \\
\hline NEBE1 & 4.77 & 1.45 & & & & & & & & \\
\hline NEBE2 & 4.49 & 1.48 & $.81^{* *}$ & & & & & & & \\
\hline NEBE3 & 4.40 & 1.51 & $.70^{* *}$ & $.82^{* *}$ & & & & & & \\
\hline NEBE4 & 4.87 & 1.40 & $.70^{* *}$ & $.63^{* *}$ & $.60^{* *}$ & & & & & \\
\hline NEBE5 & 5.31 & 1.18 & $.50^{* *}$ & $.42^{* *}$ & $.39^{* *}$ & $.65^{* *}$ & & & & \\
\hline NEBE6 & 4.80 & 1.29 & $.41^{* *}$ & $.47^{* *}$ & $.48^{* *}$ & $.56^{* *}$ & $.62^{* *}$ & & & \\
\hline NEBE7 & 5.28 & 1.15 & $.50^{* *}$ & $.43^{* *}$ & $.38^{* *}$ & $.57^{* *}$ & $.83^{* *}$ & $.60^{* *}$ & & \\
\hline NEBE8 & 5.22 & 1.18 & $.48^{* *}$ & $.42^{* *}$ & $.43^{* *}$ & $.58^{* *}$ & $.84^{* *}$ & $.63^{* *}$ & $.81^{* *}$ & \\
\hline
\end{tabular}


Table 28. Exploratory Factor Analysis for Normative Expectancies of Behavioral Engagement

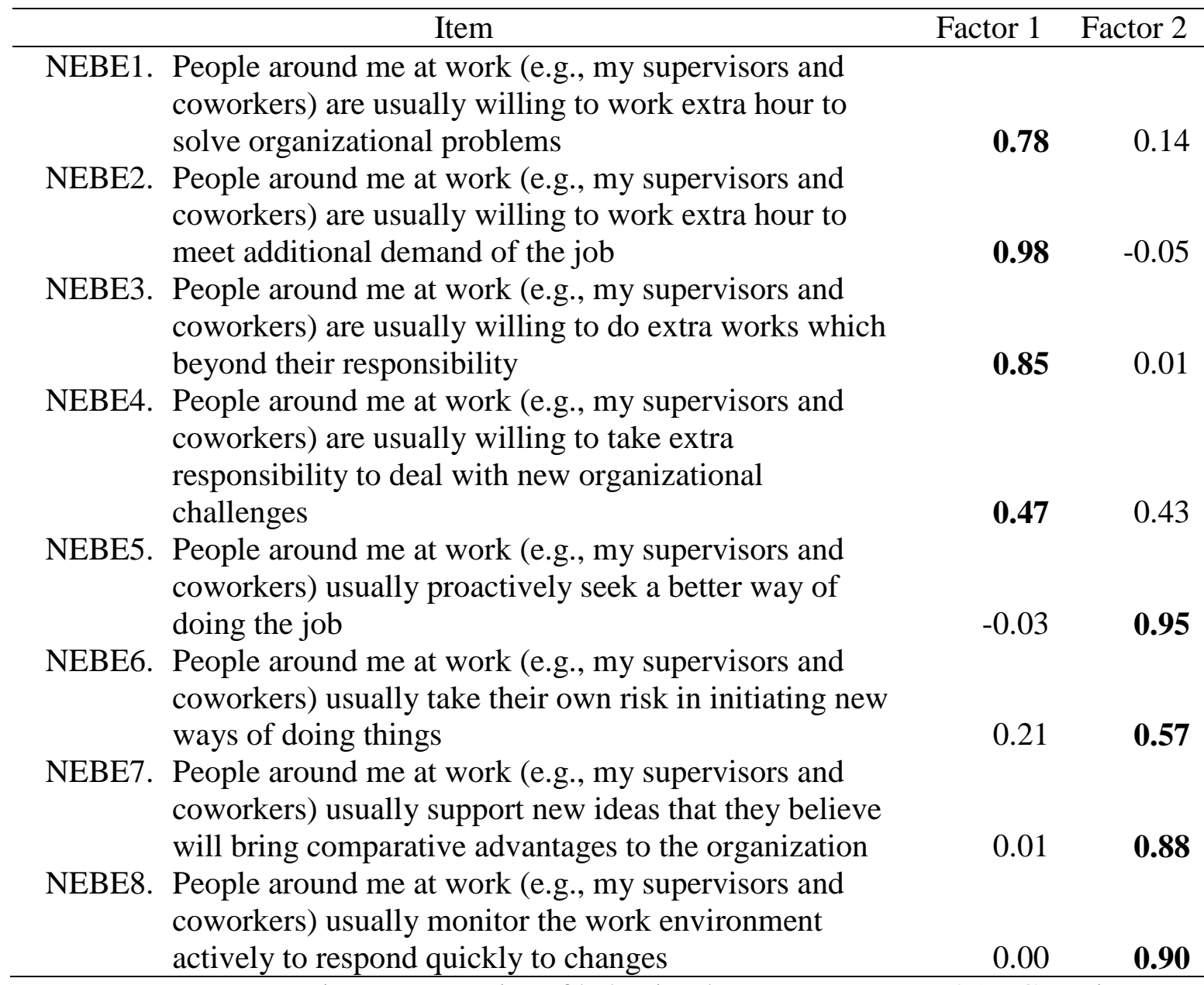

Note. NEBE = Normative expectancies of behavioral engagement. $\mathrm{N}=257$. Geomin rotation was used. The correlation between factors was .52 . 
Table 29. Inter-Item Correlations for Self-Identity Expectancies of Behavioral Engagement

\begin{tabular}{|c|c|c|c|c|c|c|c|c|c|c|}
\hline & Mean & SD & SEBE1 & SEBE2 & SEBE3 & SEBE4 & SEBE5 & SEBE6 & SEBE7 & SEBE8 \\
\hline SEBE1 & 5.36 & 1.25 & & & & & & & & \\
\hline SEBE2 & 5.12 & 1.35 & $.75^{* *}$ & & & & & & & \\
\hline SEBE3 & 5.33 & 4.04 & $24^{* *}$ & $.28^{* *}$ & & & & & & \\
\hline SEBE4 & 5.39 & 1.24 & $.70^{* *}$ & $.64^{* *}$ & $.25^{* *}$ & & & & & \\
\hline SEBE5 & 5.62 & 1.06 & $.66^{* *}$ & $.55^{* *}$ & $21^{* *}$ & $.69^{* *}$ & & & & \\
\hline SEBE6 & 5.15 & 1.25 & $.49^{* *}$ & $.57^{* *}$ & $.23^{* *}$ & $.61^{* *}$ & $.56^{* *}$ & & & \\
\hline SEBE7 & 5.51 & 1.08 & $63^{* *}$ & $.57^{* *}$ & $16^{* *}$ & $.64^{* *}$ & $.76^{* *}$ & $.62^{* *}$ & & \\
\hline SEBE8 & 5.61 & 1.06 & $.61^{* *}$ & $.56^{* *}$ & $20^{* *}$ & $.59^{* *}$ & $.79^{* *}$ & $.65^{* *}$ & $.76^{* *}$ & \\
\hline
\end{tabular}


Table 30. Exploratory Factor Analysis for Self-Identity Expectancies of Behavioral Engagement

\begin{tabular}{|c|c|c|}
\hline & Item & Factor 1 \\
\hline SEBE1. & $\begin{array}{l}\text { Working extra hour to solve organizational problems makes me } \\
\text { feel as a good member of the organization }\end{array}$ & 0.79 \\
\hline SEBE2. & $\begin{array}{l}\text { Working extra hour to meet additional demand of the job makes } \\
\text { me feel as a good member of the organization }\end{array}$ & 0.73 \\
\hline SEBE3. & $\begin{array}{l}\text { Doing extra works which beyond one's responsibility makes me } \\
\text { feel as a good member of the organization }\end{array}$ & 0.27 \\
\hline SEBE4. & $\begin{array}{l}\text { Taking extra responsibility to deal with new organizational } \\
\text { challenges makes me feel as a good member of the organization }\end{array}$ & 0.79 \\
\hline SEBE5. & $\begin{array}{l}\text { Proactively seeking a better way of doing the job makes me feel as } \\
\text { a good member of the organization }\end{array}$ & 0.87 \\
\hline SEBE6. & $\begin{array}{l}\text { Taking your own risk in initiating new ways of doing things makes } \\
\text { me feel as a good member of the organization }\end{array}$ & 0.72 \\
\hline SEBE7. & $\begin{array}{l}\text { Supporting new ideas that you believe will bring comparative } \\
\text { advantages to the organization }\end{array}$ & 0.85 \\
\hline SEBE8. & $\begin{array}{l}\text { Actively monitoring the work environment to respond quickly to } \\
\text { changes makes me feel as a good member of the organization }\end{array}$ & 0.85 \\
\hline
\end{tabular}

Note. SEBE = Self-identity expectancies of behavioral engagement. $\mathrm{N}=254$. Geomin rotation was used. 
Table 31. Inter-Item Correlations for Attitude toward Behavioral Engagement

\begin{tabular}{|c|c|c|c|c|c|c|c|c|c|c|c|c|c|}
\hline & Mean & SD & BGBE1 & BGBE2 & BGBE3 & BGBE4 & BGBE5 & BGBE6 & BGBE7 & BGBE8 & HBBE1 & HBBE2 & HBBE3 \\
\hline BGBE1 & 5.07 & 1.33 & & & & & & & & & & & \\
\hline BGBE2 & 4.95 & 1.20 & $.57^{* *}$ & & & & & & & & & & \\
\hline BGBE3 & 5.17 & 1.21 & $.53^{* *}$ & $.67^{* *}$ & & & & & & & & & \\
\hline BGBE4 & 5.83 & 0.93 & $.49^{* *}$ & $.50^{* *}$ & $.64^{* *}$ & & & & & & & & \\
\hline BGBE5 & 5.86 & 0.92 & $.41^{* *}$ & $.47^{* *}$ & $.58^{* *}$ & $.81^{* *}$ & & & & & & & \\
\hline BGBE6 & 5.17 & 1.14 & $.41^{* *}$ & $.55^{* *}$ & $.61^{* *}$ & $.56^{* *}$ & $.59^{* *}$ & & & & & & \\
\hline BGBE7 & 5.77 & 0.95 & $.46^{* *}$ & $.46^{* *}$ & $.57^{* *}$ & $.68^{* *}$ & $.77^{* *}$ & $.65^{* *}$ & & & & & \\
\hline BGBE8 & 5.79 & 0.96 & $.48^{* *}$ & $.47^{* *}$ & $.55^{* *}$ & $.65^{* *}$ & $.74^{* *}$ & $.61^{* *}$ & $.75^{* *}$ & & & & \\
\hline HBBE1 & 5.09 & 1.30 & $.50^{* *}$ & $.49^{* *}$ & $.49^{* *}$ & $.37^{* *}$ & $.43^{* *}$ & $.40^{* *}$ & $.46^{* * *}$ & $.41^{* *}$ & & & \\
\hline HBBE2 & 5.08 & 1.25 & $.50^{* *}$ & $.63^{* *}$ & $.54^{* *}$ & $.29^{* *}$ & $.35^{* *}$ & $.44^{* *}$ & $.40^{* *}$ & $.41^{* *}$ & $.70^{* *}$ & & \\
\hline HBBE3 & 5.21 & 1.21 & $.46^{* *}$ & $.61^{* *}$ & $.72^{* *}$ & $.47^{* *}$ & $.53^{* *}$ & $.60^{* *}$ & $.51^{* *}$ & $.53^{* *}$ & $.60^{* *}$ & $.66^{* *}$ & \\
\hline HBBE4 & 5.78 & 0.92 & $.51^{* *}$ & $.43^{* *}$ & $.52^{* *}$ & $.62^{* *}$ & $.66^{* *}$ & $.52^{* *}$ & $.65^{* *}$ & $.62^{* *}$ & $.52^{* *}$ & $.48^{* *}$ & $.59^{* *}$ \\
\hline HBBE5 & 5.84 & 0.92 & $.48^{* *}$ & $.41^{* *}$ & $.50^{* *}$ & $.62^{* *}$ & $.68^{* *}$ & $.51^{* *}$ & $.65^{* *}$ & $.64^{* *}$ & $.48^{* *}$ & $.41^{* *}$ & $.60^{* *}$ \\
\hline HBBE6 & 5.33 & 1.16 & $.42^{* *}$ & $.50^{* *}$ & $.52^{* *}$ & $.42^{* *}$ & $.41^{* *}$ & $.73^{* *}$ & $.47^{* *}$ & $.51^{* *}$ & $.35^{* *}$ & $.47^{* *}$ & $.63^{* *}$ \\
\hline HBBE7 & 5.81 & 0.87 & $.43^{* *}$ & $.44^{* *}$ & $.47^{* *}$ & $.55^{* *}$ & $.60^{* *}$ & $.51^{* *}$ & $.65^{* *}$ & $.56^{* *}$ & $.47^{* *}$ & $.40^{* *}$ & $.59^{* *}$ \\
\hline HBBE8 & 5.84 & 0.92 & $.40^{* *}$ & $.43^{* *}$ & $.51^{* *}$ & $.57^{* *}$ & $.63^{* *}$ & $.54^{* *}$ & $.63^{* *}$ & $.71^{* *}$ & $.37^{* *}$ & $.35^{* *}$ & $.56^{* *}$ \\
\hline
\end{tabular}

Note. BGBE = Attitude toward behavioral engagement measured by bad-good scale. HBBE = Attitude toward behavioral engagement

measured by harmful-beneficial scale. $\mathrm{N}=258$. $* * p<.01$ 
Table 31. Inter-Item Correlations for Attitude toward Behavioral Engagement (Continued)

\begin{tabular}{cccccccc}
\hline & Mean & SD & HBBE4 & HBBE5 & HBBE6 & HBBE7 & HBBE8 \\
\hline HBBE4 & 5.78 & 0.92 & & & & & \\
HBBE5 & 5.84 & 0.92 & $.77^{* *}$ & & & & \\
HBBE6 & 5.33 & 1.16 & $.51^{* *}$ & $.57^{* *}$ & & & \\
HBBE7 & 5.81 & 0.87 & $.67^{* * *}$ & $.73^{* *}$ & $.56^{* * *}$ & & \\
HBBE8 & 5.84 & 0.92 & $.70^{* *}$ & $.72^{* *}$ & $.55^{* *}$ & $.73^{* *}$ & \\
\hline
\end{tabular}

Note. $\mathrm{HBBE}=$ Attitude toward behavioral engagement measured by harmful-beneficial scale. $\mathrm{N}=258 . * * p<.01$. 
Table 32. Exploratory Factor Analysis for Attitude toward Behavioral Engagement

\begin{tabular}{llr}
\hline \multicolumn{1}{c}{ Item } & Factor 1 & Factor 2 \\
\hline BGBE1. Willing to work extra hour to solve organizational & & \\
problems/ & 0.21 & $\mathbf{0 . 4 9}$ \\
BGBE2. Willing to work extra hour to meet additional demand & & \\
of the job & 0.06 & $\mathbf{0 . 7 1}$ \\
BGBE3. Willing to do ex tra works which beyond one's & & \\
responsibility & 0.30 & $\mathbf{0 . 5 5}$ \\
BGBE4. Willing to take extra responsibility to deal with new & & \\
organizational challenges & $\mathbf{0 . 8 7}$ & -0.08 \\
BGBE5. Proactively seek a better way of doing the job & $\mathbf{0 . 9 5}$ & -0.11 \\
BGBE6. Take your own risk in initiating new ways of doing \\
things
\end{tabular}

Note. BGBE = Attitude toward behavioral engagement measured by bad-good scale.

$\mathrm{HBBE}=$ Attitude toward behavioral engagement measured by harmful-beneficial scale . $\mathrm{N}=258$. Geomin rotation was used. The correlation between the factors was .69 . 
Table 33. Inter-Item Correlations for Behavioral Engagement

\begin{tabular}{|c|c|c|c|c|c|c|c|c|c|c|}
\hline & Mean & SD & BE1 & BE2 & BE3 & BE4 & BE5 & BE6 & BE7 & BE8 \\
\hline BE1 & 5.72 & 1.08 & & & & & & & & \\
\hline BE2 & 5.67 & 1.12 & $.82^{* *}$ & & & & & & & \\
\hline BE3 & 5.66 & 1.03 & $.76^{* *}$ & $.82^{* *}$ & & & & & & \\
\hline BE4 & 5.72 & 0.90 & $.56^{* *}$ & $.58^{* *}$ & $.65^{* *}$ & & & & & \\
\hline BE5 & 5.70 & 0.95 & $.54^{* *}$ & $.53^{* *}$ & $.59^{* *}$ & $.85^{* *}$ & & & & \\
\hline BE6 & 5.42 & 1.09 & $.61^{* *}$ & $.57^{* *}$ & $.60^{* *}$ & $.68^{* *}$ & $.69^{* * *}$ & & & \\
\hline BE7 & 5.61 & 1.00 & $.55^{* *}$ & $.47^{* *}$ & $.53^{* *}$ & $.65^{* *}$ & $65^{* *}$ & $.74^{* *}$ & & \\
\hline BE8 & 5.61 & 0.94 & $.42^{* *}$ & $.45^{* *}$ & $.50^{* *}$ & $.66^{* *}$ & $.68^{* *}$ & $.69^{* *}$ & $.69^{* *}$ & \\
\hline
\end{tabular}

Note. $\mathrm{BE}=$ Behavioral engagement. $\mathrm{N}=257 .{ }^{*} * p<.01$. 
Organizational Commitment 163

Table 34. Exploratory Factor Analysis for Behavioral Engagement

\begin{tabular}{lrr}
\hline \multicolumn{1}{c}{ Item } & Factor 1 & Factor 2 \\
\hline BE1. Willing to work extra hour to solve organizational problems & $\mathbf{0 . 8 6}$ & 0.02 \\
BE2. Willing to work extra hour to meet additional demand of & & \\
the job & $\mathbf{1 . 0 0}$ & -0.08 \\
BE3. Willing to do extra works which beyond one's & & \\
responsibility & $\mathbf{0 . 7 8}$ & 0.13 \\
BE4. Willing to take extra responsibility to deal with new & 0.08 & $\mathbf{0 . 8 3}$ \\
$\quad \begin{array}{l}\text { organizational challenges } \\
\text { BE5. Proactively seek a better way of doing the job }\end{array}$ & -0.02 & $\mathbf{0 . 9 1}$ \\
BE6. Take your own risk in initiating new ways of doing things & 0.16 & $\mathbf{0 . 7 0}$ \\
BE7. Support new ideas that you believe will bring comparative & & \\
$\quad \begin{array}{l}\text { advantages to the organization } \\
\text { BE8. Actively monitor the work environment to respond quickly } \\
\text { to changes }\end{array}$ & 0.04 & $\mathbf{0 . 7 5}$ \\
\hline
\end{tabular}

Note. $\mathrm{BE}=$ Behavioral engagement. $\mathrm{N}=257$. Geomin rotation was used. The correlation between the factors was .69 . 
Table 35. Confirmatory Factor Analysis Results for Scales involved Behavioral Engagement

\begin{tabular}{|c|c|c|c|c|c|c|c|c|}
\hline Model & $\mathrm{N}$ & $\mathrm{X}^{2}$ & df & CFI & RMSEA & SRMR & $\Delta \mathrm{X}^{2}$ & $\Delta \mathrm{df}$ \\
\hline \multicolumn{9}{|l|}{ Utilitarian expectancies of behavioral engagement } \\
\hline One factor model (without item 1-4) & 256 & 1.78 & 2 & 1.00 & 0.000 & 0.01 & & \\
\hline \multicolumn{9}{|l|}{ Normative expectancies of behavioral engagement } \\
\hline One factor model (without item 1-4) & 255 & 0.55 & 2 & 1.00 & 0.000 & 0.004 & & \\
\hline \multicolumn{9}{|l|}{ Self-identity expectancies of behavioral engagement } \\
\hline One factor model & 254 & 161.64 & 20 & 0.89 & 0.167 & 0.05 & & \\
\hline One factor model (without item 3) & 254 & 152.92 & 14 & 0.90 & 0.198 & 0.05 & $8.72^{n s}$ & 6 \\
\hline One factor model (without item 3 and with correlated error terms) & 254 & 90.48 & 13 & 0.94 & 1.53 & 0.04 & $62.44 * *$ & 1 \\
\hline \multicolumn{9}{|l|}{ Attitude toward behavioral engagement } \\
\hline One factor model (without item 1-3) & 258 & 216.568 & 33 & 0.92 & 0.147 & 0.04 & & \\
\hline \multicolumn{9}{|l|}{ Behavioral engagement } \\
\hline One factor model (without item 1-3) & 257 & 74.17 & 5 & 0.93 & 0.232 & 0.04 & & \\
\hline
\end{tabular}

Note. ${ }^{* *} p<.01 . n s=$ non-significant 
Table 36. Exploratory Factor Analysis for the Final Scales involved Behavioral Engagement

\begin{tabular}{|c|c|c|c|c|c|c|}
\hline & Item & $\begin{array}{c}\text { Factor } \\
1\end{array}$ & $\begin{array}{l}\text { Factor } \\
2\end{array}$ & $\begin{array}{l}\text { Factor } \\
3\end{array}$ & $\begin{array}{c}\text { Factor } \\
4\end{array}$ & $\begin{array}{c}\text { Factor } \\
5\end{array}$ \\
\hline UEBE5. & I believe that proactively seeking a better way of doing the job will yield good result for me & 0.54 & 0.12 & 0.16 & 0.00 & -0.05 \\
\hline UEBE6. & I believe that taking my own risk in initiating new ways of doing things will be good for me & 0.72 & -0.13 & -0.01 & 0.03 & 0.04 \\
\hline UEBE7. & $\begin{array}{l}\text { I believe that supporting new ideas that I believe will bring comparative advantages to the } \\
\text { organization will be beneficial for me }\end{array}$ & 0.84 & 0.10 & 0.00 & -0.02 & -0.03 \\
\hline UEBE8. & $\begin{array}{l}\text { I believe that actively monitoring the work environment to respond quickly to changes will } \\
\text { bring good outcomes to me }\end{array}$ & 0.76 & 0.01 & 0.14 & 0.01 & 0.02 \\
\hline NEBE5. & $\begin{array}{l}\text { People around me at work (e.g., my supervisors and coworkers) usually proactively seek a } \\
\text { better way of doing the job }\end{array}$ & 0.03 & 0.90 & 0.04 & -0.04 & 0.03 \\
\hline NEBE6. & $\begin{array}{l}\text { People around me at work (e.g., my supervisors and coworkers) usually take their own risk } \\
\text { in initiating new ways of doing things }\end{array}$ & 0.16 & 0.64 & -0.08 & 0.02 & 0.01 \\
\hline NEBE7. & $\begin{array}{l}\text { People around me at work (e.g., my supervisors and coworkers) usually support new ideas } \\
\text { that they believe will bring comparative advantages to the organization }\end{array}$ & -0.04 & 0.87 & 0.06 & 0.04 & -0.02 \\
\hline NEBE8. & $\begin{array}{l}\text { People around me at work (e.g., my supervisors and coworkers) usually monitor the work } \\
\text { environment actively to respond quickly to changes }\end{array}$ & 0.01 & 0.89 & 0.00 & 0.02 & 0.01 \\
\hline SEBE5. & $\begin{array}{l}\text { Proactively seeking a better way of doing the job makes me feel as a good member of the } \\
\text { organization }\end{array}$ & -0.02 & 0.01 & 0.88 & -0.02 & 0.04 \\
\hline SEBE6. & $\begin{array}{l}\text { Taking your own risk in initiating new ways of doing things makes me feel as a good } \\
\text { member of the organization }\end{array}$ & 0.16 & -0.10 & 0.67 & 0.01 & -0.04 \\
\hline SEBE7. & Supporting new ideas that you believe will bring comparative advantages to the organization & 0.01 & 0.10 & 0.78 & 0.02 & 0.04 \\
\hline SEBE8. & $\begin{array}{l}\text { Actively monitoring the work environment to respond quickly to changes makes me feel as a } \\
\text { good member of the organization }\end{array}$ & 0.01 & 0.04 & 0.88 & 0.02 & -0.01 \\
\hline HBBE5. & Proactively seek a better way of doing the job & 0.00 & 0.01 & 0.06 & 0.83 & 0.00 \\
\hline HBBE6. & Take your own risk in initiating new ways of doing things & 0.16 & -0.01 & -0.09 & 0.62 & 0.09 \\
\hline HBBE7. & Support new ideas that you believe will bring comparative advantages to the organization & -0.08 & 0.03 & 0.00 & 0.90 & -0.10 \\
\hline HBBE8. & Actively monitor the work environment to respond quickly to changes & 0.05 & -0.01 & 0.04 & 0.82 & 0.04 \\
\hline BE5. & Proactively seek a better way of doing the job & -0.01 & 0.05 & 0.01 & -0.01 & 0.79 \\
\hline BE6. & Take your own risk in initiating new ways of doing things & 0.02 & 0.00 & -0.01 & -0.01 & 0.87 \\
\hline BE7. & Support new ideas that you believe will bring comparative advantages to the organization & -0.08 & -0.02 & 0.05 & -0.02 & 0.85 \\
\hline BE8. & Actively monitor the work environment to respond quickly to changes & 0.04 & 0.00 & -0.03 & 0.07 & 0.80 \\
\hline
\end{tabular}


Table 37. Confirmatory Factor Analysis Results for the Hypothesized Models

\begin{tabular}{|c|c|c|c|c|c|c|c|}
\hline Model & $\mathrm{X}^{2}$ & df & CFI & RMSEA & SRMR & $\Delta X^{2}$ & $\Delta \mathrm{df}$ \\
\hline \multicolumn{8}{|l|}{ Considerate voice } \\
\hline Overall Model with all organizational commitment items & 1844.24 & 874 & 0.87 & 0.006 & 0.085 & & \\
\hline Overall Model with modified organizational items & 1049.96 & 566 & 0.93 & 0.058 & 0.053 & $794.28 * *$ & 308 \\
\hline \multicolumn{8}{|l|}{ Production deviance } \\
\hline Overall Model with all organizational commitment items & 1506.70 & 674 & 0.89 & 0.069 & 0.085 & & \\
\hline Overall Model with modified organizational items & 719.52 & 406 & 0.96 & 0.055 & 0.051 & $787.19 * *$ & 268 \\
\hline \multicolumn{8}{|l|}{ Behavioral engagement } \\
\hline Overall Model with all organizational commitment items & 1560.29 & 674 & 0.84 & 0.071 & 0.09 & & \\
\hline Overall Model with modified organizational items & 792.83 & 406 & 0.92 & 0.061 & 0.055 & $767.46 * *$ & 268 \\
\hline
\end{tabular}

Note. $\mathrm{N}=258 . * * p<.01$ 
Table 38. Exploratory Factor Analysis for Organizational Commitment Scale

\begin{tabular}{|c|c|c|c|c|}
\hline Item & Factor 1 & Factor 2 & Factor 3 & Factor 4 \\
\hline AC1. I would be very happy to spend the rest of my career at this organization. & 0.09 & -0.07 & 0.64 & 0.08 \\
\hline AC2. I really feel as if this organization's problems are my own. & 0.08 & -0.07 & 0.54 & 0.15 \\
\hline AC3. I do not feel like "part of the family" at my organization. & 0.79 & -0.05 & 0.03 & -0.08 \\
\hline AC4. I do not feel "emotionally attached" to this organization. & 0.91 & 0.03 & -0.03 & -0.02 \\
\hline AC5. This organization has a great deal of personal meaning for me. & 0.14 & 0.13 & 0.70 & -0.24 \\
\hline AC6. I do not feel a strong sense of belonging to my organization. & 0.27 & -0.24 & 0.28 & -0.04 \\
\hline CC1. It would be very hard for me to leave my organization right now, even if I wanted to. & 0.08 & 0.49 & 0.08 & 0.11 \\
\hline $\begin{array}{l}\text { CC2. Too much of my life would be disrupted if I decided I wanted to leave my } \\
\text { organization right now. }\end{array}$ & 0.11 & 0.55 & 0.15 & 0.09 \\
\hline CC3. Right now, staying with my organization is a matter of necessity as much as desire. & 0.24 & 0.49 & 0.36 & 0.04 \\
\hline CC4. I believe that I have too few options to consider leaving this organization. & -0.01 & 0.68 & -0.03 & 0.19 \\
\hline $\begin{array}{l}\text { CC5. One of the few negative consequences of leaving this organization would be the } \\
\text { scarcity of available alternatives. }\end{array}$ & -0.13 & 0.69 & -0.02 & -0.03 \\
\hline $\begin{array}{l}\text { CC6. One of the major reasons I continue to work for this organization is that leaving } \\
\text { would require considerable personal sacrifice; another organization may not match } \\
\text { the overall benefits I have here. }\end{array}$ & -0.11 & 0.75 & 0.00 & -0.20 \\
\hline $\begin{array}{l}\text { CC7. If I had not already put so much of myself into this organization, I might consider } \\
\text { working elsewhere. }\end{array}$ & -0.21 & 0.40 & -0.11 & -0.04 \\
\hline NC1. I do not feel any obligation to remain with my current employer. & 0.26 & -0.25 & 0.00 & 0.23 \\
\hline $\begin{array}{l}\text { NC2. Even if it were to my advantage, I do not feel it would be right to leave my } \\
\text { organization now. }\end{array}$ & -0.01 & 0.08 & 0.49 & 0.36 \\
\hline NC3. I would feel guilty if I left my organization now. & -0.12 & 0.08 & 0.34 & 0.56 \\
\hline NC4. This organization deserves my loyalty. & -0.04 & -0.10 & 0.88 & -0.02 \\
\hline $\begin{array}{l}\text { NC5. I would not leave my organization right now because I have a sense of obligation to } \\
\text { the people in it. }\end{array}$ & -0.04 & -0.01 & 0.75 & 0.09 \\
\hline NC6. I owe a great deal to my organization. & -0.03 & 0.00 & 0.89 & -0.31 \\
\hline
\end{tabular}


Table 39. Exploratory Factor Analysis for Affective Commitment, Continuance Commitment, and Normative Commitment

\begin{tabular}{|c|c|c|c|c|c|c|}
\hline \multirow[t]{2}{*}{ Item } & \multicolumn{2}{|c|}{$\begin{array}{l}\text { Affective } \\
\text { commitment }\end{array}$} & \multicolumn{2}{|c|}{$\begin{array}{l}\text { Continuance } \\
\text { commitment }\end{array}$} & \multicolumn{2}{|c|}{$\begin{array}{l}\text { Normative } \\
\text { commitment }\end{array}$} \\
\hline & Factor 1 & Factor 2 & Factor 1 & Factor 2 & Factor 1 & Factor 2 \\
\hline AC1. I would be very happy to spend the rest of my career at this organization. & 0.79 & 0.00 & & & & \\
\hline AC2. I really feel as if this organization's problems are my own. & 0.69 & -0.02 & & & & \\
\hline AC3. I do not feel like "part of the family" at my organization. & -0.02 & 0.88 & & & & \\
\hline AC4. I do not feel "emotionally attached" to this organization. & 0.08 & 0.82 & & & & \\
\hline AC5. This organization has a great deal of personal meaning for me. & 0.57 & 0.14 & & & & \\
\hline AC6. I do not feel a strong sense of belonging to my organization. & 0.24 & 0.34 & & & & \\
\hline $\begin{array}{l}\text { CC1. It would be very hard for me to leave my organization right now, even if I } \\
\text { wanted to. }\end{array}$ & & & 0.64 & 0.01 & & \\
\hline $\begin{array}{l}\text { CC2. Too much of my life would be disrupted if I decided I wanted to leave my } \\
\text { organization right now. }\end{array}$ & & & 0.73 & 0.01 & & \\
\hline $\begin{array}{l}\text { CC3. Right now, staying with my organization is a matter of necessity as much as } \\
\text { desire. }\end{array}$ & & & 0.71 & -0.08 & & \\
\hline CC4. I believe that I have too few options to consider leaving this organization. & & & 0.28 & 0.54 & & \\
\hline $\begin{array}{l}\text { CC5. One of the few negative consequences of leaving this organization would be } \\
\text { the scarcity of available alternatives. }\end{array}$ & & & 0.00 & 0.78 & & \\
\hline $\begin{array}{l}\text { CC6. One of the major reasons I continue to work for this organization is that leaving } \\
\text { would require considerable personal sacrifice; another organization may not } \\
\text { match the overall benefits I have here. }\end{array}$ & & & 0.02 & 0.74 & & \\
\hline $\begin{array}{l}\text { CC7. If I had not already put so much of myself into this organization, I might } \\
\text { consider working elsewhere. }\end{array}$ & & & -0.22 & 0.63 & & \\
\hline NC7. I do not feel any obligation to remain with my current employer. & & & & & 0.07 & 0.06 \\
\hline $\begin{array}{l}\text { NC8. Even if it were to my advantage, I do not feel it would be right to leave my } \\
\text { organization now. }\end{array}$ & & & & & 0.23 & 0.61 \\
\hline NC9. I would feel guilty if I left my organization now. & & & & & 0.00 & 0.74 \\
\hline NC10. This organization deserves my loyalty. & & & & & 0.83 & 0.04 \\
\hline $\begin{array}{l}\text { NC11. I would not leave my organization right now because I have a sense of } \\
\text { obligation to the people in it. }\end{array}$ & & & & & 0.68 & 0.17 \\
\hline NC12. I owe a great deal to my organization. & & & & & 0.94 & -0.23 \\
\hline
\end{tabular}


Table 40. Means, Standard Deviations, and Correlations among Study Variables

\begin{tabular}{|c|c|c|c|c|c|c|c|c|c|c|c|c|c|c|}
\hline & Mean & SD & Age & Gender & Edu & Tenure & $\mathrm{AC}$ & $\mathrm{CC}$ & $\mathrm{NC}$ & UECV & NECV & SECV & ATTCV & $\mathrm{CV}$ \\
\hline Age & 27.10 & 2.44 & & & & & & & & & & & & \\
\hline Gender & 0.27 & 0.45 & -0.13 & & & & & & & & & & & \\
\hline Edu & 15.41 & 1.50 & -0.13 & $.21 * *$ & & & & & & & & & & \\
\hline Tenure & 3.37 & 2.24 & $.80 * *$ & $-.25 * *$ & $-.29 * *$ & & & & & & & & & \\
\hline $\mathrm{AC}$ & 5.17 & 1.12 & 0.08 & -0.07 & 0.01 & 0.05 & $(0.74)$ & & & & & & & \\
\hline $\mathrm{CC}$ & 4.53 & 1.13 & 0.08 & -0.03 & 0.06 & -0.04 & $.38 * *$ & $(0.72)$ & & & & & & \\
\hline $\mathrm{NC}$ & 4.80 & 1.01 & 0.01 & -0.01 & 0.02 & -0.04 & $.69 * *$ & $.38 * *$ & $(0.82)$ & & & & & \\
\hline UECV & 5.62 & 0.97 & 0.01 & 0.07 & 0.09 & -0.01 & $.53 * *$ & $.24 * *$ & $.46^{* *}$ & $(0.91)$ & & & & \\
\hline NECV & 5.53 & 1.03 & -0.02 & 0.03 & 0.02 & -0.04 & $.51 * *$ & $.24 * *$ & $.44 * *$ & $.71 * *$ & $(0.94)$ & & & \\
\hline SECV & 5.49 & 0.91 & -0.06 & 0.05 & 0.02 & -0.02 & $.45 * *$ & $.25 * *$ & $.43 * *$ & $.68 * *$ & $.74 * *$ & $(0.93)$ & & \\
\hline ATTCV & 5.87 & 0.75 & 0.03 & $.15^{*}$ & -0.03 & 0.02 & $.25^{* *}$ & 0.07 & $.17 * *$ & $.35 * *$ & $.34 * *$ & $.37 * *$ & $(0.90)$ & \\
\hline $\mathrm{CV}$ & 5.92 & 0.91 & 0.09 & -0.07 & -0.03 & 0.04 & 0.12 & 0.05 & 0.11 & 0.11 & 0.09 & 0.12 & 0.12 & $(0.93)$ \\
\hline UEPD & 2.12 & 1.17 & -0.10 & 0.04 & 0.01 & -0.12 & $-.45 * *$ & $-.14 *$ & $-.26 * *$ & $-.40 * *$ & $-.35 * *$ & $-.34 * *$ & $-.20 * *$ & -0.02 \\
\hline NEPD & 2.51 & 1.41 & -0.04 & 0.00 & 0.02 & 0.04 & $-.45 * *$ & $-.13 *$ & $-.33 * *$ & $-.45 * *$ & $-.47 * *$ & $-.46 * *$ & $-.19 * *$ & $-.17 * *$ \\
\hline SEPD & 3.96 & 2.03 & -0.07 & 0.06 & 0.05 & 0.02 & 0.00 & 0.07 & 0.02 & $.19 * *$ & 0.11 & $.16^{*}$ & $.15^{*}$ & 0.07 \\
\hline ATTPD & 2.05 & 1.30 & 0.03 & -0.03 & 0.04 & 0.01 & -0.12 & 0.01 & -0.11 & $-.22 * *$ & $-.14^{*}$ & $-.23 * *$ & $-.34 * *$ & $-.19 * *$ \\
\hline PD & 1.10 & 0.26 & -0.03 & $.16^{* *}$ & 0.07 & -0.09 & 0.01 & 0.02 & 0.01 & -0.04 & -0.01 & -0.05 & 0.04 & $-.34 * *$ \\
\hline UEBE & 5.19 & 1.06 & 0.01 & 0.12 & 0.05 & -0.02 & $.27 * *$ & $.20 * *$ & $.22 * *$ & $.50^{* *}$ & $.49 * *$ & $.57 * *$ & $.30 * *$ & 0.02 \\
\hline NEBE & 5.15 & 1.06 & -0.12 & 0.02 & 0.01 & -0.11 & $.42 * *$ & $.20 * *$ & $.43^{* *}$ & $.61 * *$ & $.69 * *$ & $.65^{* *}$ & $.28 * *$ & 0.05 \\
\hline SEBE & 5.47 & 0.97 & -0.06 & 0.06 & 0.03 & -0.02 & $.41 * *$ & $.23 * *$ & $.32 * *$ & $.55^{* *}$ & $.58 * *$ & $.66^{* *}$ & $.30 * *$ & 0.05 \\
\hline АТTBE & 5.71 & 0.82 & -0.03 & $.16^{*}$ & -0.03 & -0.07 & $.24 * *$ & $.16^{* *}$ & $.21 * *$ & $.31 * *$ & $.32 * *$ & $.34 * *$ & $.74 * *$ & $.17 * *$ \\
\hline $\mathrm{BE}$ & 5.59 & 0.87 & -0.02 & -0.03 & -0.05 & -0.04 & 0.12 & 0.10 & 0.08 & $.15^{*}$ & 0.09 & 0.12 & $.16^{*}$ & $.51 * *$ \\
\hline
\end{tabular}

Note. $\mathrm{Edu}=$ Education. Tenure $=$ Organizational tenure. $\mathrm{AC}=$ Affective commitment. $\mathrm{CC}=$ Continuance commitment. NC $=$ Normative commitment. $\mathrm{UECV}=$ Utilitarian expectancies of considerate voice. NECV = Normative expectancies of considerate voice. SECV $=$ Self-identity expectancies of considerate voice. $\mathrm{ATTCV}=$ Attitude toward considerate voice. $\mathrm{CV}=$ Considerate voice. $\mathrm{UEPD}=\mathrm{Utilitarian}$ expectancies of production deviance. $\mathrm{NEPD}=$ Normative expectancies of production deviance. SEPD $=$ Self-identity expectancies of production deviance. ATTPD $=$ Attitude toward production. PD $=$ Production deviance. UEBE $=$ Utilitarian expectancies of behavioral engagement. NEBE $=$ Normative expectancies of behavioral engagement. SEBE $=$ Self-identity expectancies of behavioral engagement. ATTBE = Attitude toward behavioral engagement. BE = Behavioral engagement. Values on the diagonal in the parenthesis reflect the Cronbach's alpha for the measure. $\mathrm{N}=258$. $* p<.05 * * p<.01$ 
Table 40. Means, Standard Deviations, and Correlations among Study Variables (Continued)

\begin{tabular}{|c|c|c|c|c|c|c|c|c|c|c|c|c|}
\hline & Mean & SD & UEPD & NEPD & SEPD & ATTPD & PD & UEBE & NEBE & SEBE & ATTBE & $\mathrm{BE}$ \\
\hline UEPD & 2.12 & 1.17 & $(0.89)$ & & & & & & & & & \\
\hline NEPD & 2.51 & 1.41 & $.52 * *$ & $(0.96)$ & & & & & & & & \\
\hline SEPD & 3.96 & 2.03 & -0.04 & 0.01 & (0.99) & & & & & & & \\
\hline ATTPD & 2.05 & 1.30 & 0.12 & 0.10 & $-.15^{*}$ & $(0.97)$ & & & & & & \\
\hline PD & 1.10 & 0.26 & 0.12 & 0.07 & -0.03 & $.22 * *$ & $(0.72)$ & & & & & \\
\hline UEBE & 5.19 & 1.06 & $-.24 * *$ & $-.34 * *$ & $.14 *$ & $-.17 * *$ & 0.02 & $(0.85)$ & & & & \\
\hline NEBE & 5.15 & 1.06 & $-.21 * *$ & $-.41 * *$ & 0.02 & -0.12 & -0.07 & $.49 * *$ & $(0.91)$ & & & \\
\hline SEBE & 5.47 & 0.97 & $-.30 * *$ & $-.31 * *$ & $.18^{* *}$ & $-.18 * *$ & -0.02 & $.53 * *$ & $.55^{* *}$ & $(0.90)$ & & \\
\hline ATTBE & 5.71 & 0.82 & $-.17 * *$ & $-.18 * *$ & $.20 * *$ & $-.29 * *$ & 0.01 & $.35 * *$ & $.31 * *$ & $.31 * *$ & (0.87) & \\
\hline $\mathrm{BE}$ & 5.59 & 0.87 & -0.08 & -0.08 & 0.02 & -0.08 & $-.33 * *$ & 0.02 & $.13 *$ & 0.09 & $.23 * *$ & $(0.90)$ \\
\hline
\end{tabular}

Note. UEPD = Utilitarian expectancies of production deviance. NEPD = Normative expectancies of production deviance. SEPD = Self-identity

expectancies of production deviance. ATTPD $=$ Attitude toward production. $\mathrm{PD}=$ Production deviance. UEBE $=$ Utilitarian expectancies of behavioral engagement. NEBE $=$ Normative expectancies of behavioral engagement. SEBE $=$ Self-identity expectancies of behavioral engagement. ATTBE $=$ Attitude toward behavioral engagement. $\mathrm{BE}=$ Behavioral engagement. Values on the diagonal in the parenthesis reflect the Cronbach's alpha for the measure. $\mathrm{N}=$ 258. $* p<.05 * * p<.01$ 
Table 41. Standardized Structural Path Coefficients for Study Variables on Mediator and Considerate Voice in the Full Mediation Model (M1)

\begin{tabular}{|c|c|c|c|}
\hline & & $\begin{array}{l}\text { Attitude toward } \\
\text { considerate voice }\end{array}$ & Considerate voice $(\mathrm{CV})$ \\
\hline \multirow[t]{4}{*}{ Step 1} & Gender & $0.16^{* *}$ & -0.07 \\
\hline & Continuance commitment & 0.02 & 0.02 \\
\hline & Normative commitment & $0.25 * *$ & 0.10 \\
\hline & Attitude toward considerate voice & & $0.12 \dagger$ \\
\hline \multirow[t]{5}{*}{ Step 2} & Gender & $0.14 *$ & -0.08 \\
\hline & Continuance commitment & $-0.68 \dagger$ & 0.02 \\
\hline & Normative commitment & $-2.44 \dagger$ & 0.10 \\
\hline & Attitude toward considerate voice & & $0.13 \dagger$ \\
\hline & Affective commitment (AC) & $3.11 *$ & \\
\hline \multirow[t]{8}{*}{ Step 3} & Gender & $0.13 *$ & -0.08 \\
\hline & Continuance commitment & -0.17 & 0.02 \\
\hline & Normative commitment & -0.56 & 0.10 \\
\hline & Attitude toward considerate voice & & $0.13 \dagger$ \\
\hline & Affective commitment & 0.68 & \\
\hline & Utilitarian expectancies of considerate voice (UECV) & 0.12 & \\
\hline & Normative expectancies of considerate voice (NECV) & 0.00 & \\
\hline & Self-identity expectancies of considerate voice (SECV) & $0.29 \dagger$ & \\
\hline \multirow[t]{4}{*}{ Step 4} & Indirect effects from $\mathrm{AC}$ to $\mathrm{CV}$ & & 0.09 \\
\hline & Indirect effects from UECV to $\mathrm{CV}$ & & 0.02 \\
\hline & Indirect effects from NECV to $\mathrm{CV}$ & & 0.00 \\
\hline & Indirect effects from SECV to CV & & 0.04 \\
\hline \multicolumn{4}{|c|}{ Note. $\mathrm{N}=255 . \dagger=p<.10 . * p<.05 . * * p<.01$} \\
\hline
\end{tabular}


Table 42. Standardized Structural Path Coefficients for Study Variables on Mediator and Production Deviance in the Full Mediation Model (M1)

\begin{tabular}{|c|c|c|c|}
\hline & & $\begin{array}{c}\text { Attitude toward } \\
\text { production deviance }\end{array}$ & Production deviance (PD) \\
\hline \multirow[t]{4}{*}{ Step 1} & Gender & -0.01 & $0.15^{*}$ \\
\hline & Continuance commitment & 0.09 & 0.05 \\
\hline & Normative commitment & $-0.16 \dagger$ & 0.00 \\
\hline & Attitude toward production deviance & & $0.31 * *$ \\
\hline \multirow[t]{5}{*}{ Step 2} & Gender & -0.02 & $0.15^{*}$ \\
\hline & Continuance commitment & 0.20 & 0.05 \\
\hline & Normative commitment & 0.28 & 0.00 \\
\hline & Attitude toward production deviance & & $0.31 * *$ \\
\hline & Affective commitment (AC) & -0.52 & \\
\hline \multirow[t]{8}{*}{ Step 3} & Gender & -0.01 & $0.15^{*}$ \\
\hline & Continuance commitment & 0.24 & 0.05 \\
\hline & Normative commitment & 0.35 & 0.00 \\
\hline & Attitude toward production deviance & & $0.31 * *$ \\
\hline & Affective commitment & -0.62 & \\
\hline & Utilitarian expectancies of production deviance (UEPD) & -0.02 & \\
\hline & Normative expectancies of production deviance (NEPD) & -0.01 & \\
\hline & Self-identity expectancies of production deviance (SEPD) & $-0.21 * *$ & \\
\hline \multirow[t]{4}{*}{ Step 4} & Indirect effects from $\mathrm{AC}$ to $\mathrm{PD}$ & & -0.19 \\
\hline & Indirect effects from UEPD to PD & & -0.01 \\
\hline & Indirect effects from NEPD to PD & & 0.00 \\
\hline & Indirect effects from SEPD to PD & & $-0.06 *$ \\
\hline \multicolumn{4}{|c|}{ Note. $\mathrm{N}=255 . \dagger=p<.10 . * p<.05 . * * p<.01$} \\
\hline
\end{tabular}


Table 43. Standardized Structural Path Coefficients for Study Variables on Mediator and Behavioral Engagement in the Full Mediation Model (M1)

\begin{tabular}{|c|c|c|c|}
\hline & & $\begin{array}{c}\text { Attitude toward } \\
\text { behavioral engagement }\end{array}$ & $\begin{array}{c}\text { Behavioral engagement } \\
\text { (BE) }\end{array}$ \\
\hline \multirow[t]{4}{*}{ Step 1} & Gender & $0.17 * *$ & -0.06 \\
\hline & Continuance commitment & 0.10 & 0.07 \\
\hline & Normative commitment & $0.22 *$ & 0.01 \\
\hline & Attitude toward behavioral engagement & & $0.25 * *$ \\
\hline \multirow[t]{5}{*}{ Step 2} & Gender & $0.13 *$ & -0.06 \\
\hline & Continuance commitment & -0.49 & 0.07 \\
\hline & Normative commitment & $-1.96 \dagger$ & 0.02 \\
\hline & Attitude toward behavioral engagement & & $0.25^{* *}$ \\
\hline & Affective commitment (AC) & $2.54 *$ & \\
\hline \multirow[t]{8}{*}{ Step 3} & Gender & $0.13 *$ & -0.06 \\
\hline & Continuance commitment & -0.01 & 0.07 \\
\hline & Normative commitment & -0.13 & 0.01 \\
\hline & Attitude toward behavioral engagement & & $0.24 * *$ \\
\hline & Affective commitment & 0.24 & \\
\hline & Utilitarian expectancies of behavioral engagement (UEBE) & $0.21 *$ & \\
\hline & Normative expectancies of behavioral engagement (NEBE) & 0.11 & \\
\hline & Self-identity expectancies of behavioral engagement (SEBE) & 0.09 & \\
\hline \multirow[t]{4}{*}{ Step 4} & Indirect effects from $\mathrm{AC}$ to $\mathrm{BE}$ & & 0.06 \\
\hline & Indirect effects from UEBE to $\mathrm{BE}$ & & $0.05 \dagger$ \\
\hline & Indirect effects from NEBE to $\mathrm{BE}$ & & 0.03 \\
\hline & Indirect effects from SEBE to BE & & 0.02 \\
\hline \multicolumn{4}{|c|}{ Note. $\mathrm{N}=255 . \dagger=p<.10 . * p<.05 . * * p<.01$} \\
\hline
\end{tabular}


Organizational Commitment 174

Table 44. Bootstrap Confidence Intervals for the Indirect Effects

\begin{tabular}{ccc}
\hline \multicolumn{1}{c}{ Predictor } & Mediators & $\begin{array}{c}95 \% \text { Confidence } \\
\text { Interval }\end{array}$ \\
\hline Considerate voice & Attitude toward considerate voice & {$[-0.286,0.436]$} \\
Affective commitment & {$[-0.075,0.109]$} \\
Utilitarian expectancies & & {$[-0.066,0.066]$} \\
Normative expectancies & & {$[-0.063,0.138]$} \\
Self-identity expectancies & & {$[-0.164,0.105]$} \\
Production deviance & Attitude toward production deviance & {$[-0.032,0.030]$} \\
Affective commitment & & {$[-0.011,0.010]$} \\
Utilitarian expectancies & & {$[-0.012,0.001]$} \\
Normative expectancies & & \\
Self-identity expectancies & & {$[-0.458,0.547]$} \\
Behavioral engagement & Attitude toward behavioral engagement & {$[-0.033,0.116]$} \\
Affective commitment & & {$[-0.041,0.077]$} \\
Utilitarian expectancies & & {$[-0.101,0.135]$} \\
Normative expectancies & &
\end{tabular}


Figure 1. Proposed Full Mediation Model $\left(\mathrm{M}_{1}\right)$

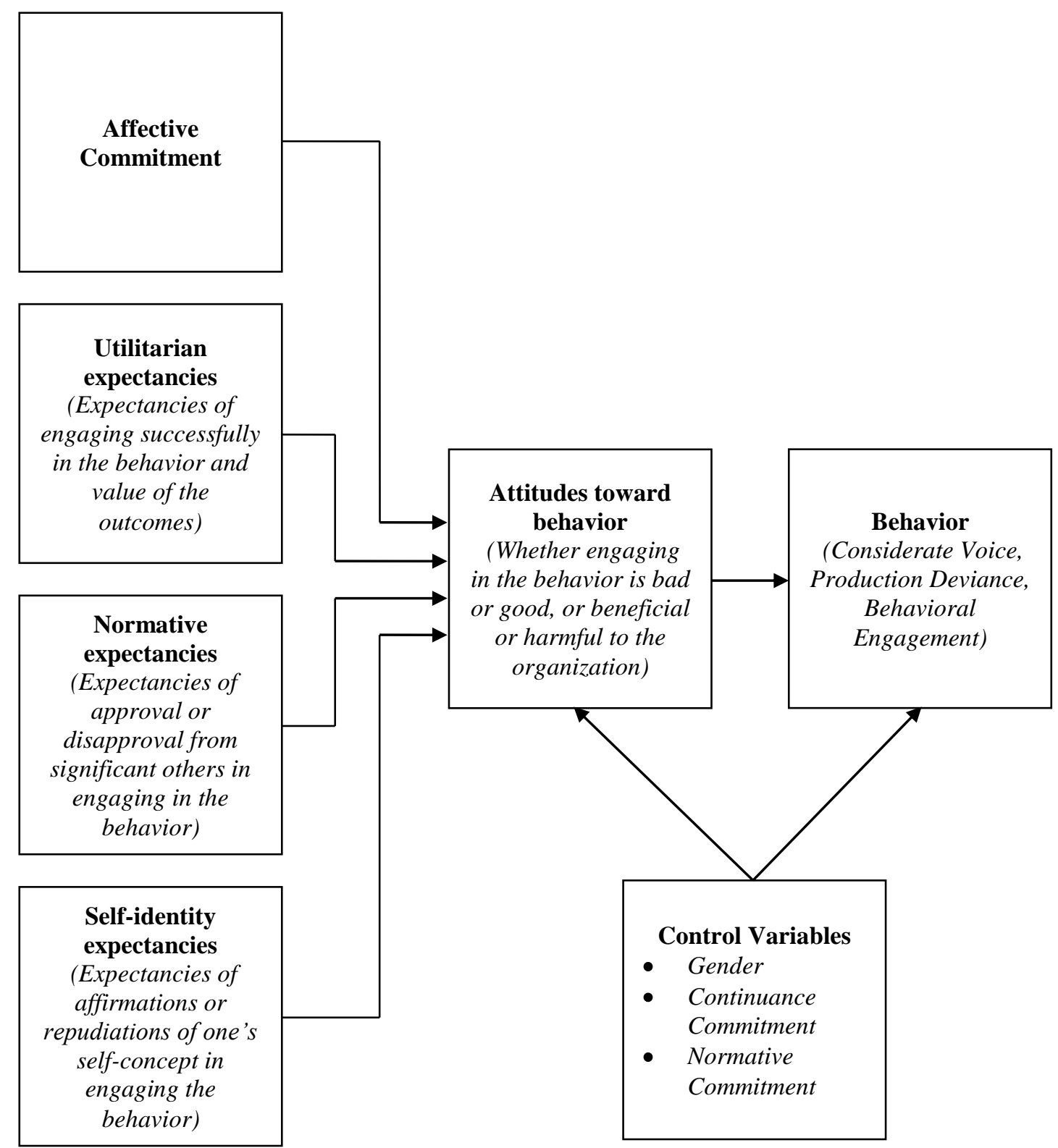


Figure 2. Partial Mediation Model $\left(\mathrm{M}_{2}\right)$

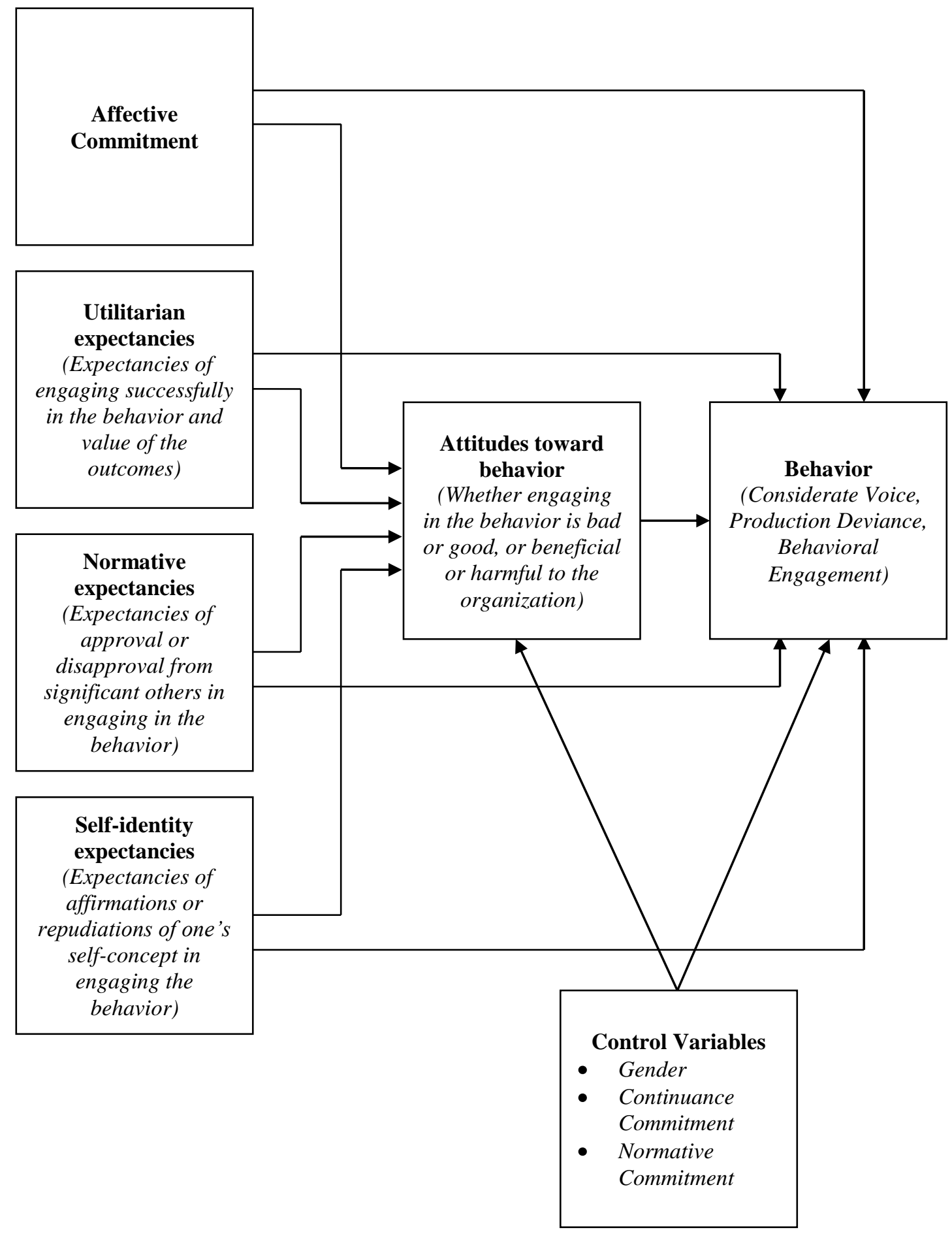


Figure 3. Non-Mediation Model $\left(\mathrm{M}_{3}\right)$

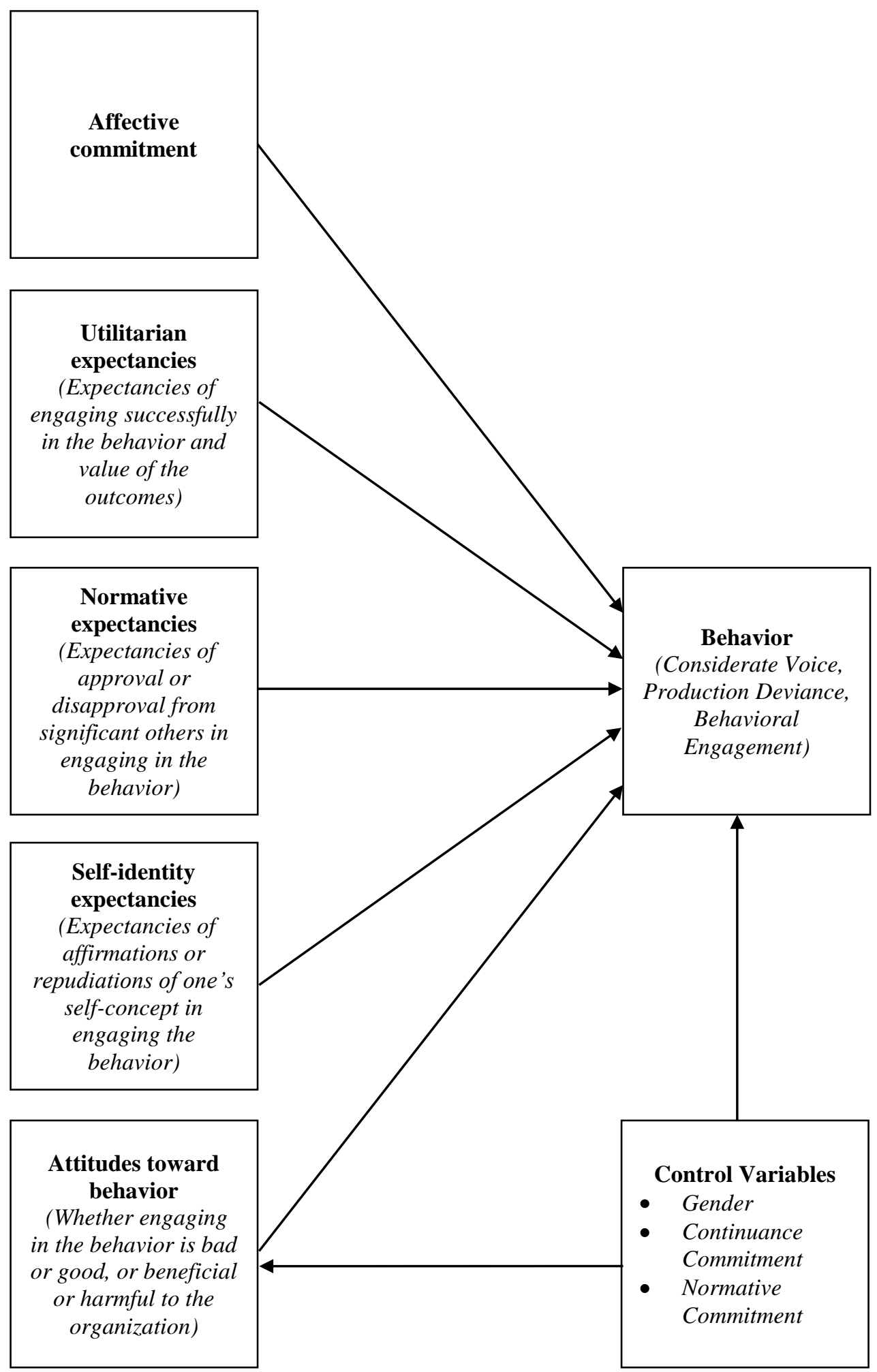


Figure 4. Full Mediation Model $\left(\mathrm{M}_{1}\right)$ for Considerate Voice $\dagger=p<.10$.

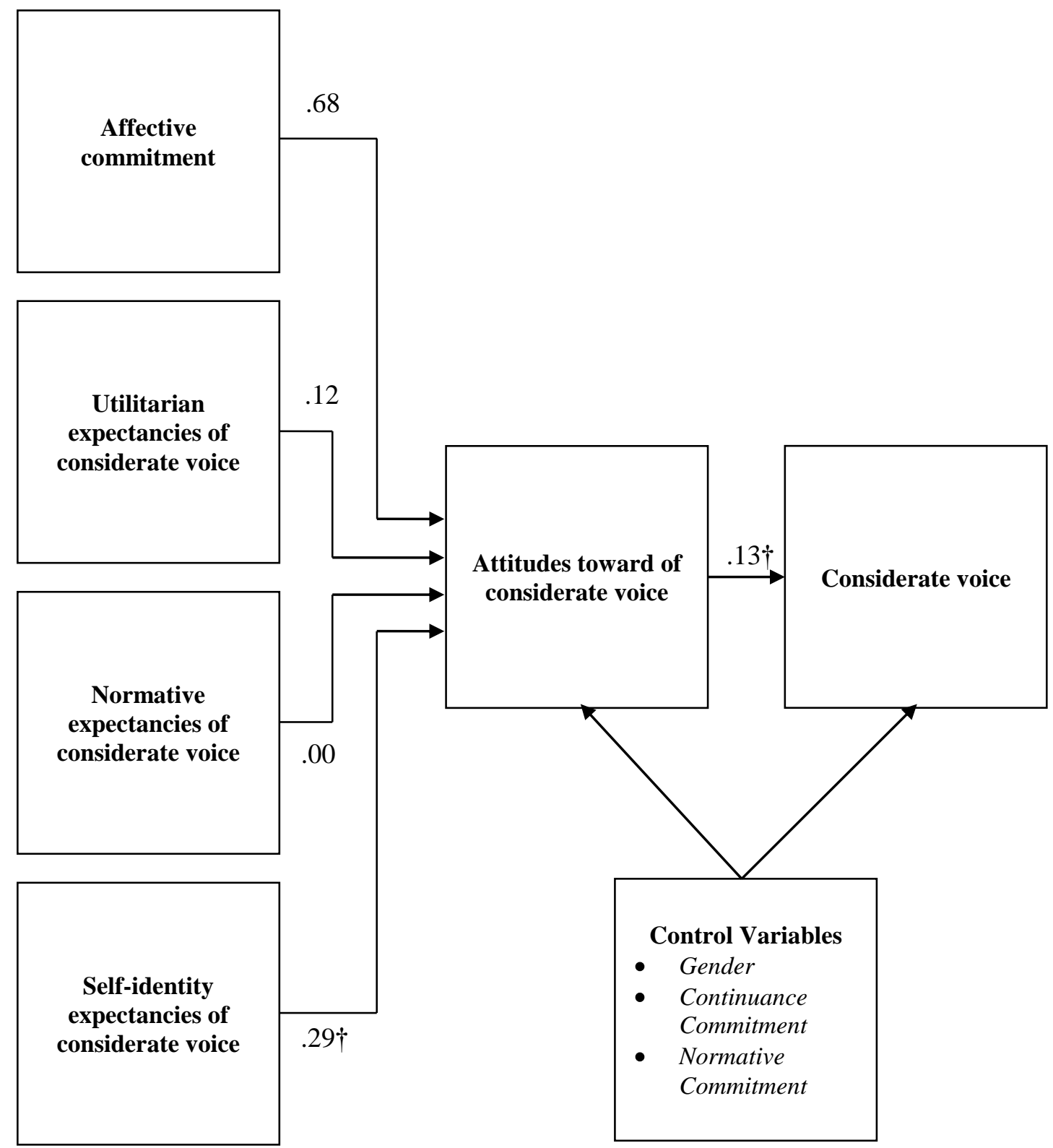


Figure 5. Full Mediation Model $\left(\mathrm{M}_{1}\right)$ for Production Deviance

$* * p<.01$

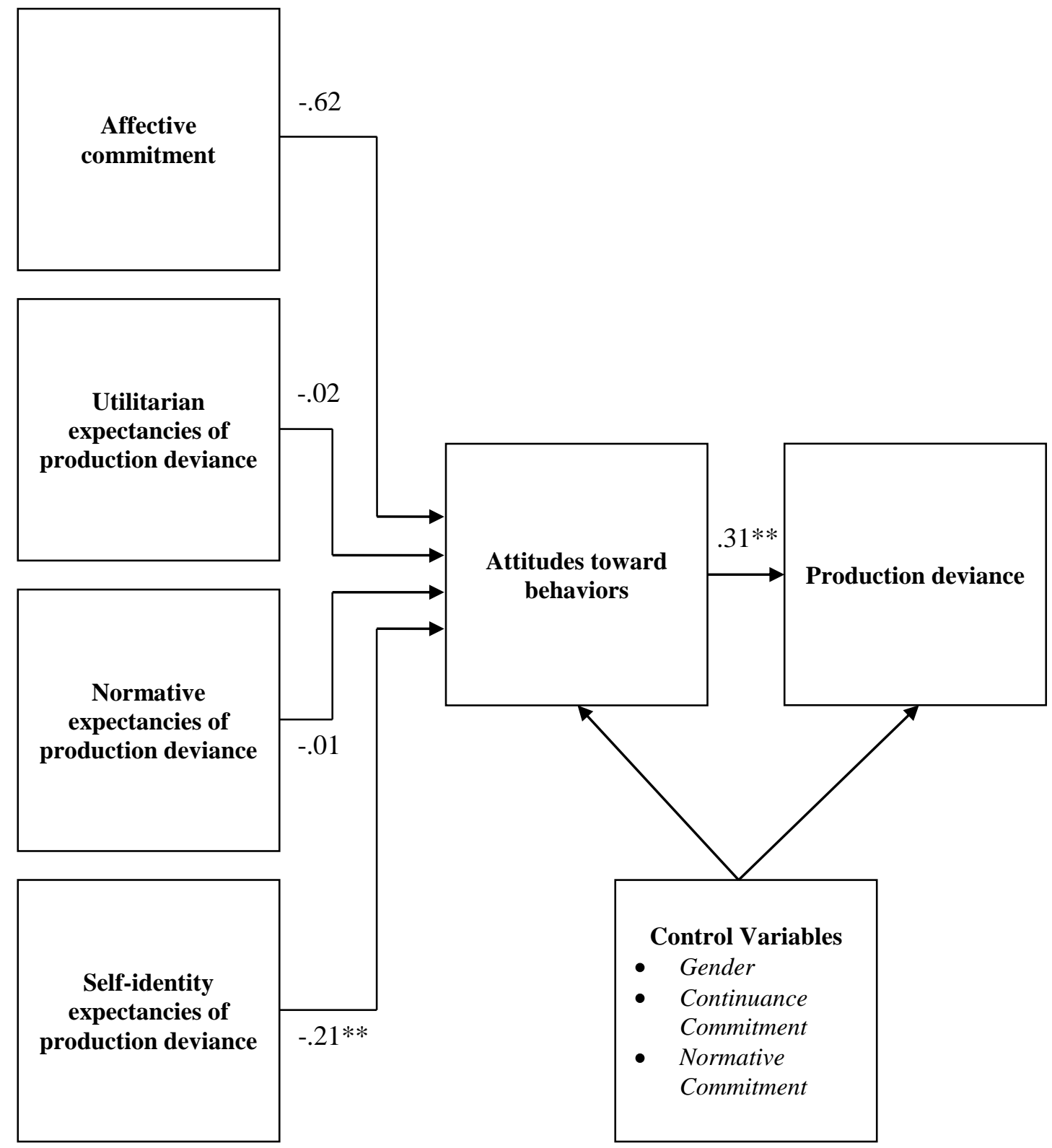


Figure 6. Full Mediation Model $\left(\mathrm{M}_{1}\right)$ for Behavior Engagement

$* p<.05 . * * p<.01$

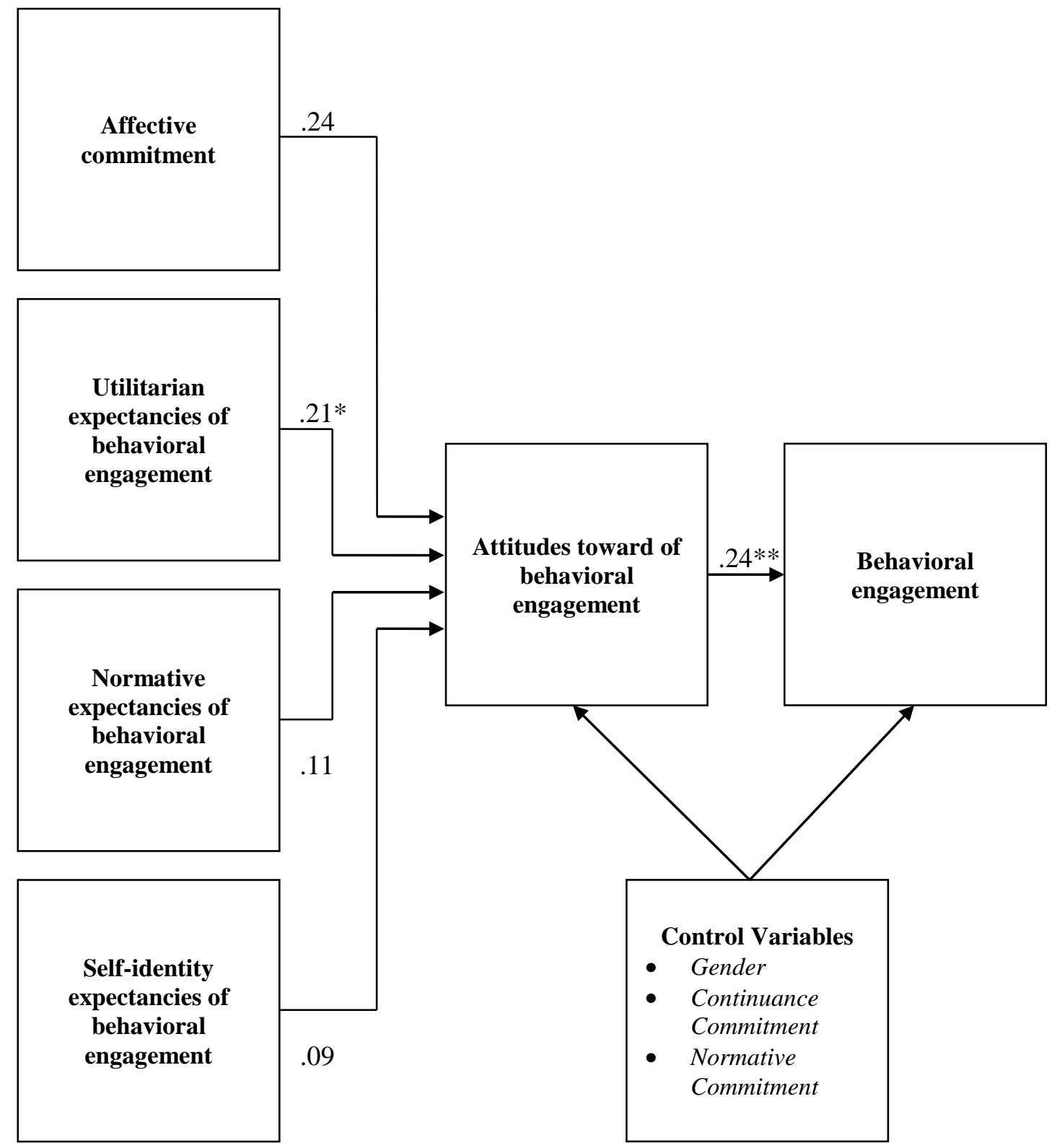




\section{References}

Ajzen, I. (1985). From intentions to actions: A theory of planned behavior. In J. Kuhl \& J. Beckmann (Eds.), Action-control: From cognition to behavior (pp. 11-39). Heidelberg: Springer.

Ajzen, I. (1988). Attitudes, personality, and behavior. Milton Keynes, UK: Open University Press.

Ajzen, I. (1991). The theory of planned behavior. Organizational Behavior and Human Decision Processes, 50, 179-211.

Ajzen, I. (2002). Constructing a TPB questionnaire: Conceptual and methodological considerations. Retrieved December 4, 2011, from http://socgeo.ruhosting.nl/html/files/spatbeh/tpb.measurement.pdf

Ajzen, I., \& Fishbein, M. (1977). Attitude-behavior relations: A theoretical analysis and review of empirical research. Psychological Bulletin, 84, 888-918.

Ajzen, I., \& Fishbein, M. (1980). Understanding attitudes and predicting social behavior. Englewood Cliffs, N.J.: Prentice-Hall.

Ajzen, I., \& Fishbein, M. (2005). The influence of attitudes on behavior. In D. Albarracín, B. T. Johnson, \& M. P. Zanna (Eds.), The handbook of attitudes (pp. 173-221). Mahwah N.J.: Lawrence Erlhaum Associates.

Ajzen, I., Brown, T. C., \& Carvajal, F. (2004). Explaining the discrepancy between intentions and actions: the case of hypothetical bias in contingent valuation. Personality and Social Psychology Bulletin, 30, 1108-1121. 
Ajzen, I., Timko, C., \& White, J. B. (1982). Self-monitoring and the attitude-behavior relation. Journal of Personality and Social Psychology, 42, 426-435.

Allen, D. G., Shore, Lynn M., \& Griffeth, R. W. (2003). The role of perceived organizational support and supportive human resource practices in the turnover process. Journal of Management, 29(1), 99-118.

Allen, N. J., \& Meyer, J. P. (1990). The measurement and antecedents of affective, continuance and normative commitment to the organization. Journal of Occupational Psychology, 63(1), 1-18

Allen, N. J., \& Meyer, J. P. (1996). Affective, continuance, and normative commitment to the organization: An examination of construct validity. Journal of Vocational Behavior, 49, 252-276.

Allport, G. W. (1935). Attitudes. In C. Murchison (Ed.), Handbook of Social Psychology. Worchester, MA: Clark University Press.

Alutto, J. A., Hrebiniak, L. G., \& Alonso, R. C. (1973). On operationalizing the concept of commitment. Social Forces, 51, 448-454. University of North Carolina Press.

Anderson, J. C., \& Gerbing, D. W. (1988). Structural equation modeling in practice: A review and recommended two-step approach. Psychological Bulletin, 103, 411423.

Atkinson, J. W. (1964). Introduction to motivation. Princeton, NJ: Van Nostrand.

Bandura, A. (1977). Self-efficacy: Toward a unifying theory of behavioral change. Psychological Review, 84, 191-215. 
Bandura, A. (1982). The assessment and predictive generality of self-percepts of efficacy. Journal of Behavior Therapy and Experimental Psychiatry, 13, 195-199.

Beck, K., \& Wilson, C. (2000). Development of affective organizational commitment: A cross-sequential examination of change with tenure. Journal of Vocational Behavior, 56(1), 114-136.

Becker, H. S. (1960). Notes on the concept of commitment. The American Journal of Sociology, 66(1), 32-40.

Begley, T. M., \& Czajka, J. M. (1993). Panel analysis of the moderating effects of commitment on job satisfaction, intent to quit, and health following organizational change. Journal of Applied Psychology, 78, 552-556.

Bentein, K., Vandenberg, R., Vandenberghe, C., \& Stinglhamber, F. (2005). The role of change in the relationship between commitment and turnover: A latent growth modeling approach. Journal of Applied Psychology, 90, 468-482.

Blau, P. M. (1964). Exchange and power in social life. New York: Wiley.

Bozeman, D. P., \& Perrewe, P. L. (2001). The effect of item content overlap on organizational commitment questionnaire-turnover cognitions relationships. Journal of Applied Psychology, 86(1), 161-173.

Brayfield, A. H., \& Crockett, W. H. (1955). Employee attitudes and employee performance. Psychological Bulletin, 52, 396-424.

Brockner, J., Ackerman, G., Greenberg, Jerald, Gelfand, M. J., Francesco, A. M., Chen, Z. X., Leung, K., et al. (2001). Culture and procedural justice: The influence of 
Organizational Commitment 184

power distance on reactions to voice. Journal of Experimental Social Psychology, $37,300-315$.

Buchanan, B. (1974). Building organizational commitment: The socialization of managers in work organizations. Administrative Science Quarterly, 19, 533-546.

Bunderson, J. S. (2001). How work ideologies shape the psychological contracts of professional employees: Doctors' responses to perceived breach. Journal of Organizational Behavior, 22, 717-741.

Cassar, V. (2001). Violating psychological contract terms amongst Maltese public service employees: occurrence and relationships. Journal of Managerial Psychology, 16, 194-208.

Cassar, V., \& Briner, R. B. (2010). The relationship between psychological contract breach and organizational commitment: Exchange imbalance as a moderator of the mediating role of violation. Journal of Vocational Behavior, 78, 283-289.

Charng, H.-W., Piliavin, J. A., \& Callero, P. L. (1988). Role identity and reasoned action in the prediction of repeated behavior. Social Psychology Quarterly, 51, 303-317.

Churchill, G. A. J. (1979). A paradigm for developing better measures of marketing constructs. Journal of Marketing Research, 16(1), 64-73.

Cocroft, B. A. K., \& Ting-Toomey, S. (1994). Facework in Japan and the United States. International Journal of Intercultural Relations, 18, 469-506.

Cohen, A. (2003). Multiple commitments in the workplace An integrative approach. Mahwah N.J.: Lawrence Erlhaum Associates. 
Cohen, A. (2007). Commitment before and after: An evaluation and reconceptualization of organizational commitment. Human Resource Management Review, 17, 336354.

Conway, J. M., \& Huffcutt, A. I. (1997). Psychometric properties of multisource performance ratings: A meta-analysis of subordinate, supervisor, peer, and selfratings. Human Performance, 10, 331-360.

Cooper-Hakim, A., \& Viswesvaran, C. (2005). The construct of work commitment: Testing an integrative framework. Psychological Bulletin, 131, 241-259.

Coyle-Shapiro, J., \& Kessler, I. (2000). Consequences of the psychological contract for the employment relationship: A large scale survey. Journal of Management Studies, 37, 903-930.

Crawford, T. J., \& Boyer, R. (1985). Salient consequences, cultural values, and childbearing intentions. Journal of Applied Social Psychology, 15(1), 16-30.

Cropanzano, R., James, K., \& Konovsky, M. A. (1993). Dispositional affectivity as a predictor of work attitudes and job performance. Journal of Organizational Behavior, 14, 595-606.

Dalal, R. S. (2005). A meta-analysis of the relationship between organizational citizenship behavior and counterproductive work behavior. Journal of Applied Psychology, 90, 1241-1255.

Davidson, A. R., \& Jaccard, J. J. (1975). Population psychology: A new look at an old problem. Journal of Personality and Social Psychology, 31, 1073-1082. 
Organizational Commitment 186

DeFleur, M. L., \& Westie, F. R. (1958). Verbal attitudes and overt acts: An experiment on the salience of attitudes. American Sociological Review, 23, 667-673. American Sociological Society.

Dean, L. R. (1958). Interaction, reported and observed: The case of one local union. Human Organization, 17, 36-44.

Eagly, A. H., \& Chaiken, S. (1993). The psychology of attitudes. Fort Worth, TX: Harcourt Brace Jovanovich College Publishers.

Eisenberger, R., Huntington, R., Hutchison, S., \& Sowa, D. (1986). Perceived organizational support. Journal of Applied Psychology, 71, 500-507.

Erdheim, J., Wang, M., \& Zickar, M. J. (2006). Linking the Big Five personality constructs to organizational commitment. Personality and Individual Differences, 41, 959-970.

Fabrigar, L. R., MacDonald, T. K., \& Wegener, D. T. (2005). The structure of attitudes. In D. Albarracín, B. T. Johnson, \& M. P. Zanna (Eds.), The handbook of attitudes (pp. 79-124). Mahwah N.J.: Lawrence Erlhaum Associates.

Farrell, D. (1983). Exit, voice, loyalty, and neglect as responses to job dissatisfaction: A multidimensional scaling study. Academy of Management Journal, 26, 596-607.

Fazio, R. H. (1986). How do attitudes guide behavior? In R. M. Sorrentino \& E. T. Higgins (Eds.), (pp. 204-243). New York: Guilford Press.

Fazio, R. H. (1990). Multiple processes by which attitudes guide behavior: The MODE model as an integrative framework. In M. P. Zanna (Ed.), (pp. 75-109). New York: Academic Press. 
Fazio, R. H., \& Towles-Schwen, T. (1999). The MODE model of attitude-behavior processes. In S. Chaiken \& Y. Trope (Eds.), (pp. 97-116). New York: Guilford.

Feather, N. T. (1982). Expectations and actions: Expectancy-value models in psychology. Hillsdale, NJ: Erlbaum.

Fine, S., Horowitz, I., Weigler, H., \& Basis, L. (2010). Is good character good enough? The effects of situational variables on the relationship between integrity and counterproductive work behaviors. Human Resource Management Review, 20(1), 73-84.

Fiorito, J., Bozeman, D. P., Young, A., \& Meurs, J. A. (2007). Organizational commitment, human resource practices, and organizational characteristics. Journal of Managerial Issues, 19, 186-207.

Fishbein, M., \& Ajzen, I. (1975). Belief, attitude, intention, and behavior : an introduction to theory and research. Reading, Mass.: Addison-Wesley.

Fishbein, M., \& Ajzen, I. (1974). Attitudes towards objects as predictors of single and multiple behavorial criteria. Psychological Review, 81(1). 59-74.

Fishbein, M., Middlestadt, S., \& Chung, J. (1986). Predicting participation and choice: First-time voters in U.S. partisan elections. In S. Kraus \& R. M. Perlott (Eds.), (pp. 65-82). Beverly Hills, CA: Sage.

Gelfand, M. J., Erez, M., \& Aycan, Z. (2007). Cross-cultural organizational behavior. Annual Review of Psychology, 58, 479-514.

Gelfand, M. J., Nishii, L. H., \& Raver, J. L. (2006). On the nature and importance of cultural tightness-looseness. The Journal of Applied Psychology, 91, 1225-1244. 
Organizational Commitment 188

Glasman, L. R., \& Albarracín, D. (2006). Forming attitudes that predict future behavior: A meta-analysis of the attitude-behavior relation. Psychological Bulletin, 132, 778-822.

Gong, Y., Law, K. S., Chang, S., \& Xin, K. R. (2009). Human resources management and firm performance: The differential role of managerial affective and continuance commitment. Journal of Applied Psychology, 94(1), 263-275.

Gouldner, A. W. (1960). The norm of reciprocity: A preliminary statement. American sociological review, 161-178.

Grant, A. M., Dutton, J. E., \& Rosso, B. D. (2008). Giving commitment: Employee support programs and the prosocial sensemaking process. Academy of Management Journal, 51, 898-918.

Greenberg, J., \& Baron, A. B. (2003). Behavior in organizations (8th ed.). Upper Saddle River, NJ: Prentice Hall.

Guzzo, R. A., Noonan, K. A., \& Elron, E. (1994). Expatriate managers and the psychological contract. Journal of Applied Psychology, 79, 617-626.

Hagedoorn, M., Yperen, N. W. van, Vliert, E. van de, \& Buunk, B. P. (1999). Employees' reactions to problematic events: A circumplex structure of five categories of responses, and the role of job satisfaction. Journal of Organizational Behavior, 20, 309-321.

Harrison, D. A., Newman, D. A., \& Roth, P. L. (2006). How important are job attitudes? Meta-analytic comparisons of integrative behavioral outcomes and time sequences. Academy of Management Journal, 49, 305-325. 
Hinkin, T. R. (1995). A review of scale development practices in the study of organizations. Journal of Management, 21, 967-988.

Hinkin, T. R. (1998). A brief tutorial on the development of measures for use in survey questionnaires. Organizational Research Methods, 1(1), 104-121.

Hirschman, A. O. (1970). Exit, voice, and loyalty; responses to decline in firms, organizations, and states. Cambridge, Mass.: Harvard University Press.

Hofmann, D., \& Morgeson, F. P. (1999). Safety-related behavior as a social exchange: The role of perceived organizational support and leader-member exchange. Journal of Applied Psychology, 84, 286-96.

Hofstede, G. H. (1980). Culture's consequences: International differences in workrelated values. Beverly Hills CA: SAGE Publications.

Hofstede, G. H. (2011). National culture - Geert Hofstede. Retrieved December 5, 2011, from http://geert-hofstede.com/website/index.php

Holt, J. L., \& DeVore, C. J. (2005). Culture, gender, organizational role, and styles of conflict resolution: A meta-analysis. International Journal of Intercultural Relations, 29, 165-196.

House, R. J., Shane, S. A., \& Herold, D. M. (1996). Rumors of the death of dispositional research are vastly exaggerated. The Academy of Management Review, 21(1), 203-224. Academy of Management.

Hu, L.-tze, \& Bentler, P. (1999). Cutoff criteria for fit indexes in covariance structure analysis: Conventional criteria versus new alternatives. Structural Equation Modeling: A Multidisciplinary Journal, 6(1), 1-55. Taylor \& Francis Group. 
Organizational Commitment 190

Hulin, C. L. (2002). Lessons from industrial and organizational psychology. In J. M. Herman \& F. Drasgow (Eds.), (pp. 3-22). Mahwah, NJ: Erlbaum.

Hung, D. K. M., Ansari, M. A., \& Aafaqi, R. (2004). Fairness of human resource management practices, leader-member exchange and organizational commitment. Asian Academy of Management Journal, 9(1), 99-120.

Iaffaldano, M. T., \& Muchinsky, P. M. (1985). Job satisfaction and job performance: A meta-analysis. Psychological Bulletin, 97, 251-273.

Iverson, R. D. (1999). An event history analysis of employee turnover: The case of hospital employees in Australia. Human Resource Management Review, 9, 397418.

Kenny, D. A., Kaniskan, B., \& McCoach, D. B. (2011). The performance of RMSEA in models with small degrees of freedom. Unpublished paper, University of Connecticut.

King, G. W. (1975). An analysis of attitudinal and normative variables as predictors of intentions and behavior. Speech Monographs, 42, 237-244.

Ko, J.-W., Price, J. L., \& Mueller, C. W. (1997). Assessment of Meyer and Allen's threecomponent model of organizational commitment in South Korea. Journal of Applied Psychology, 82, 961-973.

Kraft, P., Rise, J., Sutton, S., \& Røysamb, E. (2005). Perceived difficulty in the theory of planned behaviour: perceived behavioural control or affective attitude? British Journal of Social Psychology, 44, 479-496. 
Kwon, K., Bae, J., \& Lawler, J. J. (2010). High commitment HR practices and top performers. Management International Review, 50(1), 57-80. Gabler Verlag.

LaPiere, R. T. (1934). Attitudes vs. actions. Social Forces, 13, 230-237. The Williams and Wilkins Press.

Lam, S. S. K., Hui, C., \& Law, K. S. (1999). Organizational citizenship behavior: Comparing perspectives of supervisors and subordinates across four international samples. Journal of Applied Psychology, 84, 594-601.

Lawton, R., Conner, M., \& McEachan, R. (2005). Desire or reason: Predicting health behaviors from affective and cognitive attitudes. Health Psychology, 28(1), 56-65.

Lee, J. A., \& Yang, C. (2005). Factor structure of organizational commitment: Differences between U.S. and South Korean samples 1, 2, 3. Psychological Reports, 96, 595-602. Ammons Scientific, Ltd. P.O. Box 9229, Missoula, MT 59807-9229 USA.

Lee, K., Allen, N. J., Meyer, J. P., \& Rhee, K.-Y. (2001). The three-component model of organisational commitment: An application to South Korea. Applied Psychology: An International Review, 50, 596-614. doi:Article

Levinson, H. (1965). Reciprocation: The relationship between man and organization. Administrative Science Quarterly, 9, 370-390.

Liska, A. E. (1984). A critical examination of the causal structure of the Fishbein/Ajzen attitude-behavior model. Social Psychology Quarterly, 47(1), 61-74. American Sociological Association. 
Organizational Commitment 192

MacKinnon, D. P., Lockwood, C. M., \& Williams, J. (2004). Confidence limits for the indirect effect. Multivariate Behavioral Research, 39(1), 99-128.

Macey, W. H., \& Schneider, B. (2008). The meaning of employee engagement. Industrial and Organizational Psychology, 1(1), 3-30.

Manstead, A. S. R. (1996). Attitudes and behaviour. In G. R. Semin \& K. Fiedler (Eds.), (pp. 3-29). London: Sage.

Masterson, S. S., Lewis, K., Goldman, B. M., \& Taylor, M. S. (2000). Integrating justice and social exchange: The differing effects of fair procedures and treatment on work relationshipsIntegrating justice and social exchange: The differing effects of fair procedures and treatment on work relationships. The Academy of Management Journal, 43, 738-748. Academy of Management.

Mathieu, J. E., \& Zajac, D. M. (1990). A review and meta-analysis of the antecedents, correlates, and consequences of organizational commitment. Psychological Bulletin, 108, 171-194.

McCall, G. J., \& Simmons, J. L. (1978). Identities and interactions : an examination of human associations in everyday life. New York: Free Press.

McCaul, K. D., O’Neill, K. H., \& Glasgow, R. E. (1988). Predicting the performance of dental hygiene behaviors: An examination of the Fishbein and Ajzen model and self-efficacy expectations. Journal of Applied Social Psychology, 18, 114-128.

McDonald, D. J., \& Makin, P. J. (2000). The psychological contract, organisational commitment and job satisfaction of temporary staff. Leadership \& Organization Development Journal, 21, 84-91. 
Organizational Commitment 193

McGee, G. W., \& Ford, R. C. (1987). Two (or more?) dimensions of organizational commitment: Reexamination of the affective and continuance commitment scales. Journal of Applied Psychology, 72, 638-641.

McInnis, K. J., Meyer, J. P., \& Feldman, S. (2009). Psychological contracts and their implications for commitment: A feature-based approach. Journal of Vocational Behavior, 74, 165-180.

McMillan, B., \& Conner, M. (2003). Applying an extended version of the theory of planned behavior to illicit drug use among students. Journal of Applied Social Psychology, 33, 1662-1683.

Meyer, J. P., \& Allen, N. J. (1984). Testing the "side-bet theory" of organizational commitment: Some methodological considerations. Journal of Applied Psychology, 69, 372-378.

Meyer, J. P., \& Allen, N. J. (1991). A three-component conceptualization of organizational commitment. Human Resource Management Review, 1(1), 61-89.

Meyer, J. P., \& Allen, N. J. (1997). Commitment in the workplace : theory, research, and application. Thousand Oaks, Calif.: Sage Publications.

Meyer, J. P., \& Herscovitch, L. (2001). Commitment in the workplace: Toward a general model. Human Resource Management Review, 11, 299-326.

Meyer, J. P., \& Parfyonova, N. M. (2010). Normative commitment in the workplace: A theoretical analysis and re-conceptualization. Human Resource Management Review, 20, 283-294. 
Meyer, J. P., \& Smith, C. A. (2000). HRM practices and organizational commitment: Test of a mediation model. Canadian Journal of Administrative Sciences / Revue Canadienne des Sciences de l'Administration, 17, 319-331.

Meyer, J. P., Allen, N. J., \& Smith, C. A. (1993). Commitment to organizations and occupations: Extension and test of a three-component conceptualization. Journal of Applied Psychology, 78, 538-551.

Meyer, J. P., Paunonen, S. V., Gellatly, I. R., Goffin, R. D., \& Jackson, D. N. (1989). Organizational commitment and job performance: It's the nature of the commitment that counts. Journal of Applied Psychology, 74(1), 152-156.

Meyer, J. P., Stanley, D. J., Herscovitch, L., \& Topolnytsky, L. (2002). Affective, continuance, and normative commitment to the organization: A meta-analysis of antecedents, correlates, and consequences. Journal of Vocational Behavior, 61(1), $20-52$.

Millham, J. (1974). Two components of need for approval score and their relationship to cheating following success and failure. Journal of Research in Personality, 8, 378-392.

Morrow, P. C. (1993). The theory and measurement of work commitment. Greenwich, CA: JAI Press.

Morrow, Paula C. (1983). Concept redundancy in organizational research: The case of work commitment. The Academy of Management Review, 8, 486-500. Academy of Management. 
Organizational Commitment 195

Mowday, R. T., Porter, L. W., \& Steers, R. M. (1982). Employee-organization linkages : the psychology of commitment, absenteeism, and turnover. New York: Academic Press.

Mowday, R. T., Steers, R. M., \& Porter, L. W. (1979a). The measurement of organizational commitment. Journal of Vocational Behavior, 14, 224-247.

Mowday, R. T., Steers, R. M., \& Porter, L. W. (1979b). The measurement of organizational commitment. Journal of Vocational Behavior, 14, 224-247.

Muthén, L. K., \& Muthén, B. O. (2011). Mplus (Version 6.11) [Computer software]. Los Angeles, CA: Statmodel.

Myer, J. P., Becker, T. E., \& Vandenberghe, C. (2004). Employee commitment and motivation: A conceptual analysis and integrative model. Journal of Applied Psychology, 89, 991-1007.

Ng, T. W. H., \& Feldman, D. C. (2010). The relationships of age with job attitudes: A meta-analysis. Personnel Psychology, 63, 677-718.

Organ, D. W. (1988). OCB: The good soldier syndrome. Lexington Books, Lexington, MA.

Organ, Dennis W. (1997). Organizational citizenship behavior: It's construct clean-up time. Human Performance, 10, 85-97.

Osgood, C. E., Suci, G. J., \& Tannenbaum, P. H. (1957). The measurement of meaning. University of Illinois Press. 
O’Reilly, C. I., \& Chatman, J. (1986). Organizational commitment and psychological attachment: The effects of compliance, identification, and internalization on prosocial behavior. Journal of Applied Psychology, 71, 492-499.

Pare, G., \& Tremblay, M. (2007). The influence of high-involvement human resources practices, procedural justice, organizational commitment, and citizenship behaviors on information technology professionals' turnover intentions. Group \& Organization Management, 32, 326-357.

Penney, L. M., \& Spector, P. E. (2005). Job stress, incivility, and counterproductive work behavior (CWB): The moderating role of negative affectivity. Journal of Organizational Behavior, 26, 777-796.

Pfeffer, J., \& Lawler, J. (1980). Effects of job alternatives, extrinsic rewards, and behavioral commitment on attitude toward the organization: A field test of the insufficient justification paradigm. Administrative Science Quarterly, 25(1), 3856.

Podsakoff, P. M., MacKenzie, S. B., Lee, J.-Y., \& Podsakoff, N. P. (2003). Common method biases in behavioral research: A critical review of the literature and recommended remedies. Journal of Applied Psychology, 88, 879.

Pomazal, R. J., \& Jaccard, J. J. (1976). An informational approach to altruistic behavior. Journal of Personality and Social Psychology, 33, 317-326.

Porter, L. W., Steers, R. M., Mowday, R. T., \& Boulian, P. V. (1974). Organizational commitment, job satisfaction, and turnover among psychiatric technicians. Journal of Applied Psychology, 59, 603-609. 
Organizational Commitment 197

Powell, D. M., \& Meyer, J. P. (2004). Side-bet theory and the three-component model of organizational commitment. Journal of Vocational Behavior, 65(1), 157-177.

Preacher, K. J., \& Hayes, A. F. (2004). SPSS and SAS procedures for estimating indirect effects in simple mediation models. Behavior Research Methods, 36, 717-731.

Quick, J. C. (1999). Occupational health psychology: The convergence of health and clinical psychology with public health and preventive medicine in an organizational context. Professional Psychology - Research \& Practice, 30, 123128.

Randall, D. M., Fedor, D. B., \& Longenecker, C. O. (1990). The behavioral expression of organizational commitment. Journal of Vocational Behavior, 36, 210-224.

Reichers, A. E. (1985). A review and reconceptualization of organizational commitment. Academy of Management Review, 10, 465-476.

Rhoades, L., \& Eisenberger, R. (2002). Perceived organizational support: A review of the literature. Journal of Applied Psychology, 87, 698-714.

Ritzer, G., \& Trice, H. M. (1969). An Empirical Study of Howard Becker's Side-Bet Theory. Social Forces, 47, 475-478. University of North Carolina Press.

Rotter, J. B. (1966). Generalized expectancies for internal versus external control of reinforcement. Psychological Monographs: General and Applied, 80(1), 1-28.

Rotundo, M., \& Xie, J. L. (2008). Understanding the domain of counterproductive work behaviour in China. The International Journal of Human Resource Management, $19,856-877$. 
Rousseau, D. M. (1989). Psychological and implied contracts in organizations. Employee Responsibilities and Rights Journal, 2, 121-139.

Rudolph, K. D., Caldwell, M. S., \& Conley, C. S. (2005). Need for approval and children's well-being. Child Development, 76, 309-323.

Sackett, P. R., Berry, C. M., Wiemann, S. A., \& Laczo, R. M. (2006). Citizenship and counterproductive behavior: Clarifying relations between the two domains. Human Performance, 19, 441-464.

Scholl, R. W. (1981). Differentiating organizational commitment from expectancy as a motivating force. Academy of Management Review, 6, 589-599.

Settoon, R. P., Bennett, N., \& Liden, R. C. (1996). Social exchange in organizations: Perceived organizational support, leader-member exchange, and employee reciprocity. Journal of Applied Psychology, 81, 219-227.

Smetana, J. G., \& Adler, N. E. (1980). Fishbein's value x expectancy model: An examination of some assumptions. Pers Soc Psychol Bull, 6(1), 89-96.

Snell, S. A., \& James W. Dean, J. (1992). Integrated Manufacturing and Human Resource Management: A Human Capital Perspective. The Academy of Management Journal, 35, 467-504. Academy of Management.

Solinger, O. N., van Olffen, W., \& Roe, R. A. (2008). Beyond the three-component model of organizational commitment. Journal of Applied Psychology, 93(1), 7083. 
Organizational Commitment 199

Spector, P. E., \& Fox, S. (2002). An emotion-centered model of voluntary work behavior: Some parallels between counterproductive work behavior and organizational citizenship behavior. Human Resource Management Review, 12, 269-292.

Spector, P. E., \& Fox, S. (2005). A model of counterproductive work behavior. In S. Fox \& P. E. Spector (Eds.), (pp. 151-174). Washington, DC: American Psychological Association.

Spector, P. E., Fox, Suzy, Penney, L. M., Bruursema, K., Goh, A., \& Kessler, S. (2006). The dimensionality of counterproductivity: Are all counterproductive behaviors created equal? Journal of Vocational Behavior, 68, 446-460.

Stryker, S., \& Serpe, R. T. (1982). Commitment, identity salience and role Behavior. In W. Ickes \& E. Knowles (Eds.), Personality, Roles and Social Behavior (pp. 199218). New York: Springer-Verlag.

Terry, D. J., \& O’Leary, J. E. (1995). The theory of planned behaviour: The effects of perceived behavioural control and self-efficacy. British Journal of Social Psychology, 34, 199-220.

Thoresen, C. J., Kaplan, S. A., Barsky, A. P., Warren, C. R., \& de Chermont, K. (2003). The affective underpinnings of job perceptions and attitudes: A meta-analytic review and integration. Psychological Bulletin, 129, 914-945.

Ting-Toomey, S., Gao, G., Trubisky, P., Yang, Z., Kim, H. S., Lin, S.-L., \& Nishida, T. (1991). Culture, face maintenance, and styles of handling interpersonal conflicts: A study in five cultures. International Journal of Conflict Management, 2, 275296. 
Tinsley, C. H. (1998). Models of conflict resolution in Japanese, German, and American cultures. Journal of Applied Psychology, 83, 323-316.

Tinsley, C. H. (2001). How negotiators get to yes: predicting the constellation of strategies used across cultures to negotiate conflict. Journal of Applied Psychology, 86, 583-593.

Toneatto, T., \& Binik, Y. (1987). The role of intentions, social norms, and attitudes in the performance of dental flossing: A test of the theory of reasoned action. Journal of Applied Social Psychology, 17, 593-603.

Triandis, H. C. (1977). Interpersonal behavior. Monterey, CA: Brooks/Cole.

Triandis, H. C. (1980). Values, attitudes, and interpersonal behavior. In H. E. Howe, Jr. \& M. M. Page (Eds.), (pp. 195-259). Lincoln: University of Nebraska Press.

Vroom, V. H. (1964). Work and motivation. Oxford, England: Wiley.

Watson, D., Clark, L. A., \& Tellegen, A. (1988). Development and validation of brief measures of positive and negative affect: The PANAS scales. Journal of Personality \& Social Psychology, 54, 1063-1070.

Wayne, S. J, Shore, L. M, Bommer, W. H., \& Tetrick, L. E. (2002). The role of fair treatment and rewards in perceptions of organizational support and leadermember exchange. Journal of Applied Psychology, 87, 590-598.

Wayne, Sandy J., Shore, Lynn M., \& Liden, Robert C. (1997). Perceived organizational support and leader-member exchange: A social exchange perspective. The Academy of Management Journal, 40(1), 82-111. 
Whitbourne, S. K. (1996). The aging individual: Physical and psychological perspectives. New York: Springer.

Wicker, A. W. (1969). Attitudes versus actions: The relationship of verbal and overt behavioral responses to attitude objects. Journal of Social Issues, 25, 41-78.

Wiener, Y. (1982). Commitment in organizations: A normative view. The Academy of Management Review, 7, 418-428. 
Appendix: Study questionnaire

WAVE 1: EMPLOYEES

Demographic Information

1. Date of Birth:

2. Gender: Male Female

3. Year of education:

4. How many years and months of total work experience do you have?

5. How many years and months for you to work in this organization?

6. How many years and months for you to work on the current position?

7. Marital status (Circle the appropriate answer):

Single Married Widowed Divorced Partnered

8. Do you have children? Yes No

If you do, how many children do you have?

9. Income per month:

Less than $\$ 1500 \quad 1500 \quad 1500-3000 \quad 3000-5000 \quad 5000-8000 \quad 8000-10000$

More than 10000

10. Your department name

11. Your supervisor name

Organizational Commitment

Please indicate your level of agreement or disagreement with each statement by circling one of the seven alternatives next to each statement.

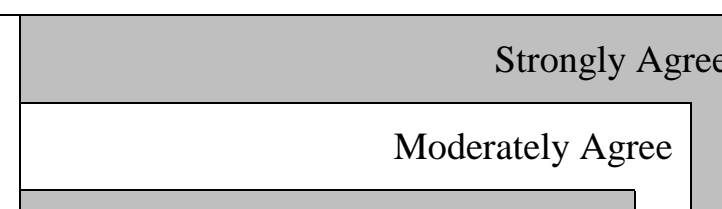

Slightly Agree

Neutral

Slightly Disagree

Moderately Disagree

Strongly Disagree

AC1. I would be very happy to spend the rest of my career at this 


\begin{tabular}{|c|c|c|c|c|c|c|c|}
\hline organization. & & & & & & & \\
\hline AC2. I really feel as if this organization's problems are my own. & 1 & 2 & 3 & 4 & 5 & 6 & 7 \\
\hline AC3. I do not feel like "part of the family" at my organization. & 1 & 2 & 3 & 4 & 5 & 6 & 7 \\
\hline AC4. I do not feel "emotionally attached" to this organization. & 1 & 2 & 3 & 4 & 5 & 6 & 7 \\
\hline AC5. This organization has a great deal of personal meaning for me. & 1 & 2 & 3 & 4 & 5 & 6 & 7 \\
\hline AC6. I do not feel a strong sense of belonging to my organization. & 1 & 2 & 3 & 4 & 5 & 6 & 7 \\
\hline $\begin{array}{l}\text { CC1. It would be very hard for me to leave my organization right } \\
\text { now, even if I wanted to. }\end{array}$ & 1 & 2 & 3 & 4 & 5 & 6 & 7 \\
\hline $\begin{array}{l}\text { CC2. Too much of my life would be disrupted if I decided I wanted } \\
\text { to leave my organization right now. }\end{array}$ & 1 & 2 & 3 & 4 & 5 & 6 & 7 \\
\hline $\begin{array}{l}\text { CC3. Right now, staying with my organization is a matter of } \\
\text { necessity as much as desire. }\end{array}$ & 1 & 2 & 3 & 4 & 5 & 6 & 7 \\
\hline $\begin{array}{l}\text { CC4. I believe that I have too few options to consider leaving this } \\
\text { organization. }\end{array}$ & 1 & 2 & 3 & 4 & 5 & 6 & 7 \\
\hline $\begin{array}{l}\text { CC5. One of the few negative consequences of leaving this } \\
\text { organization would be the scarcity of available alternatives. }\end{array}$ & 1 & 2 & 3 & 4 & 5 & 6 & 7 \\
\hline $\begin{array}{l}\text { CC6. One of the major reasons I continue to work for this } \\
\text { organization is that leaving would require considerable } \\
\text { personal sacrifice; another organization may not match the } \\
\text { overall benefits I have here. }\end{array}$ & 1 & 2 & 3 & 4 & 5 & 6 & 7 \\
\hline $\begin{array}{l}\text { CC7. If I had not already put so much of myself into this } \\
\text { organization, I might consider working elsewhere. }\end{array}$ & 1 & 2 & 3 & 4 & 5 & 6 & 7 \\
\hline $\begin{array}{l}\text { NC1. I do not feel any obligation to remain with my current } \\
\text { employer. }\end{array}$ & 1 & 2 & 3 & 4 & 5 & 6 & 7 \\
\hline $\begin{array}{l}\text { NC2. Even if it were to my advantage, I do not feel it would be right } \\
\text { to leave my organization now. }\end{array}$ & 1 & 2 & 3 & 4 & 5 & 6 & 7 \\
\hline NC3. I would feel guilty if I left my organization now. & 1 & 2 & 3 & 4 & 5 & 6 & 7 \\
\hline NC4. This organization deserves my loyalty. & 1 & 2 & 3 & 4 & 5 & 6 & 7 \\
\hline $\begin{array}{l}\text { NC5. I would not leave my organization right now because I have a } \\
\text { sense of obligation to the people in it. }\end{array}$ & 1 & 2 & 3 & 4 & 5 & 6 & 7 \\
\hline
\end{tabular}


NC6. I owe a great deal to my organization. \begin{tabular}{l|l|l|l|l|l|l|}
1 & 2 & 3 & 4 & 5 & 6 & 7
\end{tabular}

Utilitarian Expectancies

Constructive Voice

\begin{abstract}
People deal with the problems or problematic events that they encounter at work (e.g., difference of opinion with supervisor, frustrations with regard to the behavior of coworkers, or dissatisfaction with working schedule) in different ways depending on the outcomes (i.e., good or bad) of such behaviors to them. Please indicate your level of agreement or disagreement with the following statements below by circling one of the seven alternatives next to each statement. We are interested in your own assessment of such statement within your own organization and there is no right or wrong answer.
\end{abstract}

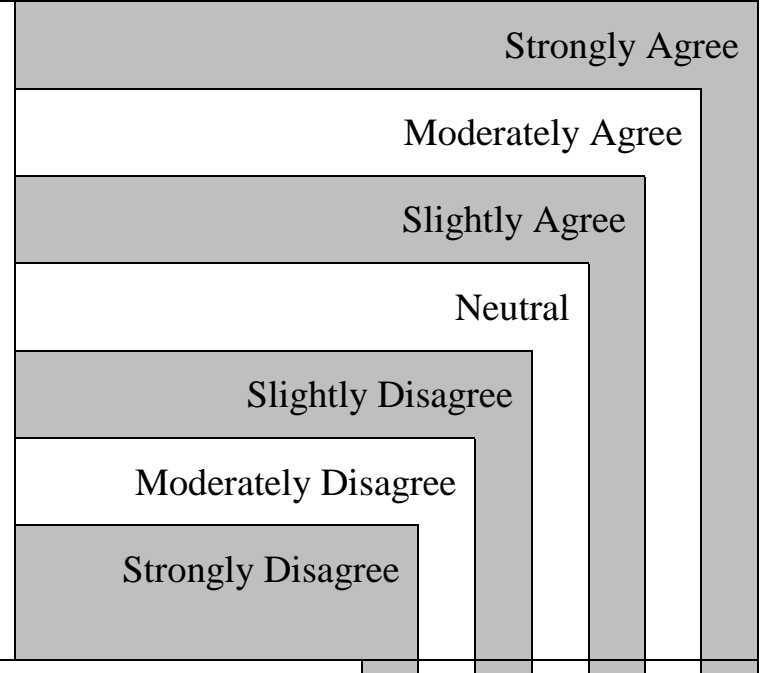

UECV1. I believe that trying to come to an understanding with parties related to the problem will yield good result for me

UECV2. I believe that, in collaboration with related parties in the organization, trying to find a solution that is satisfactory to everybody will bring good outcomes to me

UECV3. I believe that trying to work out an ideal solution in collaboration with related parties will be good for me

UECV4. I believe that, together with related parties in the organization, explore each other's opinions until the problems are solved will be beneficial for me

UECV5. I believe that trying to compromise with related parties in the organization in dealing with organizational problems will be good for me

UECV6. I believe that talking with related parties in the organization about the problem until a total agreement is reached will yield good result for me

UECV7. I believe that suggesting solutions to related parties in the organization in dealing with organizational problems will bring positive outcomes to me

UECV8. I believe that immediately reporting the problem to 


\begin{tabular}{|c|c|c|c|c|c|c|c|c|}
\hline & $\begin{array}{l}\text { supervisor and related parties in the organization will bring } \\
\text { good outcomes to me }\end{array}$ & & & & & & & \\
\hline UECV9. & $\begin{array}{l}\text { I believe that immediately trying to find a solution will } \\
\text { bring good outcomes to me }\end{array}$ & 1 & 2 & 3 & 4 & 5 & 6 & 7 \\
\hline UECV10. & $\begin{array}{l}\text { I believe that trying to think of different solutions to the } \\
\text { problem will be helpful for me }\end{array}$ & 1 & 2 & 3 & 4 & 5 & 6 & 7 \\
\hline UECV11. & $\begin{array}{l}\text { I believe that asking related parties in the organization for } \\
\text { compromises will yield good result for me }\end{array}$ & 1 & 2 & 3 & 4 & 5 & 6 & 7 \\
\hline
\end{tabular}

\section{Production Deviance}

\begin{tabular}{|c|c|c|c|c|c|c|c|c|}
\hline \multirow{7}{*}{$\begin{array}{l}\text { Under certain circumstances when people feel } \\
\text { stressful and/or feel that they are treated unfairly } \\
\text { and/or disrespectfully, they may intentionally do } \\
\text { harm to the organization depending on the } \\
\text { outcomes (i.e., good or bad) of such behaviors to } \\
\text { them. Please indicate your level of agreement or } \\
\text { disagreement with the following statements } \\
\text { below by circling one of the seven alternatives } \\
\text { next to each statement. We are interested in your } \\
\text { own assessment of such statement within your } \\
\text { own organization and there is no right or wrong } \\
\text { answer. }\end{array}$} & & \multicolumn{7}{|c|}{ Strongly Agree } \\
\hline & & \multicolumn{6}{|c|}{ Moderately Agree } & \\
\hline & & \multicolumn{5}{|c|}{ Slightly Agree } & & \\
\hline & & \multicolumn{5}{|c|}{ Neutral } & & \\
\hline & \multicolumn{5}{|c|}{ Slightly Disagree } & & & \\
\hline & \multicolumn{4}{|c|}{ Moderately Disagree } & & & & \\
\hline & \multicolumn{3}{|c|}{ Strongly Disagree } & & & & & \\
\hline \multicolumn{2}{|c|}{$\begin{array}{l}\text { UEPD1. I believe that sometimes doing my work incorrectly on } \\
\text { purpose will bring good outcomes to me. }\end{array}$} & 1 & 2 & 3 & 4 & 5 & 6 & 7 \\
\hline UEPD2. & $\begin{array}{l}\text { ourpose when } \\
\text { for me. }\end{array}$ & 1 & 2 & 3 & 4 & 5 & 6 & 7 \\
\hline $\begin{array}{l}\text { UEPD3. I believe that sometimes failing to foll } \\
\text { purpose will be good for me. }\end{array}$ & tructions on & 1 & 2 & 3 & 4 & 5 & 6 & 7 \\
\hline $\begin{array}{l}\text { UEPD4. I believe that sometimes giving obscu } \\
\text { information to others will be good for }\end{array}$ & uctions or & 1 & 2 & 3 & 4 & 5 & 6 & 7 \\
\hline $\begin{array}{l}\text { UEPD5. I believe that sometimes not providing } \\
\text { know things can go wrong later will b }\end{array}$ & $\begin{array}{l}\text { ing when I } \\
\text { ficial for me }\end{array}$ & 1 & 2 & 3 & 4 & 5 & 6 & 7 \\
\hline
\end{tabular}




\section{Behavioral Engagement}

\begin{tabular}{|c|c|c|c|c|c|c|c|c|}
\hline \multirow{7}{*}{$\begin{array}{l}\text { Facing with organizational challenges and } \\
\text { opportunities, people may decide to proactively } \\
\text { go beyond what is expected and exert extra } \\
\text { efforts and energy to meet new demands at work } \\
\text { depending on the outcomes (i.e., good or bad) of } \\
\text { such behaviors to them. Please indicate your } \\
\text { level of agreement or disagreement with the } \\
\text { following statements below by circling one of the } \\
\text { seven alternatives next to each statement. We are } \\
\text { interested in your own assessment of such } \\
\text { statement within your own organization and } \\
\text { there is no right or wrong answer. }\end{array}$} & & \multicolumn{7}{|c|}{ Strongly Agree } \\
\hline & & \multicolumn{7}{|c|}{ Moderately Agree } \\
\hline & & \multicolumn{6}{|c|}{ Slightly Agree } & \\
\hline & & \multicolumn{5}{|c|}{ Neutral } & & \\
\hline & \multicolumn{5}{|c|}{ Slightly Disagree } & & & \\
\hline & \multicolumn{4}{|c|}{ Moderately Disagree } & & & & \\
\hline & \multicolumn{3}{|c|}{ Strongly Disagree } & & & & & \\
\hline \multicolumn{2}{|c|}{$\begin{array}{l}\text { UEBE1. I believe that working extra hour to solve organizational } \\
\text { problems will bring good outcomes to me }\end{array}$} & 1 & 2 & 3 & 4 & 5 & 6 & 7 \\
\hline \multicolumn{2}{|c|}{$\begin{array}{l}\text { UEBE2. I believe that working extra hour to meet additional } \\
\text { demand of the job will yield good result for me }\end{array}$} & 1 & 2 & 3 & 4 & 5 & 6 & 7 \\
\hline \multicolumn{2}{|c|}{$\begin{array}{l}\text { UEBE3. I believe that doing extra works which beyond my } \\
\text { responsibility will be good for me }\end{array}$} & 1 & 2 & 3 & 4 & 5 & 6 & 7 \\
\hline \multicolumn{2}{|c|}{$\begin{array}{l}\text { UEBE4. I believe that taking extra responsibility to deal will new } \\
\text { organizational challenges will be beneficial for me }\end{array}$} & 1 & 2 & 3 & 4 & 5 & 6 & 7 \\
\hline \multicolumn{2}{|c|}{$\begin{array}{l}\text { UEBE5. I believe that proactively seeking a better way of doing the } \\
\text { job will yield good result for me }\end{array}$} & 1 & 2 & 3 & 4 & 5 & 6 & 7 \\
\hline $\begin{array}{l}\text { UEBE6. I believe that taking my own risk in i } \\
\text { doing things will be good for me }\end{array}$ & ig new ways of & 1 & 2 & 3 & 4 & 5 & 6 & 7 \\
\hline $\begin{array}{l}\text { UEBE7. I believe that supporting new ideas th } \\
\text { comparative advantages to the organi } \\
\text { beneficial for me }\end{array}$ & $\begin{array}{l}\text { lieve will bring } \\
\text { will be }\end{array}$ & 1 & 2 & 3 & 4 & 5 & 6 & 7 \\
\hline $\begin{array}{l}\text { UEBE8. I believe that actively monitoring the } \\
\text { respond quickly to changes will bring }\end{array}$ & $\begin{array}{l}\text { environment to } \\
\text { outcomes to me }\end{array}$ & 1 & 2 & 3 & 4 & 5 & 6 & 7 \\
\hline
\end{tabular}


Normative Expectancies

Constructive Voice

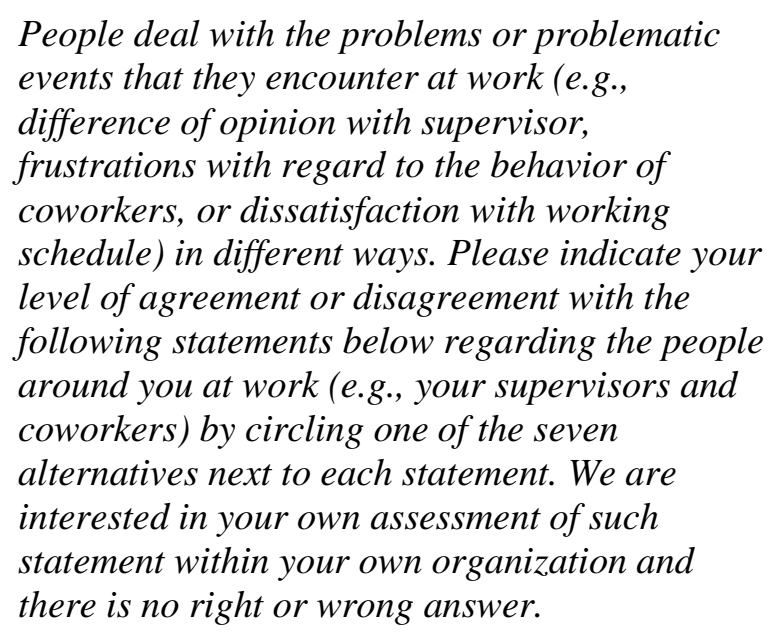

NECV1. People around me at work (e.g., my supervisors and coworkers) usually try to come to an understanding with parties related to the problem

NECV2. People around me at work (e.g., my supervisors and coworkers) usually, in collaboration with related parties in the organization, try to find a solution that is satisfactory to everybody

NECV3. People around me at work (e.g., my supervisors and coworkers) usually try to work out an ideal solution in collaboration with related parities

NECV4. People around me at work (e.g., my supervisors and coworkers) usually, together with related parties in the organization, explore each other's opinions until the problems are solved

NECV5. People around me at work (e.g., my supervisors and coworkers) usually try to compromise with related parties in the organization in dealing organizational problems

NECV6. People around me at work (e.g., my supervisors and coworkers) usually talk with related parties in the organization about the problem until a total agreement is reached

NECV7. People around me at work (e.g., my supervisors and coworkers) usually suggest solutions to related parties in
Strongly Agree

Moderately Agree

Slightly Agree

Neutral

Slightly Disagree

rately Disagree

ly Disagree 


\begin{tabular}{|c|c|c|c|c|c|c|c|c|}
\hline & the organization in dealing with organizational problems & & & & & & & \\
\hline NECV8. & $\begin{array}{l}\text { People around me at work (e.g., my supervisors and } \\
\text { coworkers) usually report the problem to supervisor and } \\
\text { related parties in the organization immediately }\end{array}$ & 1 & 2 & 3 & 4 & 5 & 6 & 7 \\
\hline NECV9. & $\begin{array}{l}\text { People around me at work (e.g., my supervisors and } \\
\text { coworkers) usually try to find a solution immediately }\end{array}$ & 1 & 2 & 3 & 4 & 5 & 6 & 7 \\
\hline NECV10. & $\begin{array}{l}\text { People around me at work (e.g., my supervisors and } \\
\text { coworkers) usually try to think of different solutions to the } \\
\text { problem }\end{array}$ & 1 & 2 & 3 & 4 & 5 & 6 & 7 \\
\hline NECV11. & $\begin{array}{l}\text { People around me at work (e.g., my supervisors and } \\
\text { coworkers) usually ask related parties in the organization } \\
\text { for compromises }\end{array}$ & 1 & 2 & 3 & 4 & 5 & 6 & 7 \\
\hline
\end{tabular}

Production Deviance

\begin{tabular}{|c|c|c|c|c|c|c|c|c|}
\hline \multirow{7}{*}{$\begin{array}{l}\text { Under certain circumstances when people feel } \\
\text { stressful and/or feel that they are treated unfairly } \\
\text { and/or disrespectfully, they may intentionally do } \\
\text { harm to the organization. Please indicate your } \\
\text { level of agreement or disagreement with the } \\
\text { following statements below regarding the people } \\
\text { around you at work (e.g., your supervisors and } \\
\text { coworkers) by circling one of the seven } \\
\text { alternatives next to each statement. We are } \\
\text { interested in your own assessment of such } \\
\text { statement within your own organization and } \\
\text { there is no right or wrong answer. }\end{array}$} & & \multicolumn{7}{|c|}{ Strongly Agree } \\
\hline & & \multicolumn{7}{|c|}{ Moderately Agree } \\
\hline & & \multicolumn{5}{|c|}{ Slightly Agree } & & \\
\hline & & \multicolumn{5}{|c|}{ Neutral } & & \\
\hline & \multicolumn{5}{|c|}{ Slightly Disagree } & & & \\
\hline & \multicolumn{4}{|c|}{ Moderately Disagree } & & & & \\
\hline & \multicolumn{3}{|c|}{ Strongly Disagree } & & & & & \\
\hline NEPD1. & $\begin{array}{l}\text { sors and } \\
\text { ctly on purpose }\end{array}$ & 1 & 2 & 3 & 4 & 5 & 6 & 7 \\
\hline \multicolumn{2}{|c|}{$\begin{array}{l}\text { NEPD2. People around me at work (e.g., my supervisors and } \\
\text { coworkers) sometimes work slowly on purpose when } \\
\text { things need to get done }\end{array}$} & 1 & 2 & 3 & 4 & 5 & 6 & 7 \\
\hline \multicolumn{2}{|c|}{$\begin{array}{l}\text { NEPD3. People around me at work (e.g., my supervisors and } \\
\text { coworkers) sometimes fail to follow instructions on } \\
\text { purpose }\end{array}$} & 1 & 2 & 3 & 4 & 5 & 6 & 7 \\
\hline $\begin{array}{l}\text { NEPD4. People around me at work (e.g., my st } \\
\text { coworkers) sometimes give obscure ir } \\
\text { information to others }\end{array}$ & $\begin{array}{l}\text { isors and } \\
\text { tions or }\end{array}$ & 1 & 2 & 3 & 4 & J & 0 & 7 \\
\hline
\end{tabular}


NEPD5. People around me at work (e.g., my supervisors and coworkers) sometimes do not provide warning when they know things can go wrong later

\section{Behavioral Engagement}
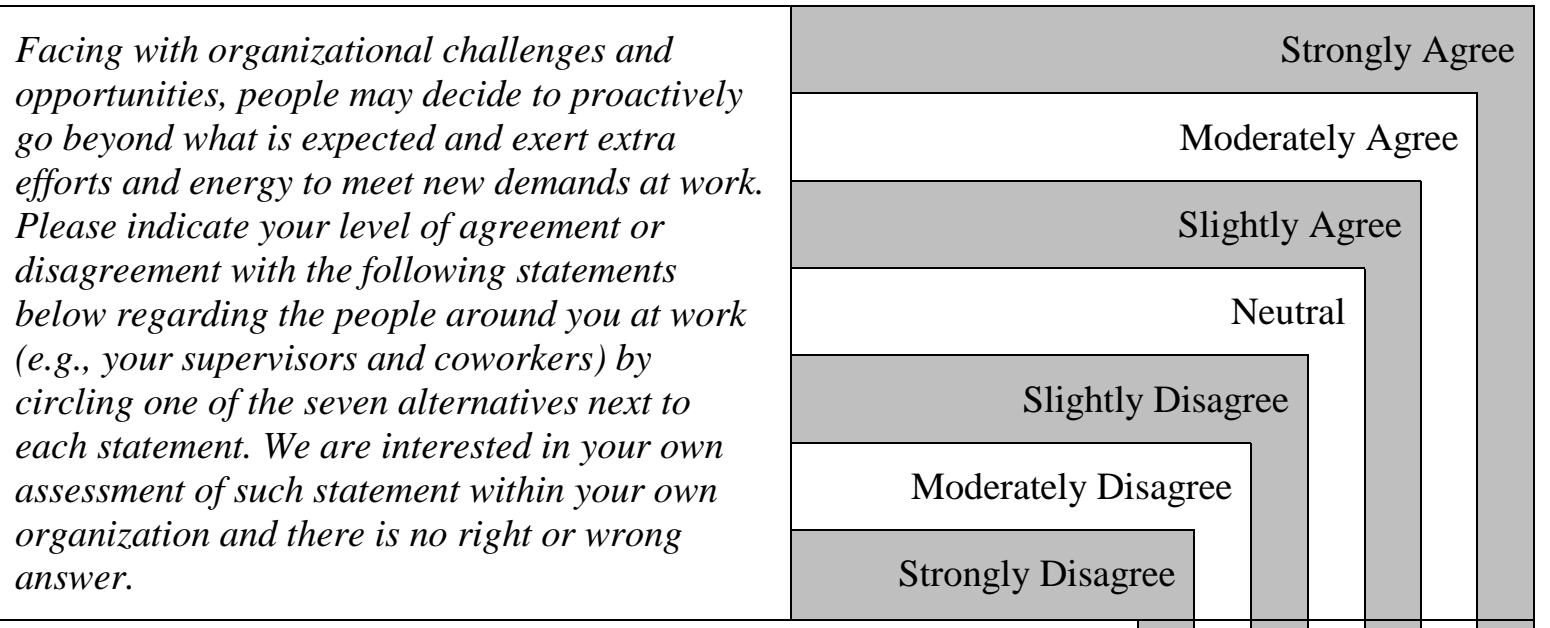

NEBE1. People around me at work (e.g., my supervisors and \begin{tabular}{ll|l|l|l|l|l|l|l|} 
coworkers) are usually willing to work extra hour to solve & 1 & 2 & 3 & 4 & 5 & 6 & 7
\end{tabular} organizational problems

NEBE2. People around me at work (e.g., my supervisors and coworkers) are usually willing to work extra hour to meet additional demand of the job

NEBE3. People around me at work (e.g., my supervisors and coworkers) are usually willing to do extra works which beyond their responsibility

NEBE4. People around me at work (e.g., my supervisors and coworkers) are usually willing to take extra responsibility to deal with new organizational challenges

NEBE5. People around me at work (e.g., my supervisors and coworkers) usually proactively seek a better way of doing the job

NEBE6. People around me at work (e.g., my supervisors and coworkers) usually take their own risk in initiating new ways of doing things

NEBE7. People around me at work (e.g., my supervisors and coworkers) usually support new ideas that they believe will bring comparative advantages to the organization 
NEBE8. People around me at work (e.g., my supervisors and coworkers) usually monitor the work environment actively to respond quickly to changes

Self-Identity Expectancies

Constructive Voice

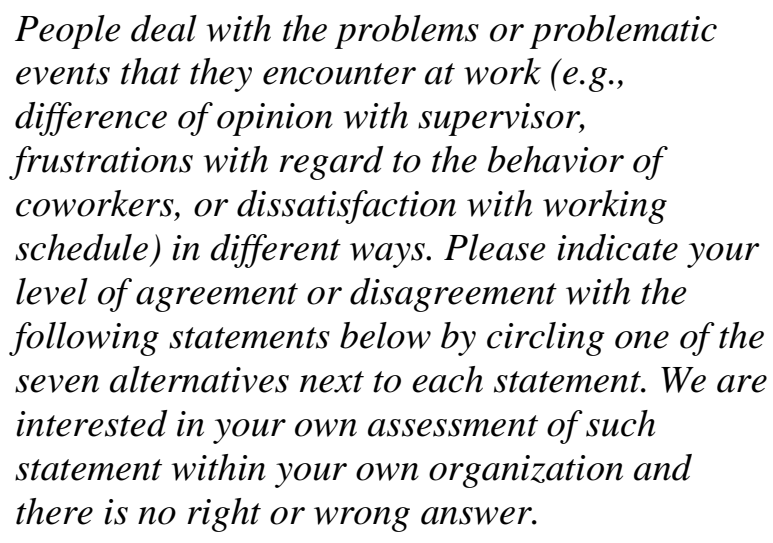

People deal with the problems or problematic events that they encounter at work (e.g., difference of opinion with supervisor, frustrations with regard to the behavior of coworkers, or dissatisfaction with working schedule) in different ways. Please indicate your level of agreement or disagreement with the following statements below by circling one of the seven alternatives next to each statement. We are interested in your own assessment of such statement within your own organization and there is no right or wrong answer.

SECV1. Trying to come to an understanding with parties related to the problem makes me feel as a good member of the organization

SECV2. In collaboration with related parties in the organization, trying to find a solution that is satisfactory to everybody makes me feel as a good member of the organization

SECV3. Trying to work out an ideal solution in collaboration with related parities makes me feel as a good member of the organization

SECV4. Together with related parties in the organization, exploring each other's opinions until the problems are solved makes me feel as a good member of the organization

SECV5. Trying to compromise with related parties in the organization in dealing with organizational problems makes me feel as a good member of the organization

SECV6. Talking with related parties in the organization about the problem until a total agreement is reached makes me feel as a good member of the organization

SECV7. Suggesting solutions to related parties in the organization in dealing with organizational problems makes me feel as a
Strongly Agree

Moderately Agree

Slightly Agree

Neutral

lightly Disagree

Moderately Disagree

Strongly Disagree 


\begin{tabular}{|c|c|c|c|c|c|c|c|c|}
\hline & good member of the organization & & & & & & & \\
\hline SECV8. & $\begin{array}{l}\text { Immediately reporting the problem to supervisor and } \\
\text { related parties in the organization makes me feel as a good } \\
\text { member of the organization }\end{array}$ & 1 & 2 & 3 & 4 & 5 & 6 & 7 \\
\hline SECV9. & $\begin{array}{l}\text { Immediately trying to find a solution makes me feel as a } \\
\text { good member of the organization }\end{array}$ & 1 & 2 & 3 & 4 & 5 & 6 & 7 \\
\hline SECV10. & $\begin{array}{l}\text { Trying to think of different solutions to the problem makes } \\
\text { me feel as a good member of the organization }\end{array}$ & 1 & 2 & 3 & 4 & 5 & 6 & 7 \\
\hline SECV11. & $\begin{array}{l}\text { Asking related parties in the organization for compromises } \\
\text { makes me feel as a good member of the organization }\end{array}$ & 1 & 2 & 3 & 4 & 5 & 6 & 7 \\
\hline
\end{tabular}

\section{Production Deviance}

\begin{tabular}{|c|c|c|c|c|c|c|c|c|}
\hline \multirow{7}{*}{$\begin{array}{l}\text { Under certain circumstances when people feel } \\
\text { stressful and/or feel that they are treated unfairly } \\
\text { and/or disrespectfully, they may intentionally do } \\
\text { harm to the organization. Please indicate your } \\
\text { level of agreement or disagreement with the } \\
\text { following statements below by circling one of the } \\
\text { seven alternatives next to each statement. We are } \\
\text { interested in your own assessment of such } \\
\text { statement within your own organization and } \\
\text { there is no right or wrong answer. }\end{array}$} & & \multicolumn{7}{|c|}{ Strongly Agree } \\
\hline & & \multicolumn{7}{|c|}{ Moderately Agree } \\
\hline & & \multicolumn{5}{|c|}{ Slightly Agree } & & \\
\hline & & \multicolumn{5}{|c|}{ Neutral } & & \\
\hline & \multicolumn{5}{|c|}{ Slightly Disagree } & & & \\
\hline & \multicolumn{4}{|c|}{ Moderately Disagree } & & & & \\
\hline & \multicolumn{3}{|c|}{ Strongly Disagree } & & & & & \\
\hline \multicolumn{2}{|c|}{$\begin{array}{l}\text { Purposely doing your work incorrectly makes me feel as a } \\
\text { bad member of the organization }\end{array}$} & 1 & 2 & 3 & 4 & 5 & 6 & 7 \\
\hline \multicolumn{2}{|c|}{$\begin{array}{l}\text { Purposely working slowly when things need to get done } \\
\text { makes me feel as a bad member of the organization }\end{array}$} & 1 & 2 & 3 & 4 & 5 & 6 & 7 \\
\hline \multicolumn{2}{|c|}{$\begin{array}{l}\text { Purposely failing to follow instructions makes me feel as a } \\
\text { bad member of the organization }\end{array}$} & 1 & 2 & 3 & 4 & 5 & 6 & 7 \\
\hline \multicolumn{2}{|c|}{$\begin{array}{l}\text { Giving obscure instructions or information to others makes } \\
\text { me feel as a bad member of the organization }\end{array}$} & 1 & 2 & 3 & 4 & 5 & 6 & 7 \\
\hline \multicolumn{2}{|c|}{$\begin{array}{l}\text { Not providing warning when you know things can go } \\
\text { wrong later makes me feel as a bad member of the } \\
\text { organization }\end{array}$} & 1 & 2 & 3 & 4 & 5 & 6 & \\
\hline
\end{tabular}




\section{Behavioral Engagement}

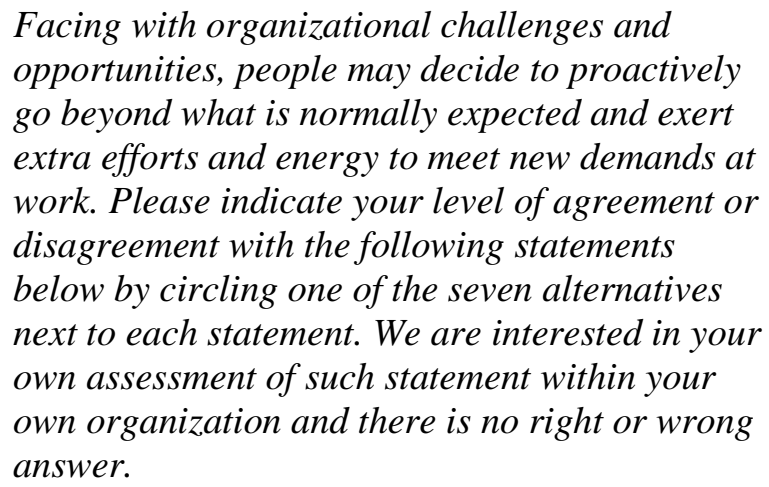

Facing with organizational challenges and opportunities, people may decide to proactively go beyond what is normally expected and exert extra efforts and energy to meet new demands at work. Please indicate your level of agreement or disagreement with the following statements below by circling one of the seven alternatives next to each statement. We are interested in your own assessment of such statement within your own organization and there is no right or wrong answer.

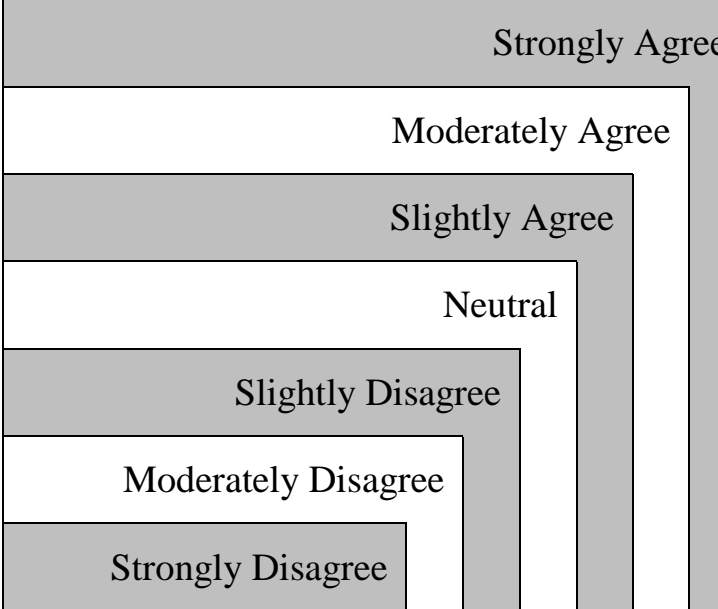

Strongly Disagree

SEBE1. Working extra hour to solve organizational problems makes me feel as a good member of the organization

SEBE2. Working extra hour to meet additional demand of the job makes me feel as a good member of the organization

SEBE3. Doing extra works which beyond one's responsibility makes me feel as a good member of the organization

SEBE4. Taking extra responsibility to deal with new organizational challenges makes me feel as a good member of the organization

SEBE5. Proactively seeking a better way of doing the job makes me feel as a good member of the organization

SEBE6. Taking your own risk in initiating new ways of doing
things makes me feel as a good member of the organization

SEBE6. Taking your own risk in initiating new ways of doing
things makes me feel as a good member of the organization

SEBE7. Supporting new ideas that you believe will bring comparative advantages to the organization

SEBE8. Actively monitoring the work environment to respond quickly to changes makes me feel as a good member of the organization 
Attitude toward behaviors (Bad - Good Dimension)

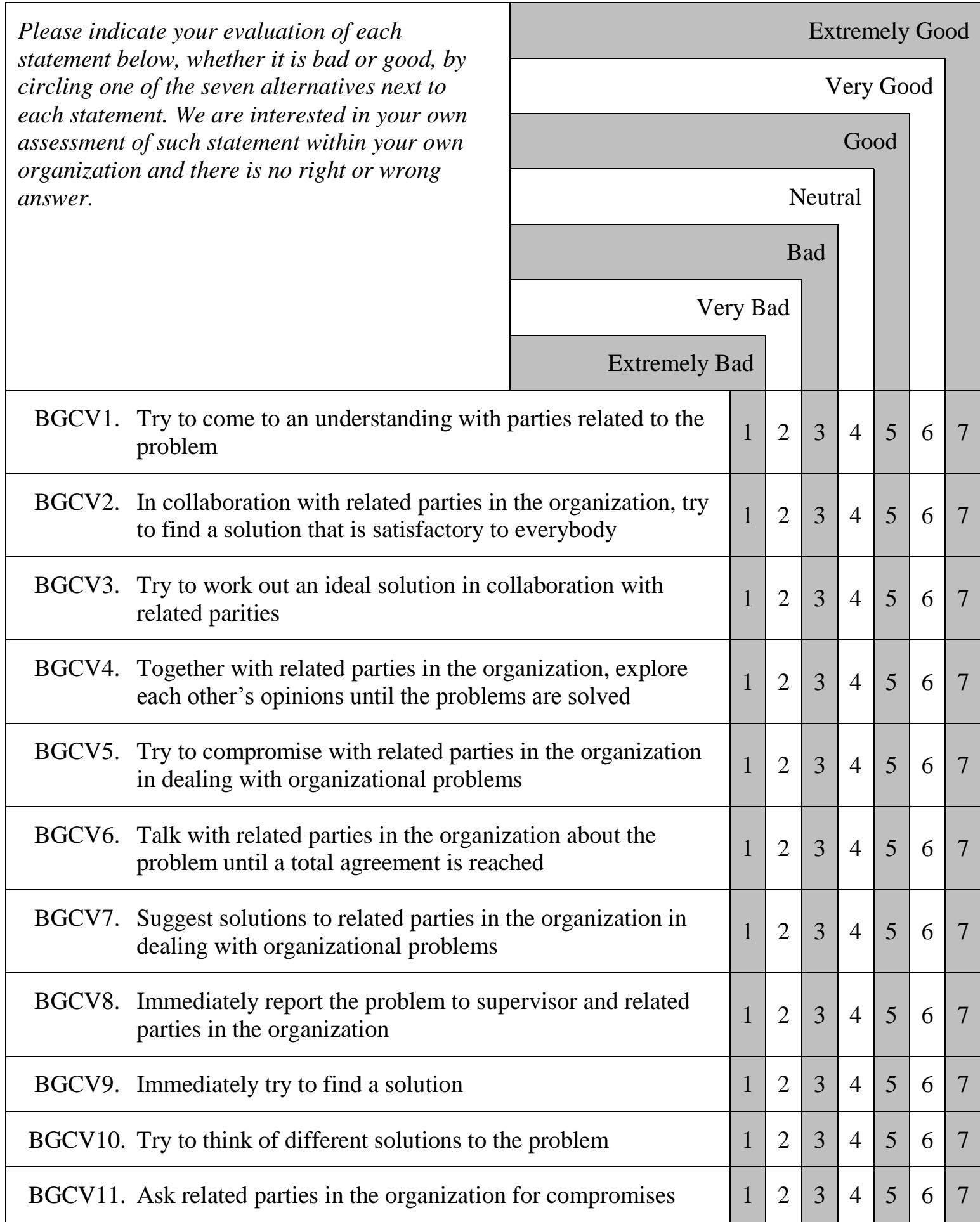




\begin{tabular}{|c|c|c|c|c|c|c|c|}
\hline BGPD1. Purposely do your work incorrectly & 1 & 2 & 3 & 4 & 5 & 6 & 7 \\
\hline BGPD2. Purposely work slowly when things need to get done & 1 & 2 & 3 & 4 & 5 & 6 & 7 \\
\hline BGPD3. Purposely fail to follow instructions & 1 & 2 & 3 & 4 & 5 & 6 & 7 \\
\hline BGPD4. Give obscure instructions or information to others & 1 & 2 & 3 & 4 & 5 & 6 & 7 \\
\hline $\begin{array}{l}\text { BGPD5. Do not provide warning when you know things can go } \\
\text { wrong later }\end{array}$ & 1 & 2 & 3 & 4 & 5 & 6 & 7 \\
\hline $\begin{array}{l}\text { BGBE1. Willing to work extra hour to solve organizational } \\
\text { problems/ }\end{array}$ & 1 & 2 & 3 & 4 & 5 & 6 & 7 \\
\hline $\begin{array}{l}\text { BGBE2. Willing to work extra hour to meet additional demand of } \\
\text { the job }\end{array}$ & 1 & 2 & 3 & 4 & 5 & 6 & 7 \\
\hline $\begin{array}{l}\text { BGBE3. Willing to do extra works which beyond one's } \\
\text { responsibility }\end{array}$ & 1 & 2 & 3 & 4 & 5 & 6 & 7 \\
\hline $\begin{array}{l}\text { BGBE4. Willing to take extra responsibility to deal with new } \\
\text { organizational challenges }\end{array}$ & 1 & 2 & 3 & 4 & 5 & 6 & 7 \\
\hline BGBE5. Proactively seek a better way of doing the job & 1 & 2 & 3 & 4 & 5 & 6 & 7 \\
\hline BGBE6. Take your own risk in initiating new ways of doing things & 1 & 2 & 3 & 4 & 5 & 6 & 7 \\
\hline $\begin{array}{l}\text { BGBE7. Support new ideas that you believe will bring comparative } \\
\text { advantages to the organization }\end{array}$ & 1 & 2 & 3 & 4 & 5 & 6 & 7 \\
\hline $\begin{array}{l}\text { BGBE8. Actively monitor the work environment to respond quickly } \\
\text { to changes }\end{array}$ & 1 & 2 & 3 & 4 & 5 & 6 & 7 \\
\hline
\end{tabular}

Attitude toward behaviors (Harmful - Beneficial Dimension)

Please indicate your evaluation of each statement below, whether it is harmful or beneficial, by circling one of the seven alternatives next to each statement. We are interested in your own assessment of such statement within your own organization and there is no right or wrong answer.

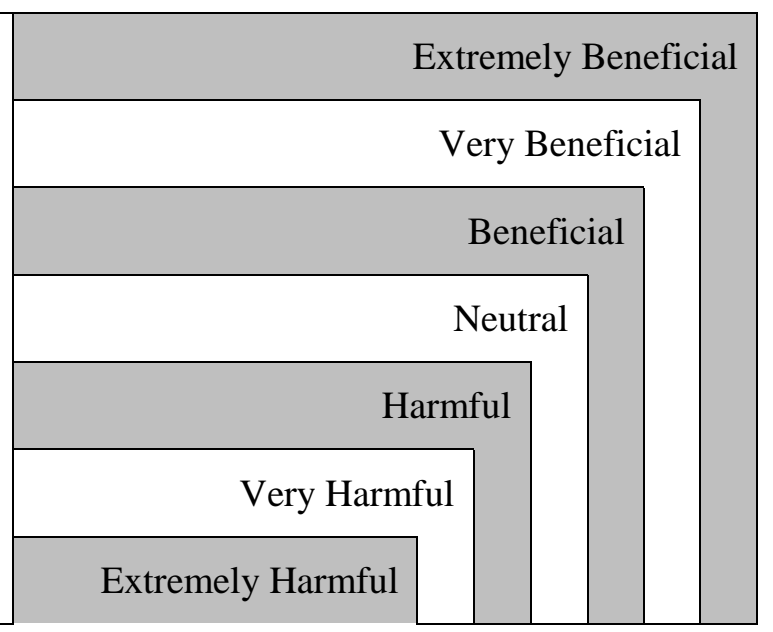




\begin{tabular}{|c|c|c|c|c|c|c|c|c|}
\hline HBCV1. & $\begin{array}{l}\text { Try to come to an understanding with parties related to the } \\
\text { problem }\end{array}$ & 1 & 2 & 3 & 4 & 5 & 6 & 7 \\
\hline HBCV2. & $\begin{array}{l}\text { In collaboration with related parties in the organization, try } \\
\text { to find a solution that is satisfactory to everybody }\end{array}$ & 1 & 2 & 3 & 4 & 5 & 6 & 7 \\
\hline HBCV3. & $\begin{array}{l}\text { Try to work out an ideal solution in collaboration with } \\
\text { related parities }\end{array}$ & 1 & 2 & 3 & 4 & 5 & 6 & 7 \\
\hline HBCV4. & $\begin{array}{l}\text { Together with related parties in the organization, explore } \\
\text { each other's opinions until the problems are solved }\end{array}$ & 1 & 2 & 3 & 4 & 5 & 6 & 7 \\
\hline HBCV5. & $\begin{array}{l}\text { Try to compromise with related parties in the organization } \\
\text { in dealing with organizational problems }\end{array}$ & 1 & 2 & 3 & 4 & 5 & 6 & 7 \\
\hline HBCV6. & $\begin{array}{l}\text { Talk with related parties in the organization about the } \\
\text { problem until a total agreement is reached }\end{array}$ & 1 & 2 & 3 & 4 & 5 & 6 & 7 \\
\hline HBCV7. & $\begin{array}{l}\text { Suggest solutions to related parties in the organization in } \\
\text { dealing with organizational problems }\end{array}$ & 1 & 2 & 3 & 4 & 5 & 6 & 7 \\
\hline HBCV8. & $\begin{array}{l}\text { Immediately report the problem to supervisor and related } \\
\text { parties in the organization }\end{array}$ & 1 & 2 & 3 & 4 & 5 & 6 & 7 \\
\hline HBCV9. & Immediately try to find a solution & 1 & 2 & 3 & 4 & 5 & 6 & 7 \\
\hline HBCV10. & Try to think of different solutions to the problem & 1 & 2 & 3 & 4 & 5 & 6 & 7 \\
\hline HBCV11. & Ask related parties in the organization for compromises & 1 & 2 & 3 & 4 & 5 & 6 & 7 \\
\hline HBPD1. & Purposely do your work incorrectly & 1 & 2 & 3 & 4 & 5 & 6 & 7 \\
\hline HBPD2. & Purposely work slowly when things need to get done & 1 & 2 & 3 & 4 & 5 & 6 & 7 \\
\hline HBPD3. & Purposely fail to follow instructions & 1 & 2 & 3 & 4 & 5 & 6 & 7 \\
\hline HBPD4. & Give obscure instructions or information to others & 1 & 2 & 3 & 4 & 5 & 6 & 7 \\
\hline HBPD5. & $\begin{array}{l}\text { Do not provide warning when you know things can go } \\
\text { wrong later }\end{array}$ & 1 & 2 & 3 & 4 & 5 & 6 & 7 \\
\hline HBBE1. & $\begin{array}{l}\text { Willing to work extra hour to solve organizational } \\
\text { problems }\end{array}$ & 1 & 2 & 3 & 4 & 5 & 6 & 7 \\
\hline HBBE2. & $\begin{array}{l}\text { Willing to work extra hour to meet additional demand of } \\
\text { the job }\end{array}$ & 1 & 2 & 3 & 4 & 5 & 6 & 7 \\
\hline HBBE3. & Willing to do extra works which $\mathrm{b}$ & 1 & 2 & 3 & 4 & 5 & 6 & 7 \\
\hline
\end{tabular}


Organizational Commitment 216

\begin{tabular}{|c|c|c|c|c|c|c|c|c|}
\hline & responsibility & & & & & & & \\
\hline HBBE4. & $\begin{array}{l}\text { Willing to take extra responsibility to deal with new } \\
\text { organizational challenges }\end{array}$ & 1 & 2 & 3 & 4 & 5 & 6 & 7 \\
\hline HBBE5. & Proactively seek a better way of doing the job & 1 & 2 & 3 & 4 & 5 & 6 & 7 \\
\hline HBBE6. & Take your own risk in initiating new ways of doing things & 1 & 2 & 3 & 4 & 5 & 6 & 7 \\
\hline HBBE7. & $\begin{array}{l}\text { Support new ideas that you believe will bring comparative } \\
\text { advantages to the organization }\end{array}$ & 1 & 2 & 3 & 4 & 5 & 6 & 7 \\
\hline HBBE8. & $\begin{array}{l}\text { Actively monitor the work environment to respond quickly } \\
\text { to changes }\end{array}$ & 1 & 2 & 3 & 4 & 5 & 6 & 7 \\
\hline
\end{tabular}


WAVE 2: SUPERVISORS

Considerate Voice

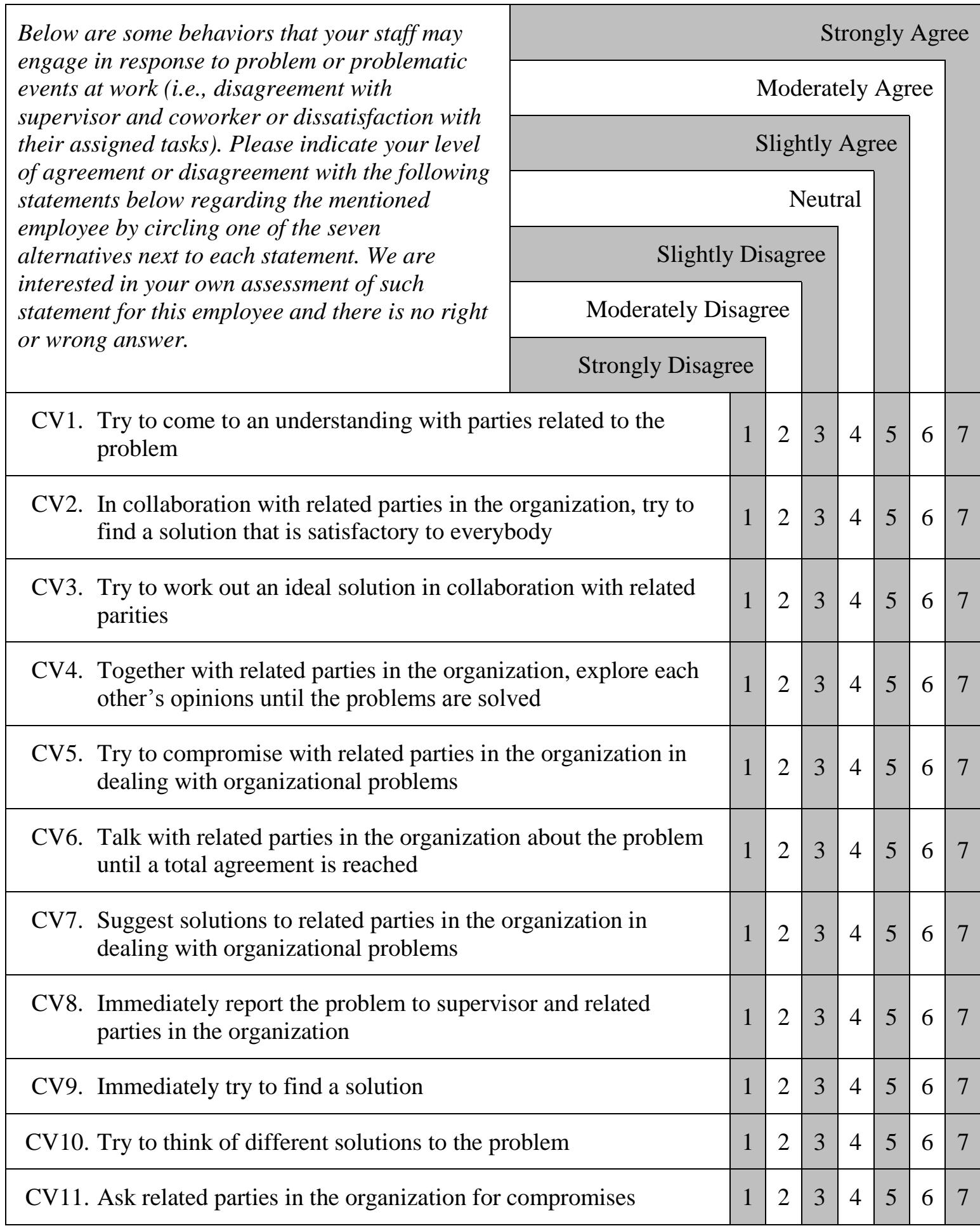


Production Deviance

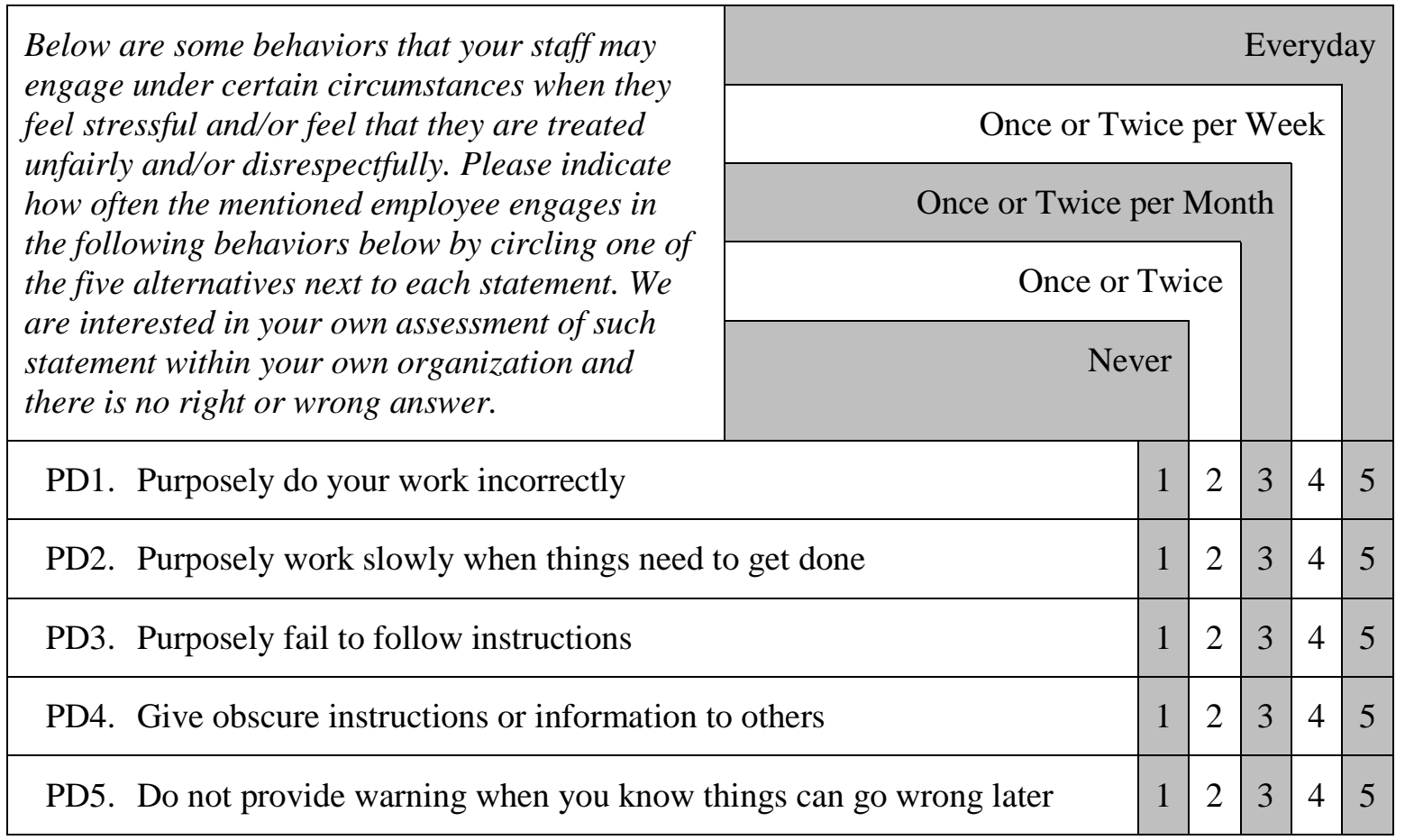

Behavior Engagement

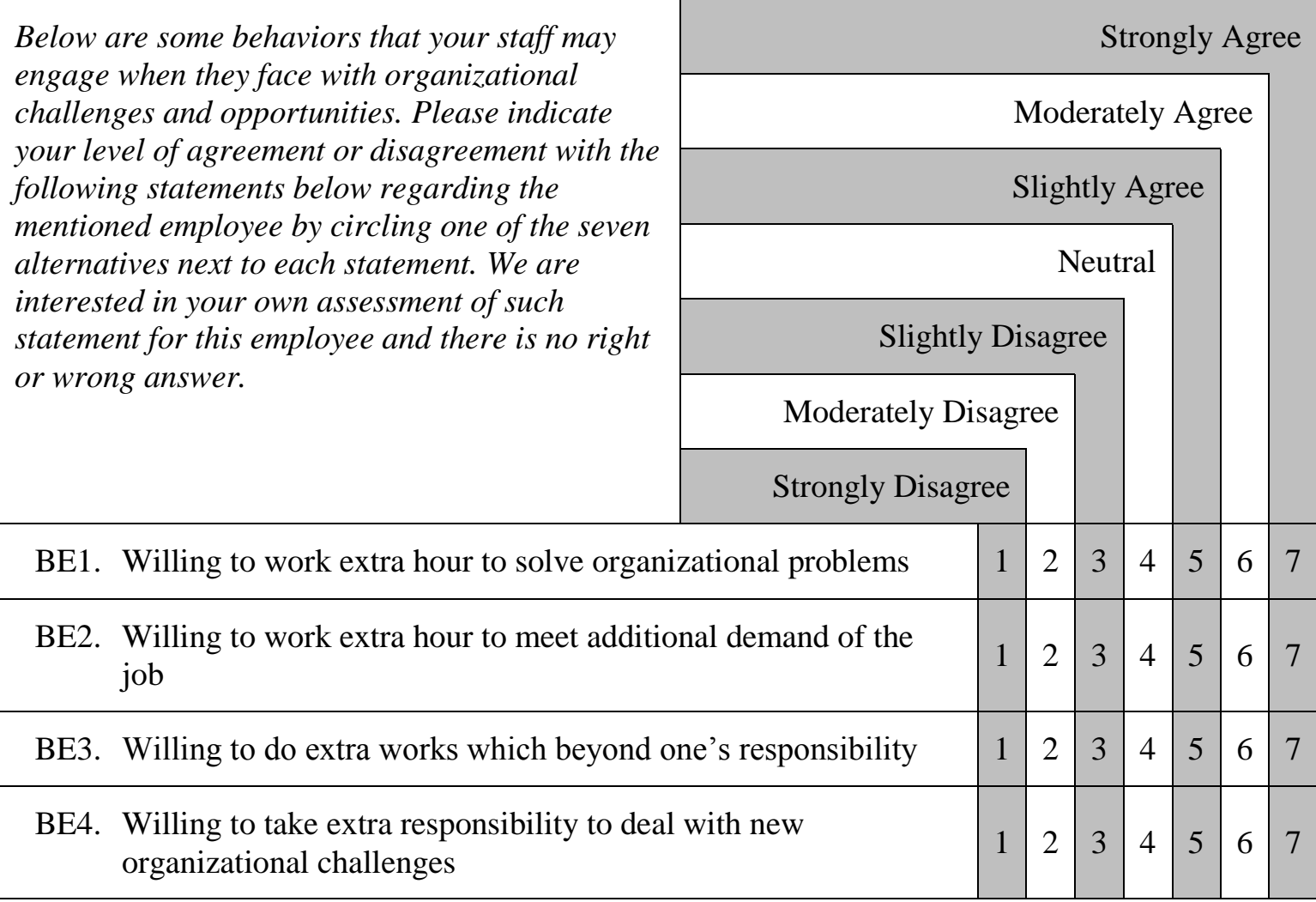


Organizational Commitment 219

\begin{tabular}{|c|c|c|c|c|c|c|c|}
\hline BE5. Proactively seek a better way of doing the job & 1 & 2 & 3 & 4 & 5 & 6 & 7 \\
\hline BE6. Take your own risk in initiating new ways of doing things & 1 & 2 & 3 & 4 & 5 & 6 & 7 \\
\hline $\begin{array}{l}\text { BE7. Support new ideas that you believe will bring comparative } \\
\text { advantages to the organization }\end{array}$ & 1 & 2 & 3 & 4 & 5 & 6 & 7 \\
\hline $\begin{array}{l}\text { BE8. Actively monitor the work environment to respond quickly to } \\
\text { changes }\end{array}$ & 1 & 2 & 3 & 4 & 5 & 6 & 7 \\
\hline
\end{tabular}

